Portland State University

PDXScholar

$5-15-2020$

\title{
Investigating Sexual Fantasy and Sexual Behavior in Adolescent Offenders
}

Hayley Lauren Tews

Portland State University

Follow this and additional works at: https://pdxscholar.library.pdx.edu/open_access_etds

Part of the Psychology Commons

Let us know how access to this document benefits you.

\section{Recommended Citation}

Tews, Hayley Lauren, "Investigating Sexual Fantasy and Sexual Behavior in Adolescent Offenders" (2020). Dissertations and Theses. Paper 5469.

https://doi.org/10.15760/etd.7341

This Dissertation is brought to you for free and open access. It has been accepted for inclusion in Dissertations and Theses by an authorized administrator of PDXScholar. Please contact us if we can make this document more accessible: pdxscholar@pdx.edu. 
Investigating Sexual Fantasy and Sexual Behavior in Adolescent Offenders

by

Hayley Lauren Tews

A dissertation submitted in partial fulfillment of the requirements for the degree of

Doctor of Philosophy

in

Applied Psychology

\author{
Dissertation Committee: \\ Keith Kaufman, Chair \\ Andrew Mashburn \\ Greg Townley \\ Kris Henning \\ Shannon Myrick
}

Portland State University

2020 


\begin{abstract}
Juvenile sexual offenders (SOs) have been found to account for a significant proportion of sexual offenses. A critical gap has been identified in our knowledge regarding the role of sexual fantasy and sexual behavior in juvenile sexual offending, even though these variables have been identified as an important treatment target for this population. Specifically, it is not clear if SOs differ significantly from juvenile non-sexual offenders (NSOs) in their sexual fantasies and sexual behavior and as such, if reports of fantasies and past sexual behaviors should be used as a target for sex offender specific treatment. This study explored differences on these two critical dimensions in a sample of 268 adjudicated, male, juvenile SOs and NSOs, in the care of the Oregon Youth Authority. Participants resided in four youth correctional facilities across the state of Oregon. The goals of this study were to, first, investigate differences in sexual fantasies experienced in the last 12 months by these subgroups. Second, to explore the predictive utility of deviant sexual fantasies and sexual behavior in predicting group membership (i.e., SO or NSO). Third, this study investigated differences in the relationship between deviant sexual fantasy and deviant sexual behavior for these two offender groups (i.e., SO and NSO). Finally, subgroup differences between these two groups' use of "sexting" were explored. Solitary sexual acts and voyeurism were significant predictors of offender group membership, after controlling for nondeviant sexual behavior, and significant group differences were also found in consensual sexting behavior. Overall, analyses focused on sexual fantasy resulted in nonsignificant results. Findings are discussed in relation to implications for treatment, prevention, and policy work in this area.
\end{abstract}




\section{Acknowledgements}

Completing this study would not have been possible without the guidance and support of the people in my corner. First and foremost, I would like to thank my advisor, Dr. Keith Kaufman, for his support and guidance throughout this process. Dr. Shannon Myrick, my supervisor at the Oregon Youth Authority and committee member, who gave me the flexibility and encouragement to make balancing a full-time job and progress on this dissertation, a possibility. Finally, Dr. Andy Mashburn, Dr. Greg Townley, and Dr. Kris Henning participated as committee members and contributed their expertise and support to me and this process.

Mom, Dad, and Riley, thank you for being my biggest cheerleaders! Alex, Emma, and Addy, thank you for your patience while I worked on this project and for encouraging me to keep on keeping on. I love you all! 
Table of Contents

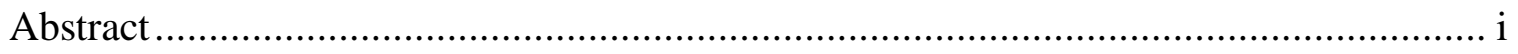

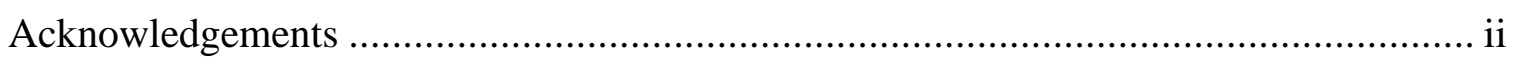

List of Tables................................................................................................... vi

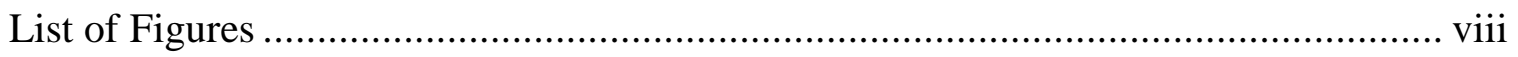

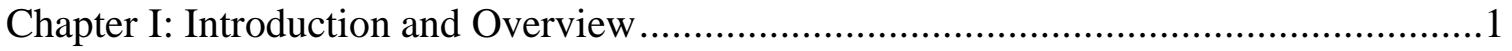

Defining Child Sexual Abuse .............................................................................2

Incidence of Child Sexual Abuse ........................................................................ 3

The Current Study ........................................................................................6

Chapter II: Juvenile Sexual Offenders.........................................................

Differentiating Juvenile and Adult Sexual Offenders ........................................... 10

Brain, cognitive, and psychosocial development in adolescence ......................... 12

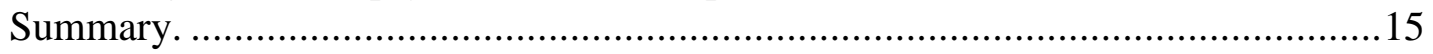

Differentiating Juvenile Sexual and Nonsexual Offenders ..................................16

Comparing Juvenile Sexual Offenders and Non-sexual Offenders..........................18

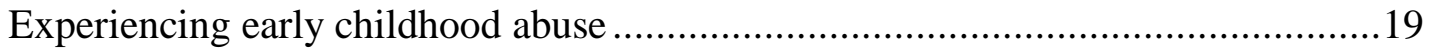

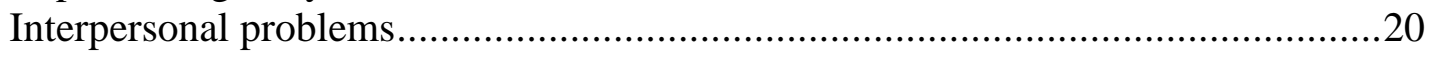

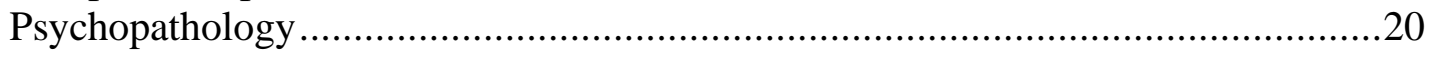

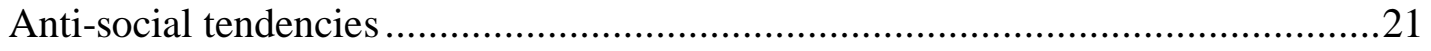

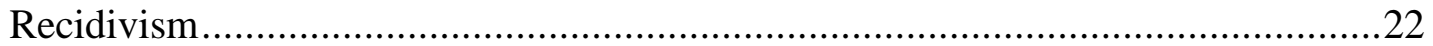

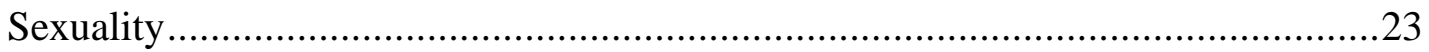

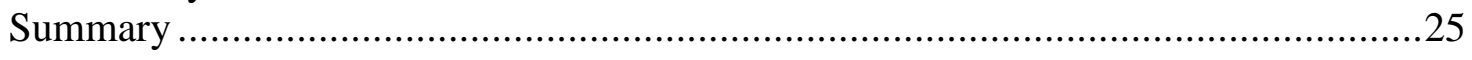

Chapter III: Theories of Sexual Offending .............................................................26

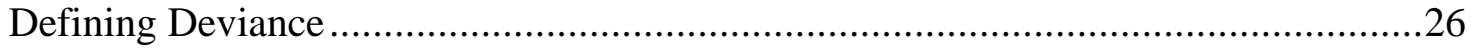

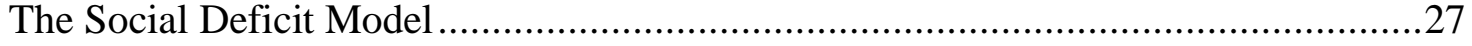

Conditioning Theory of Deviant Sexual Preference and Behavior ...........................30

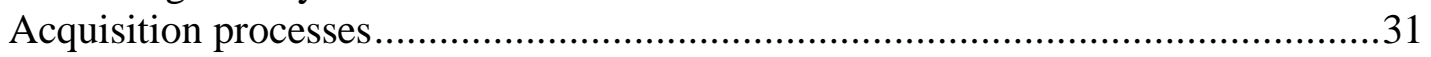

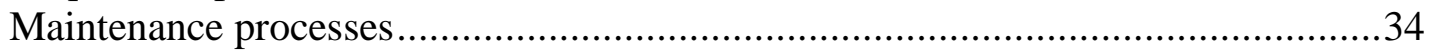

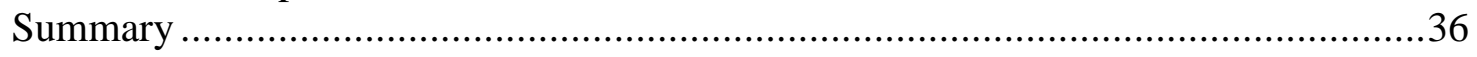

Chapter IV: Sexual Behavior of Juvenile Sexual Offenders ...................................38

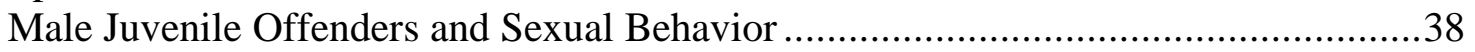

Advances in Technology and Sexting .............................................................40

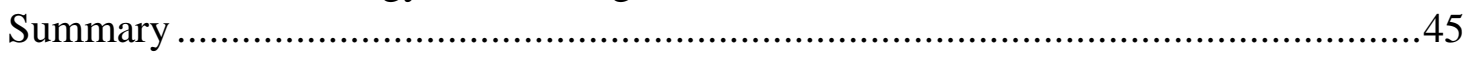

Chapter V: Sexual Fantasies of Juvenile Sexual Offenders......................................46

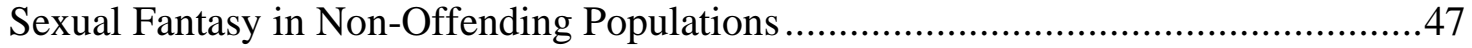

Sexual Fantasy in Sexual Offending...............................................................48

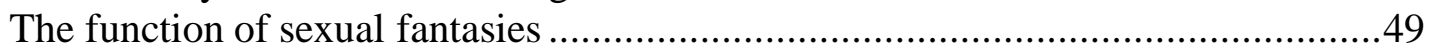

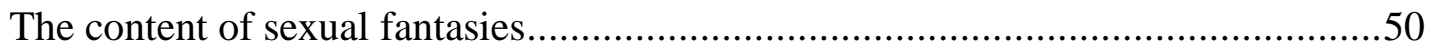

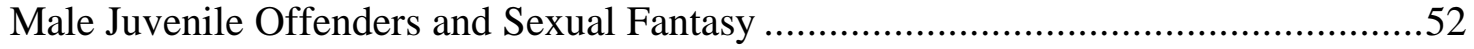

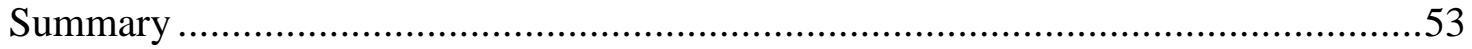


Chapter VI: The Role of Sexual Fantasy and Sexual Behavior in Prevention and Treatment ..................................................................................................... 55

The Significance of Sexual Fantasy and Sexual Behavior to Prevention Efforts .........55

The Significance of Sexual Fantasy and Sexual Behavior to Treatment Efforts ..........56

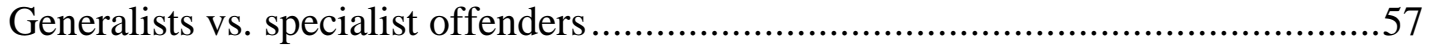

Sexual behavior as a treatment target .........................................................58

Sexual fantasy as a treatment target...............................................................59

Treating Deviant Sexual Arousal ........................................................................61

Critiques of Sexual Fantasy and Sexual Behavior as Treatment Targets ...................62

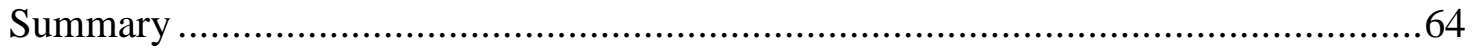

Chapter VII: Critiques of the Current Literature and Purpose of the Present Study ........65

Critiques of the Existing Literature on Juvenile Offenders ...................................65

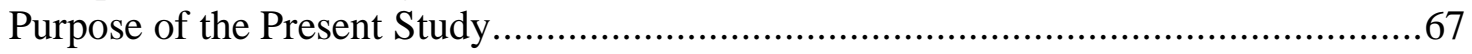

Chapter VIII: Development of Research Hypotheses ..............................................69

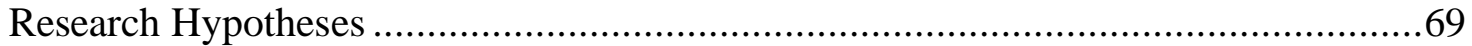

Subgroup differences in deviant sexual fantasies within the last year ...................69

Using sexual fantasy to predict group membership.......................................... 70

Using sexual behavior to predict group membership .................................... 71

Potential moderating effects of offender group on the relationship between juvenile

offenders' sexual fantasies and sexual behaviors ............................................. 72

The use of sexting by juvenile offenders ................................................ 74

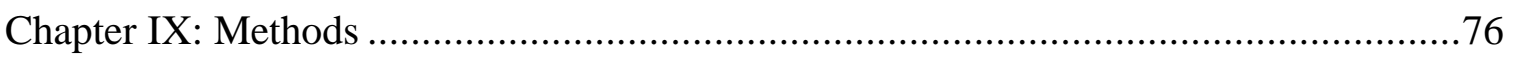

Institutional Review Board Process............................................................... 76

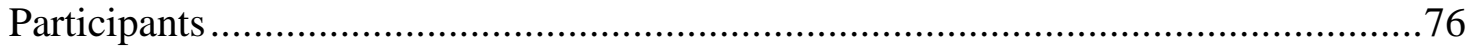

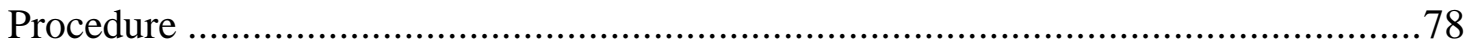

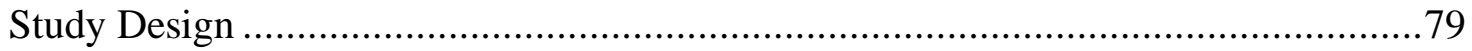

Descriptions and Measurement of Study Constructs .......................................... 79

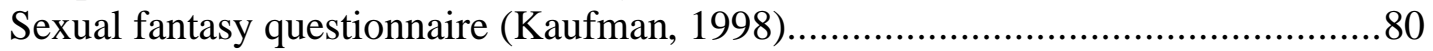

Sexual history form-revised (Kaufman, 2014) ............................................. 80

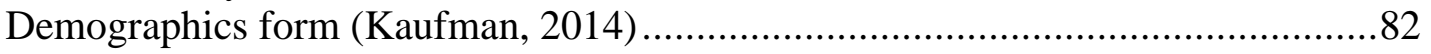

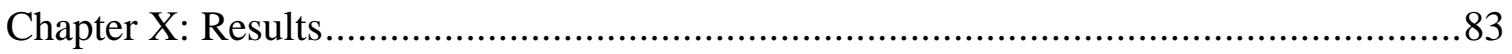

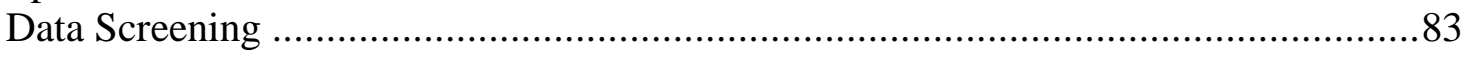

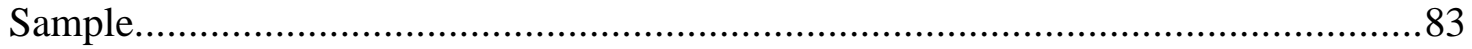

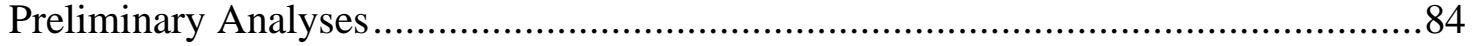

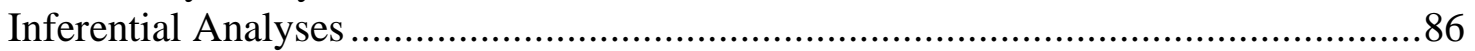

Subgroup difference in deviant 12-month sexual fantasies. ...............................86

Using sexual fantasy to predict offender group membership.............................8

Using sexual behavior to predict offender group membership. ...........................90

Potential moderating effects of offender group on the relationship between sexual fantasy and sexual behavior. ................................................................93

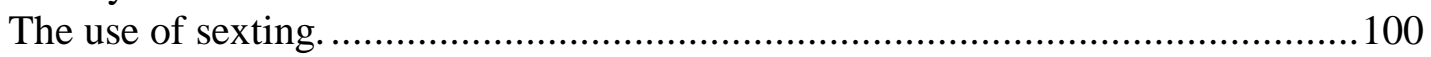

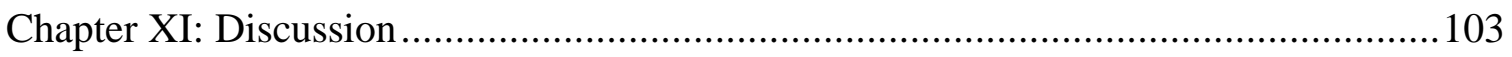




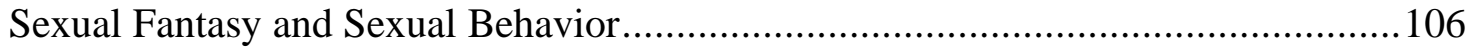

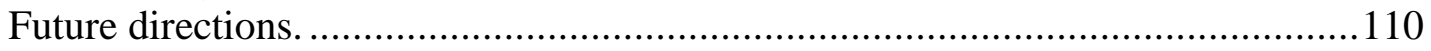

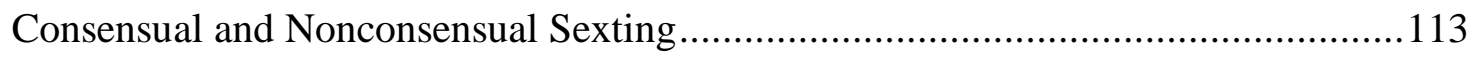

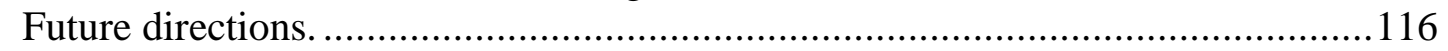

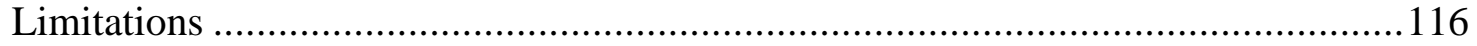

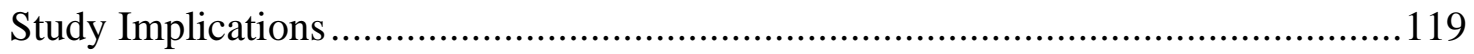

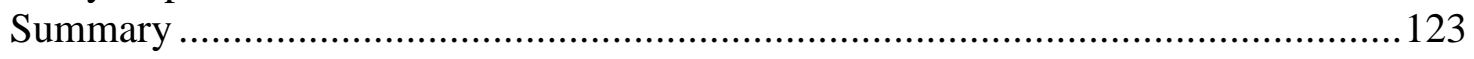

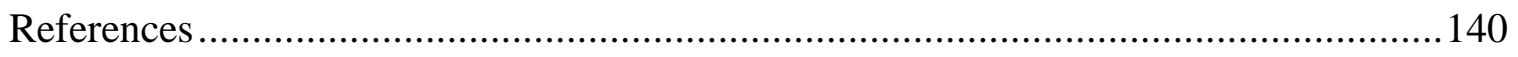


List of Tables

Table 1.

Sexual Fantasy Subscales

Table 2.

Sexual History Subscales

Table 3.

Correlation Matrix - Determining Covariates

Table 4.

Subscale Cronbach's Alpha Coefficients

Table 5.

Subscale Means and Standard Deviations

Table 6.

Hypothesis 2a: Comparison of Log-Likelihood Ratios

Table 7.

Hypothesis 2b: Comparison of Log-Likelihood Ratios

Table 8.

Hypothesis 3: Comparison of Log-Likelihood Ratios

Table 9.

Logistic Regression Predicting Group Membership Based on Social

Desirability, Nondeviant Sexual Behavior, and Deviant Sexual Behavior

Table 10.

Correlation Matrix - Consensual Sexting Items 
$\begin{array}{lr}\text { Table } 11 . & 136\end{array}$

\section{Means and Standard Deviations - Consensual Sexting Items}

Table 12.

Item Frequencies - Nonconsensual Sexting Items

Table 13.

Correlation Matrix Nonconsensual Sexting Items 
List of Figures

Figure 1.

Moderation of the Relationship Between Deviant 12-Month Fantasies and Aggressive Consensual Sexual Behavior by Offender Group (i.e., SO vs. NSO) 
Chapter I: Introduction and Overview

Child sexual abuse (CSA) has been identified as a national health problem and has resulted in the harm of millions of children (Murray et al., 2014). Due to its farreaching impacts on victims ${ }^{1}$, families of victims and offenders, and the communities they belong to, CSA has been identified by The Centers for Disease Control and Prevention and the World Health Assembly as one form of violence that puts public health at the greatest risk (McMahon \& Puett, 1999). While it is likely that prevalence estimates of the number of children affected by CSA are significantly underreported, it has been suggested that approximately 150 million girls and 73 million boys have been victims of contact CSA worldwide (e.g., penetration, fondling; Pinheiro, 2006). These numbers increase exponentially as the definition of what constitutes CSA is expanded to include non-contact offenses (e.g., voyeurism, exposure of the genitals). A common misconception of CSA is that it is a rare phenomenon that is perpetrated against females by unfamiliar, adult men (Murray et al., 2014). However, CSA occurs far too often, to males and females, and is most commonly (approximately $90 \%$ of cases) perpetrated by someone familiar to the victim (Murray et al., 2014; Snyder, 2000; Tofte \& Fellner, 2007). The sexual offender (SO) sample in this study is comprised primarily of offenders whose first victim was under the age of 18 . Of the participants who reported the age of their first victim, approximately $99 \%$ reported a victim under the age of 18 . Therefore, CSA is discussed in this first chapter, to understand the impact of most offenders

\footnotetext{
${ }^{1}$ The term "victim" is used throughout this document because this is the term most often used to describe those who have been subjected to CSA. It is acknowledged that the use of this term as opposed to survivor is a sensitive topic.
} 
included in this sample. In this chapter, CSA will be defined, followed by a discussion of prevalence rates, and the consequences experienced by victims.

\section{Defining Child Sexual Abuse}

Research has struggled with the development of a standardized definition of CSA. Variability in definitions of CSA across research studies and legal jurisdictions has made the comparison of systematic research and the development of a comprehensive body of research difficult; thus, stunting the forward growth of the field (Rowen, 2006). Even the subtlest differences, such as the acts included or the terminology used in the definition, can influence data collected on reporting efforts and the development of policy or legal response (Murray et al., 2014). In general, definitions, from both research and the legal fields, require two elements for an act to be labeled as CSA: (1) involving a child in sexual activities and (2) the presence of an "abusive condition" which may involve coercion that implies that the act was not consensual or that the victim was too young to give consent (Finkelhor, 1994). In an effort to capture CSA in a single, broad definition, The World Health Organization (WHO) defines CSA as:

"...the involvement of a child in sexual activity that he or she does not fully comprehend, is unable to give informed consent to, or for which the child is not developmentally prepared, or else that violates the laws or social taboos of society. Children can be sexually abused by adults or other children who are - by virtue of their age or stage of development - in a position of responsibility, trust or power over the victim (WHO, 2006 p. 10)."

This definition turns the focus to the relationship between the victim and the offender by spotlighting the role of power differentials. It also allows the inclusion of a range of acts including contact offenses (e.g., rape, touching without penetration) and noncontact 
offenses (e.g., voyeurism, taking sexual photographs of a child; Murray et al., 2014). Considering the challenges of developing a standardized definition of CSA, significant research efforts have taken place to most accurately represent the national incidence of CSA in the United States.

\section{Incidence of Child Sexual Abuse}

The number of children affected by CSA indicates that this is a significant national problem. The Fourth National Incidence Study (NIS-4; Sedlak et al., 2010) is a congressionally mandated research effort to determine the incidence of child abuse and maltreatment in the United States (US Department of Health and Human Services, nd). The National Incidence Studies have been completed once each decade since 1974 and measures the rates of child abuse and maltreatment by investigating cases that were both formally reported and those that were not (US Department of Health and Human Services, nd). Data for the NIS-4 were collected from a nationally represented sample of child protective service and sentinel agencies from 126 counties between September 2005 and May 2006. Results indicated that between 135,300 and 180,500 (22-24\%) children from this sample experienced some form of CSA with a vast majority (87\%) of the offenders being male.

It is important to note that data from the NIS-4 revealed a decline in the number of children who had experienced CSA when compared to the data collected for the NIS-3 in 1993 (Sedlak and Broadhurst, 1996). While this represents a 38-40\% decline in the number of cases of CSA, it is important to recognize that several factors may be influencing this apparent decline. For example, Finkelhor and Jones (2006) suggested 
that the decline may be due to fewer cases being reported and substantiated in more recent years. Reporting may be influenced by several factors. For example, younger children may lack the skills and resources necessary to communicate abuse that has occurred (Johnson, 2004; Taylor-Browne, 1997), if they are able to recognize CSA at all (Johnson, 2004). However, Finkelhor and Jones (2006) have concluded that at least some of the reported decline is likely due to an actual decrease in the number of incidences of CSA. This conclusion is supported by findings that reports of all types of child welfare (e.g., teen suicide, running away, juvenile delinquency) have declined and a decline in CSA has been found across all forms of reporting and all forms of sexual abuse (Finkelhor and Jones, 2006). Although CSA may be on the decline, it remains a significant problem worldwide and can cause significant distress to the victims that experience it.

\section{Consequences of Child Sexual Abuse}

The consequences of CSA experienced by each victim vary significantly depending on several factors. For example, consequences of CSA may be affected by the child's age at the onset of the abuse (Beitchman et al., 1992; Johnson, 2004; Paolucci, Genuis \& Violato, 2001), the child's sex (Beitchman et al.,1992; Paolucci et al., 2001), the child's development (Johnson, 2004), the physical acts performed during the abuse (Beitchman et. al.,1992), threats, bribes and force used during the abuse (Beitchman et. al.,1992; Johnson, 2004; Paolucci et al., 2001), fear of retribution (Johnson, 2004), fear of culpability (Johnson, 2004), frequency and duration of the abuse (Beitchman et. al.,1992; Johnson, 2004), the child's resilience (Johnson, 2004), family functioning (Beitchman et. 
al.,1992) and relationship to the perpetrator (Johnson, 2004; Paolucci et al., 2001). The individual characteristics of each victim will determine the constellation of consequences experienced.

Child sexual abuse can affect many areas of the victim's life and may include short-term, initial effects as well as long-term consequences. Short-term effects may include: emotional disturbance (Browne \& Finkelhor, 1986), fear (Browne \& Finkelhor, 1986), anxiety and hostility (Browne \& Finkelhor, 1986), and inappropriate sexual behavior (e.g., sexually “acting out”; Browne \& Finkelhor, 1986). Long-term effects can last well into adulthood and may include: depression (Beitchman et. al.,1992; Browne \& Finkelhor, 1986; Paolucci et al., 2001), negative effects on academic performance (Paolucci et al., 2001), self-destructive behavior (Browne \& Finkelhor, 1986; Paolucci, Genuis \& Violato, 2001), anxiety (Beitchman et. al.,1992; Browne \& Finkelhor, 1986), fear (Beitchman et. al.,1992), feelings of isolation and stigma (Browne \& Finkelhor, 1986), poor self-esteem (Browne \& Finkelhor, 1986), tendency toward revictimization (Beitchman et. al.,1992; Browne \& Finkelhor, 1986), substance abuse (Browne \& Finkelhor, 1986), posttraumatic stress disorder (PTSD; Paolucci et al., 2001; Rowan, 2006) and adult sexual dysfunction (Beitchman et. al.,1992; Paolucci et al., 2001; Rowan, 2006). In addition to the consequences that impact the victims, individually, the impacts of CSA reach far beyond the victim and the offender.

Child sexual abuse has significant impacts on the families of both the offenders and the victims (Wurtele, 2009). When abuse occurs within the family or at the hands of someone with a close relationship to the family, families can be torn apart through the 
process of investigation when they must decide who to believe and support (Jordan Institute for Families, 2000; Washington Coalition of Sexual Assault Programs, 2015). Families may experience shame for not recognizing the abuse sooner or guilt for putting their loved ones in a compromising situation (Jordan Institute for Families, 2000).

Finally, CSA has a significant economic impact on the larger community. The best available data on the cost of CSA comes from the Department of Justice who, in 1996, reported that child sexual abuse costs the United States approximately $\$ 1.5$ billion in medical expenses and $\$ 23$ billion dollars total annually (Miller et al., 1996; Wurtele, 2009). These expenses can be in the form of healthcare, criminal justice, child welfare, or special education costs (Fang et al., 2012). Additionally, productivity loss affects the community over time (Fang et al., 2012). With CSA being recognized as a national health problem, there is a significant amount of information that remains unknown about this phenomenon. The next section will outline the current study and identify how this project will begin to address a gap in current literature.

\section{The Current Study}

As an identified national health problem, effective prevention and treatment efforts are critical to reducing the incidence of CSA. In order to create effective programming and treatment planning, and reduce the negative impacts of CSA, it is important to understand the etiological risk factors of sexual offending behavior and the most effective targets for reducing the likelihood of future offenses. One way to do this is to study offender's behavior and thought patterns related to sexual offending. For decades, prevention and treatment efforts have focused on the role of sexual fantasy and 
sexual behavior in sexual offending (Abel \& Blanchard, 1974). The theoretical literature supports these constructs; however, the research literature has produced mixed findings regarding the role of sexual fantasy and sexual behavior in sexual offending behavior.

The purpose of the current study is to build upon the limited research available focusing on the role of sexual fantasy and sexual behavior in juvenile sexual offending. This study explored differences between juvenile SOs' and NSOs' sexual fantasies and sexual behavior. First, this study examined differences in the sexual fantasies and sexual behavior between juvenile SOs and NSOs. Second, differences in the engagement of sexting behavior between these two groups was investigated. To do this, a crosssectional, non-experimental design was utilized. Self-report survey data on sexual fantasy and sexual behavior was collected from incarcerated youth residing in youth correctional facilities, under the care of the Oregon Youth Authority.

Each of the following chapters will build a framework for this study by, first, distinguishing juvenile SOs as a subgroup of sexual offenders (Chapter 2) with unique characteristics and offense patterns. Chapter 3 will discuss theories that describe the development of sexual fantasy and sexual behavior for juvenile SOs. Chapter 4 will describe the role of sexual behavior in juvenile sexual offending followed by the role of sexual fantasy in juvenile sexual offending in chapter 5. Chapter 6 will discuss the current orientation of prevention and treatment efforts and the importance of sexual fantasy and sexual behavior in these efforts. Chapter 7 will provide a summarized critique of the current literature and identify how the current study planned to address these critiques. 
Following this introduction, Chapter 8 will outline the development of each research hypothesis explored in the current study. Development of research hypotheses will draw from previous research and theoretical literature for justification. Chapter 9 will discuss the methodology used for this study, including the intended participant groups, the measures used for data collection, and the procedures that will be used to collect the data. Chapter 10 will provide detailed results found relevant to each hypothesis. Finally, chapter 11 will conclude this dissertation with a discussion of the results, the limitations of the current study, and the implications of the findings. 


\section{Chapter II: Juvenile Sexual Offenders}

Many common perceptions of child sexual abuse (CSA) offenders are not supported by research findings and CSA offenders do not comprise a homogenous population. The stereotypical depiction of a CSA offender tends to be an adult male who is characterized as a pedophile (i.e., a sexual offender with primary sexual arousal to prepubescent children; Finkelhor et al., 2009). Contrary to this stereotype, research has suggested that juvenile sexual offenders ( $\mathrm{SO}$ ) make up a significant portion of sexual offenses and suggests that they should be treated as a unique subgroup (Kaufman et al., 1996; Kaufman et al., 1998). For example, of the known cases brought to the attention of the criminal justice system, data indicates that youthful offenders (i.e., offenders under the age of 18) are responsible for approximately $20 \%$ of rapes (Veneziano \& Veneziano, 2002). Additionally, juvenile offenders account for over one-quarter (25.8\%) of all SOs and more than one-third (35.6\%) of sexual offenses involving juvenile victims. Finally, estimates suggest that approximately $5 \%$ of juvenile SOs are younger than 9 years of age (Snyder \& Sickmund, 1999; Finkelhor et al., 2009).

An important distinction that is often overlooked in research on CSA is the difference between the behaviors and treatment needs of juvenile and adult SOs. Although research has identified critical differences in many characteristics between these two groups (e.g., offense characteristics, modus operandi), it is common for research findings that have been conducted exclusively on adult SO samples, to be applied to juvenile SOs. These significant differences highlight the field's need to better 
understand juvenile SOs to increase the effectiveness of their prevention and treatment efforts in the future (Pullman \& Seto, 2012).

This chapter will begin by providing support for treating juvenile SOs as a unique subgroup of sexual offenders. Next, a discussion of the ways in which adolescent development impacts behavior further supports the idea of treating juvenile offenders differently than adult offenders. Explanations of juvenile sexual offending will be described to provide a framework for the importance of exploring constructs of interest that differentiate juvenile SOs from juvenile non-sexual offenders (NSO). Research that has focused on identifying these constructs of interest and testing differences between these two groups of offenders will then be presented. Finally, a discussion of the harsh response to juvenile SOs by the justice system emphasizes the importance of understanding the etiology of this subpopulation of juvenile offenders.

\section{Differentiating Juvenile and Adult Sexual Offenders}

Several studies have sought to identify the unique characteristics of juvenile SOs, and several differences between juvenile and adult SOs have been found. For example, research has shown that juvenile SOs follow a unique offense pattern when compared to their adult counterparts. One difference is that juveniles are more likely to offend in groups of three or more perpetrators (Finkelhor et al., 2009). Additionally, juvenile SOs are more likely to offend against an acquaintance than a family member; whereas adults are more likely to offend against a family member than an acquaintance (Finkelhor et al., 2009). 
Important differences have also been found between the modus operandi of juvenile and adult SOs. The modus operandi of CSA refers to the "pattern of behaviors a perpetrator displays in the period prior to, during, and following illicit sexual contact" (Kaufman et al., 1996, p.18), or "the actions taken by an offender to perpetrate the offense successfully" (Douglas et al., 1997 p. 353 as cited in LeClerc et al., 2009). Kaufman and his colleagues (1998) compared the modus operandi of 114 juveniles and 114 adult SOs residing in correctional facilities using the Modus Operandi Questionnaire (MOQ; Kaufman, 1994). The MOQ asks participants to report the frequency with which they used a variety of strategies as part of their offending behaviors (Kaufman, 1994). Results indicated that juveniles used bribes and enticements to gain victim compliance (e.g., defending them when they were being bullied by other children, having a pet they wanted to see or play with), threats to involve the victim in sexually abusive acts (e.g., saying that you would hurt one of their family members if they did not come along, getting angry or violent with them), and manipulation to maintain victim silence (e.g., saying that their parent[s] wouldn't love them anymore because of the sexual activity, hoping they wouldn't want to lose you because you gave them so much attention) more often than adults (Kaufman et al., 1998). Effect sizes were not reported for this body of work, but subscale means were low, overall, for both juveniles and adults.

In a study comparing the perpetration characteristics of juvenile and adult SOs, Miranda and Corcoran (2000) used police reports, clinical notes, and in-person interviews to investigate differences. Participants were 16 juvenile and 19 adult male SOs who were currently living in the community, but participating in outpatient individual or family 
psychotherapy. Study results revealed that juveniles were more likely to utilize force during their abusive acts and to engage in "digital fondling (i.e., the use of fingers in sexual activity; p. 184)." Juveniles were less likely than their adult counterparts to engage in vaginal, anal, or oral intercourse.

Research investigating differences between juvenile and adult SOs has found significant differences in offense pattern, modus operandi, and characteristics of the abuse committed by these two groups. Recent advances in adolescent development also provides evidence for why juvenile offenders are significantly different than adult offenders. The next section provides an overview of adolescent development related juvenile offending.

Brain, cognitive, and psychosocial development in adolescence. In addition to significant differences between juvenile and adults in the literature specific to sexual offending, recent advances in the area of general adolescent development describes significant differences between juveniles and adults (Cauffman \& Steinberg, 2012). Specifically, adolescents are undergoing brain, cognitive, and psychosocial development that has a significant impact on their behavior and their decision-making.

Cauffman and Steinberg (2012) outline four significant ways that the brain develops during adolescence. First, in early adolescence, major changes occur in the brain's chemistry. Relevant to this discussion is the decrease in neurotransmitters dopamine and serotonin that occurs during this time and results in mood swings, difficulties regulating emotions, and decreased impulse control (Cauffman \& Steinberg, 2012). These changes are rapid and lead to increases in sensation seeking behavior 
(Cauffman \& Steinberg, 2012; Arain et al., 2013). Second, childhood and early adolescence is a period, during which, synaptic "pruning" is eliminating unused synapses and creating more efficient neural connections (Cauffman \& Steinberg, 2012). Through this process, primarily occurring in the prefrontal cortex, adolescents are experiencing improvement in executive functions (Cauffman \& Steinberg, 2012; Arain et al, 2013). Third, during this time, the process of myelination is insulating neural pathways, improving speed and reliability of signal transmission (Cauffman \& Steinberg, 2012; Arain et al, 2013). Myelination is an important process for supporting higher order functioning, such as, response inhibition, planning, cost-benefit analysis, and reconciling information from several sources (Cauffman \& Steinberg, 2012). Finally, into late adolescence is a period of continued growth of the neural connections between cortical and subcortical regions of the brain (Cauffman \& Steinberg, 2012). These connections contribute to improvement in emotional self-regulation, including judgements about risk and reward and cognitive control over emotional impulses (Cauffman \& Steinberg, 2012). When considering adolescent brain development, a significant gap exists that must be highlighted. The changes in the neurotransmitters, dopamine and serotonin, occur rapidly; whereas, the processes of synaptic "pruning," myelination, and neural connections between the cortical and subcortical regions of the brain are much slower (Cauffman \& Steinberg, 2012). Therefore, there is a significant gap between the onset of sensation seeking behaviors and the development of higher order, executive functioning.

Adolescence is also an important period for cognitive development. Capacity to understand and reason increases through childhood into adolescence. Specifically, 
between the ages of 11 and 16, adolescents' ability to reason deductively and process information improves (Hale, 1990; Kail, 1997; Keating, 2004; Overton, 1990 as cited in Cauffman \& Steinberg, 2012). These are both important to abstract and hypothetical thinking (Kuhn, 2009). In general, these abilities peak around the age of 16 and, at this point, are comparable to those of adults (Cauffman \& Steinberg, 2012). However, while at the age of about 16, adolescents can understand and reason, maturity of judgement is not comparable to adults (Cauffman \& Steinberg, 2012). Maturity of judgement is heavily reliant on psychosocial development, when emotional and social variables are impacting decision making (Cauffman \& Steinberg, 2102). With cognitive development, comes the development of moral reasoning. The development of morality begins with a respect for rules and authority, more focused on a fear of consequence (i.e., Pre-Moral; Kohlberg, 2008). In the intermediate stage, motivation is characterized by anticipation of praise or blame, more focused on maintaining relationships (i.e., Morality of Conventional Role-Conformity; Kohlberg, 2008). Whereas, the final stage of moral development is characterized by social perspective taking, where an individual moves beyond their own perspective and considers abstract principles and values and their impact on others (i.e., Postconventional level; Kohlberg, 2008). It becomes more about not wanting inflict harm on others.

Psychosocial development continues beyond mid-adolescence and into young adulthood. The areas of psychosocial development relevant to juvenile offending are peer influence, future orientation, reward sensitivity, and self-regulation (Cauffman \& Steinberg, 2012). Adolescents are more oriented towards peers and responsive to peer 
influence than adults (Steinberg \& Monahan, 2007). This manifests in young people taking risks they might otherwise avoid, in direct response to peer pressure (Cauffman \& Steinberg, 2012). In addition, adolescents' desire for approval, and alternatively, fear of rejection from their peers, affects the choices that they make (Cauffman \& Steinberg, 2012). A second domain that develops across adolescence is future orientation. Future orientation, or "the capacity and inclination to project events into the future," affects the extent to which individuals consider long-term consequences (Cauffman \& Steinberg, 2012, p. 435). Over the course of adolescence, individuals become more concerned about their future and see marked improvements in their ability to consider future consequences and plan ahead (Greene, 1986; Nurmi, 1991; Steinberg et al., 2009 as cited in Cauffman \& Steinberg, 2012). The third domain of psychosocial development relevant to this work is reward sensitivity. When faced with something desirable, adolescents are more likely to act (Gardner \& Steinberg, 2012). Research suggests that while adolescents are able to identify risk similarly to adults, adolescents tend to weigh the reward in risk taking more heavily than consequences (Gardner \& Steinberg, 2012, Steinberg, 2004). Finally, adolescents are experiencing steady increases in their capacity for self-direction and declines in impulsivity into young adulthood (Steinberg et al., 2008).

Summary. Research literature has identified significant differences between adults and juvenile SOs, specific to the perpetration of sexual offending. While this research literature is limited, we can turn to advances in our understanding of adolescent brain development, to further support the need to understand juvenile SOs as a distinct population of SOs. During adolescence, youth are developing significantly, in many 
ways. First, their brains are developing the appropriate connections to control their impulses, improve their executive functions, plan, and regulate their emotions.

Cognitively, they are developing maturity of judgement and hypothetical thinking.

Finally, psychosocially, they are developing skills related to resisting peer pressure and understanding the impacts of their behavior.

Adolescent development is a critical factor is determining why juveniles commit crime. While recent advances in adolescent development help to explain differences in why juveniles and adults commit crimes, it has been suggested that juvenile SOs also represent a unique subgroup of juvenile offenders who exhibit a different etiology than juvenile nonsexual offenders (NSO). In the next sections, a description of two underlying theories that seek to explain juvenile sexual offending will be followed by a review of the research literature discussing the differences between juvenile SOs and NSOs.

\section{Differentiating Juvenile Sexual and Nonsexual Offenders}

A highly debated topic in the field of juvenile sexual offending is whether juvenile SOs are "generalist" or "specialist" offenders. The generalist explanation of juvenile sexual offending states that sexual offending is simply a manifestation of general antisocial tendencies and criminal behavior (Pullman \& Seto, 2012; Seto \& Lalumiere, 2010). The generalist explanation suggests that there are more similarities than differences between juvenile SOs and NSOs (Pullman \& Seto, 2012). This would mean that these two groups share the same risk factors and could benefit from similar assessment and treatment efforts (Pullman \& Seto, 2012). Studies supporting the generalist explanation have found that juvenile SOs were similar to NSOs on constructs 
such as psychiatric history, past delinquency, parenting, and school experiences (Pullman \& Seto, 2012).

In contrast, a specialist explanation of juvenile sexual offending suggests that sexual offending has a unique set of etiological factors, such as sexual abuse history, atypical sexual experiences, and atypical sexual interests (Pullman \& Seto, 2012; Seto \& Lalumiere, 2010). A specialist explanation suggests that these two groups are more different than they are similar (Pullman \& Seto, 2012). The specialist perspective indicates that juvenile SOs and NSOs require different assessment tools and treatment approaches (Pullman \& Seto, 2012). Studies supporting the specialist explanation have found significant differences between juvenile SOs and NSOs on theoretically important constructs, such as: exposure to other's sexual behavior in their past, arousal to deviant stimuli, and victimization of child sexual abuse (Pullman \& Seto, 2012). A specialist perspective also holds that sexual offenders commit sexual offenses and engage in this type of behavior repeatedly and frequently (Harris et al., 2009).

Empirical findings on the generalist versus specialist explanations of juvenile sexual offending are mixed (Pullman \& Seto, 2012). Results are often influenced by the composition of juvenile SOs that make up a single study (e.g., close custody versus residential treatment facility, sample size, treatment curriculum offenders are engaged in). Pullman and Seto (2012) suggest that it is possible that the generalist and specialist explanations of juvenile sexual offending are not mutually exclusive. For example, it may be that juvenile SOs are similar to NSOs on some theoretically derived variables (e.g., 
antisocial tendencies) and different on others (e.g., sexuality). The next section will discuss research that has sought to examine differences between juvenile SOs and NSOs.

\section{Comparing Juvenile Sexual Offenders and Non-sexual Offenders}

Mixed evidence makes it difficult to definitively conclude whether juvenile SOs are specialists or generalists, further indicating the need for more research investigating differences between these two groups of offenders. While some authors conclude that juvenile SOs more closely resemble a normative population than delinquent populations (e.g., Fagan \& Wexler, 1988), Seto and Lalumiere (2010) conducted a meta-analytic review of relevant research and concluded that a generalist explanation of juvenile sexual offending is not sufficient. This section explores the research literature that currently exists comparing juvenile SOs and NSOs.

In a seminal meta-analytic review examining specialist explanations of juvenile SOs by comparing them to juvenile NSOs on theoretical constructs of interest, Seto and Lalumiere (2010) concluded that sexual offending could not be explained as a manifestation of general antisocial tendencies. A unique etiology of sexual offending behaviors in juveniles was supported by findings of differences on several theoretically important constructs. For example, although juvenile SOs tended to have extensive criminal histories and exhibited antisocial tendencies, they were more likely to score lower on measures of antisocial attitudes and beliefs, overall, than juvenile NSOs, but higher than non-offenders. Juvenile SOs also had fewer substance abuse issues, overall, when compared to juvenile NSOs. To ensure a comprehensive literature review, research published after 2010 (when Seto and Lalumiere [2010] published their meta-analytic 
review) will be discussed in addition to conclusions drawn from that review, as appropriate.

Experiencing early childhood abuse. Many constructs related to early childhood abuse did show significant differences between juvenile SOs and NSOs, supporting a specialist explanation of sexual offending. In Seto and Lalumiere's (2010) meta-analysis, a total of 34 studies were reviewed within this domain. First, juvenile SOs reported a more frequent history of experienced sexual abuse, overall, than their NSO counterparts (Seto \& Lalumiere, 2010). Effect sizes indicated that these differences were significant and "medium-to-large" (i.e., between .50 and .80) in size (Seto \& Lalumiere, 2010). Odds-ratios revealed that juvenile SOs had over five times (i.e., 5.35) greater odds than juvenile NSOs of experiencing CSA (Seto \& Lalumiere, 2010). Childhood physical abuse showed similar, but weaker, differences between these two groups (Seto \& Lalumiere, 2010). The effect sizes indicated that these differences were significant and "small" (i.e., between .20 and .50) in size (Seto \& Lalumiere, 2010). Overall, odds ratios revealed that juvenile SOs were 1.6 times more likely than their NSO counterparts to experience childhood physical abuse (Seto \& Lalumiere, 2010). Differences in the experience of physical abuse between these two groups were significantly different when the information collected was from self-report measures, but not for studies that collected information from other sources (Seto \& Lalumiere, 2010). Finally, experiences of neglect were more frequently reported by juvenile SOs than juvenile NSOs (Seto \& Lalumiere, 2010). The effect sizes, in this case, were "small to medium" (i.e., between .20 and .50) in size (Seto \& Lalumiere, 2010). 
Interpersonal problems. Seto and Lalumiere (2010) compared juvenile SOs and NSOs on four variables across 22 studies in the interpersonal problems domain: 1) heterosocial skills deficits; 2) general social skills deficits; 3) social isolation; and 4) "other social problems that can interfere with the development or maintenance of relationships with others." Of the four variables tested, only one reached statistical significance. Social isolation was more frequently reported by juvenile SOs than by NSOs. Effects sizes resulting from these differences were "small" (i.e., between .20 and .50), but significant. There were no significant differences between the groups on the other three variables in this domain (Seto \& Lalumiere, 2010).

Psychopathology. Seto and Lalumiere (2010) reviewed 23 studies comparing the two groups of interest on the domain of psychopathology. In addition to general psychopathology, Seto and Lalumiere (2010) examined differences in seven more specific domains related to psychopathology: 1) anxiety; 2) social anxiety; 3) depression; 4) neuroticism; 5) psychotic symptoms; 6) suicidal tendencies; and 7) low self-esteem. Juvenile SOs were more likely to report anxiety and low self-esteem than NSOs. Effect sizes indicated that these differences were "small" (i.e., between .20 and .50), but significant. Significant differences were not found for general psychopathology or the other five more narrow domains.

Cale and colleagues (2015) were interested in exploring differences in psychopathic personality disturbances between juvenile SOs and NSOs. In a sample of 263 incarcerated, male, juvenile offenders from British Columbia, participant scores were created using the Psychopathy Checklist: Youth Version (PCL: YV). This symptom 
rating scale is coded from semi-structured interviews lasting between 60-90 minutes, as well as information drawn from participant files. The PCL: YV contains 20 items on a 3point Likert-type scale from 0 (Item does not apply) to 2 (Item definitely applies). Information on the specific content of each of the items in each of these dimensions was not provided. The authors concluded that juvenile SOs, overall, had significantly higher total scores of psychopathy compared to juvenile NSOs. Specifically, SOs had more participants with "high" PCL: YV scores (i.e., above 25 out of 60$)$ than the NSOs $(32.5 \%$ vs $9.4 \%$, respectively). Further investigation indicated that this difference was primarily driven by juvenile SOs' scores being significantly higher on the interpersonal and emotional/affective dimensions of psychopathy than those of their non-sexual offending counterparts. Scores did not differ significantly between these two groups on the behavioral/lifestyle or antisocial domains.

Anti-social tendencies. Supporting the finding from Cale and colleagues (2015) and in contrast to Seto and Lalumiere's (2010) conclusion, McCuish and colleagues (2015) determined that there were more within-group differences in antisocial behavioral antecedents for both juvenile SOs and NSOs than between-group differences. McCuish and colleagues (2015) analyzed the interview transcripts and official file information of 145 incarcerated, male, juvenile offenders in British Columbia. Interviews contained the Measurement of Adolescent Social and Personal Adaptation in Quebec (MASPAQ), which measures authority conflict, covert behavior, and overt behavior. Using latent class analysis, results indicated three latent classes: low antisocial, overt, and covert. The low antisocial group had low item responses for items measuring antisocial tendencies. The 
overt group has the highest probability of having engaged in violent or aggressive behaviors (e.g., fist fights). Finally, the covert group had the highest probability of engaging in covert forms of behavior (e.g., theft). The two groups did not significantly differ on any of the latent class behavioral pathways. For both groups, most of the participants (approximately 50\%) fell into the "Low Antisocial" class. For both groups, the remainder of the sample was almost evenly split between the "Overt" and "Covert" groups. As a result, it was suggested that the classification of juvenile SOs as antisocial or non-antisocial may be too simplistic (McCuish et al., 2015).

Recidivism. Juvenile SOs and NSOs have also been found to differ on recidivism and related factors. Calleja (2013) compared 40 male, juvenile SOs to 130 male, juvenile NSOs from a residential treatment facility. Recidivism data was collected from a statewide database for a period of up to two years post-release from the facility. For this study, recidivism was defined as "a new criminal offense that resulted in disposition in either the juvenile or the adult criminal justice system (p 6; Calleja, 2013)." Based on the information contained in the statewide database, none of the SOs, in this sample, reoffended sexually within the two-year tracking period. Of the SOs in this sample, $3 \%$ reoffended with a non-sexual crime during the two-year tracking period. Overall, NSOs were between $5 \%$ and $8 \%$ more likely to reoffend within this tracking period. Sexual offenses did not represent any of the recidivism crimes (Calleja, 2013). Calleja (2013) did note that because juvenile SOs were classified as having "special needs", their treatment may have included a higher degree of services or resources as compared with NSOs. Differences may include increased supervision and provision of supports (Calleja, 2013). 
Sexuality. Seto and Lalumiere (2010) reported on the paucity of research comparing the sexuality of juvenile SOs and NSOs. For this meta-analysis, sexuality included sexual development, experience, and interest. Overall, juvenile SOs reported more exposure to sex and/or pornography, diagnoses of paraphilias, and atypical sexual fantasies. Specific studies focused on these constructs will be reviewed in a later chapter (see Chapters 4 and 5 for details).

\section{The Justice Systems Response to Juvenile Sexual Offenders}

Continuing to forward our understanding of the etiology of juvenile SOs is critically important because the penalties that these youth face in the US are more severe than in any other democratic nation (Letourneau \& Caldwell, 2013). Sensational media coverage in the late 1980s ignited fear of a "sex crime epidemic" and current policy is a lasting effect of the public's fear of that time (Letourneau \& Caldwell, 2013).

Post-incarceration civil commitment was established as a way to keep those who had been determined to be a "sexually violent predator" committed after offenders had completed their sentences imposed by the courts (Letourneau \& Caldwell, 2013). Prior to their release, offenders undergo evaluation to determine if they have mental deficiencies and are likely to commit violent sexual crimes in the future (Letourneau \& Caldwell, 2013). Commitment in these situations is indefinite and it is rare that someone under a post-incarceration civil commitment is released (Letourneau \& Caldwell, 2013). In many states, post-incarceration civil commitment applies to juvenile SOs (Letourneau \& Caldwell, 2013). 
In the mid 1990s, federal law required states to create sex offender registries and establish laws to notify the public of information on sex offenders (Letourneau \& Caldwell, 2013). In 2006, the Adam Walsh Act required juveniles to register for 25 years to life, depending on the nature of their crime and offense history (Human Rights Watch, 2013; Letourneau \& Caldwell, 2013). Lifetime registration assumes that juvenile sexual misconduct is a permanent issue for the individual, while evidence shows that it is incredibly responsive to treatment and maturation (Human Rights Watch, 2013;

Letourneau \& Caldwell, 2013).

While well intended, these policies rely on unreliable recidivism risk prediction (Letourneau \& Caldwell, 2013). Risk prediction for juveniles is complicated by several factors. First, the base rate for commission of subsequent sexual offenses by juveniles is low, making it a difficult outcome to target with intervention and prediction (Human Rights Watch, 2013; Letourneau \& Caldwell, 2013). Second, juvenile SOs are a heterogeneous population with a constellation of risk factors that varies by individual (Letourneau \& Caldwell, 2013). Finally, juvenile behavior is significantly impacted by the developmental changes that are occurring at this stage in adolescence and are likely to be unstable, making them difficult to predict (Letourneau \& Caldwell, 2013). Although fairly widely accepted, these policies have not been linked to their intended effect of reducing sexual offending (Human Rights Watch, 2013).

In addition to being ineffective in reducing sexual offending, these policies have significant long-term repercussion for juvenile SOs (Chaffin, 2008). Being labeled as "sex offenders" has been linked to stigma, isolation, shame, and depression (Human 
Rights Watch, 2013). Youth and their families have reported becoming the victims of significant physical violence (Letourneau \& Caldwell, 2013). For those impacted, stability has been elusive due to the necessity of frequent moves, being denied access to education, and being unable to maintain employment (Letourneau \& Caldwell, 2013).

\section{Summary}

Juvenile SOs have been identified as a unique subgroup of sexual offenders and have been suggested to be a unique subgroup of juvenile offenders. In a meta-analytic review testing the specialist explanation of juvenile sexual offending, Seto and Lalumiere (2010) concluded that juvenile SOs do exhibit several significant differences from their NSO counterparts. Seto and Lalumiere (2010) recommend that more research comparing these two groups be done to better understand juvenile sexual offending. More specifically, they highlighted the lack of research that exists around sexuality and its role in offending. The next chapter will discuss theories that describe the development of sexual fantasy and sexual behavior for juvenile SOs. 


\section{Chapter III: Theories of Sexual Offending}

As outlined above, juvenile sexual offenders (SOs) represent a heterogeneous group that engages in a wide variety of behaviors (Fagan \& Wexler, 1988; Rich, 2006). This heterogeneity has led the field to struggle in developing a single theory to explain the intricacies of juvenile sexual offending (Fagan \& Wexler, 1988). There are two primary theories of sexual offending that are relevant to the study of sexual fantasy and sexual behavior as etiological factors, the Social Deficit Model (Marshall et al., 1993) and the Conditioning Theory of Deviant Sexual Preference and Behavior (Laws \& Marshall, 1990). Both suggest that juvenile SOs are likely to engage in more deviant sexual behaviors and have more deviant sexual fantasies than juvenile non-sexual offenders (NSO). In this chapter, the difficulties in defining and using the term "deviant" to describe sexual behaviors will be discussed. After defining deviance, the social deficit model and conditioning theories will be described in more detail.

\section{Defining Deviance}

A link between deviant sexual fantasies and deviant sexual behavior has been widely acknowledged for quite some time (Aylwin et al., 2005). However, the use of the term "deviant" is problematic because what has traditionally been thought of as deviant fantasies have been found to also be present in non-offending populations (Bartels \& Gannon, 2011; Joyal et al., 2015; Leitenberg \& Henning, 1995). Traditionally, the word deviant refers to "behavior which contravenes accepted standards of behavior, often called social norms" (Larsen, 2013 p 1). While the concept of deviance appears to be universal across cultures, there is little agreement as to what activities and behaviors 
constitute deviance, even within a particular culture (Larsen, 2013). Social norms have a significant influence on what is determined to be deviant, yet social norms are often created by the majority and do not capture the values of an entire population (Larsen, 2013). Additionally, attitudes and conceptualizations of what is deemed deviant changes over time (Larsen, 2013). Sodomy was a capital crime as recently as 200 years ago (Larsen, 2013). Homosexuality was included in the Diagnostic and Statistical Manual of Mental Disorders (DSM) II, published in 1968, as a sexual deviation, but was removed in the DSM III, published in 1980, because it no longer fit with the definition of a mental disorder (De Block \& Adriaens, 2013).

The existing literature does not offer a formal definition for "deviant" sexual behavior and fantasies (Bartels \& Gannon, 2011). For the present study, deviance will be defined similarly to the limited available research in the field, following the definition given by Larsen (2013). It is important for the purposes of the present study, that the definitions of constructs of interest remain similar. If definitions are different across research studies, it is impossible to determine if the construct has changed over time or if the findings are the result of measuring something different. The next section will describe the social deficit model of sexual offending and its relevance to the current study.

\section{The Social Deficit Model}

The Social Deficit Model of sexual offending suggests that offenders choose inappropriate partners because they are unable to achieve intimacy or establish sexual relationships with developmentally appropriate partners (Daleidan et al., 1998; Marshall 
et al., 1993). It has been long understood that a common characteristic of juvenile SOs is poor social relations (Marshall et al., 1993). During the onset of puberty, adolescents begin seeking out the company of their peers more frequently and the company of their primary care givers less frequently (Marshall et al., 1993). For most adolescents, this transition can be challenging, as it is a period of personal discovery and of gaining acceptance from peers (Marshall et al., 1993). However, for children who have experienced an insecure attachment to their primary caregivers, this transition is even more difficult. Insecure attachment occurs when parents respond inconsistently to the needs of the child or when the primary caregiver is detached and unresponsive to the child's needs (Simons et al., 2008). Adolescents who have experienced insecure attachment to their primary caregivers have an intense fear of rejection and intimacy, tend to lack confidence in themselves, and are less likely to have developed the skills required to establish close relationships with same aged peers (Marshall et al., 1993). This muted ability to form close relationships with same aged peers is exacerbated when adolescents attempt to form relationships with female peers (Marshall et al., 1993).

Due to lagging skills and a fear of rejection and intimacy, these adolescents begin to meet their emotional needs through physical gratification (e.g., masturbation, aggressive behavior; Marshall et al., 1993). For juvenile SOs, this attempt at gratification manifests itself through the physical act of sex (Marshall et al., 1993). The act of forcing a peer to have sex or engaging in forced sexual activity with a young child does not require the socio-relational skills that these adolescents might be lacking, nor does it make them vulnerable to rejection or raise issues related to their lack of intimacy with 
peers (Marshall et al., 1993). Instead, it becomes about power, control, and achieving gratification.

It is also interesting to note that the sexual fantasies of adolescents with insecure attachment tend to follow non-affectionate themes (Marshall et al., 1993). The focus of the fantasy is often centered on the act itself, instead of the more romantic or intimate context within which the sexual act is occurring. Focusing the content of the fantasy on the act itself distances the adolescent from vulnerability to rejection and the lack of intimacy in their relationships (Marshall et al., 1993). For example, a fantasy may be focused on a young child performing fellatio on the adolescent as opposed to an emphasis on the larger context that would call attention to their lack of age-appropriate romantic relationships and their skills deficits in these areas. This narrow focus also obscures the coercion or force involved with getting the child to perform the sexual act. Masturbatory fantasies tend to involve compliant partners who succumb to the wishes of the individual having the fantasy (Marshall et al., 1993). This content allows the individual having the fantasy to avoid their anxiety related to rejection.

Theorists acknowledge that insecure attachment is an experience that can be shared by juvenile SOs and NSOs (Marshall et al., 1993). It follows, then, that juvenile SOs may not appear different than juvenile NSOs in the number or composition of peer attachments (Marshall et al., 1993). Instead, it has been suggested that the difference between these two groups is the extent to which juvenile SOs are preoccupied with sexual thoughts. Sexual preoccupation manifests as 'an abnormally intense interest in sex that dominates psychological functioning' (Mann et al., 2010, p. 198). Considering the 
significant development occurring in the adolescent brain that decreases impulse control and increases sensation seeking (Cauffman \& Steinberg, 2012), coupled with sexual preoccupation, juvenile SOs would be more likely to engage in sexual activity to meet their innate needs for intimacy and closeness.

Given this sexual preoccupation and their inability to form developmentally appropriate relationships, it would be expected that juvenile SOs would be more likely to have fewer "traditional" sexual experiences with appropriately aged, consenting partners than juvenile NSOs (Daleiden et al., 1998). The social deficits model helps to explain why juvenile SOs might seek out inappropriate/illegal child partners. In the next section, conditioning theory will be used to describe how deviant behaviors or fantasies might develop.

\section{Conditioning Theory of Deviant Sexual Preference and Behavior}

The Conditioning Theory of Deviant Sexual Preference and Behavior suggests that, similar to the ways that people learn acceptable behavior, they can also learn deviant behavior (Abel \& Blanchard, 1974; Laws \& Marshall, 1990; Marshall \& Eccles, 1993; Marshall \& Marshall, 2000; \& McGuire et al., 1965). Conditioning theory suggests that the repeated association of sexual fantasies and sexual pleasure (i.e., masturbation and orgasm), increases the desire to act out those fantasies in the real world (Marshall \& Eccles, 1993; Marshall \& Marshall, 2000). According to McGuire and his colleagues (1965), the development of deviant sexual preference and behavior takes place through two-steps: 1) an acquisition process and 2) a maintenance process (Abel \& Blanchard, 1974; Laws \& Marshall, 1990). These processes are outlined in the following sections. 
Acquisition processes. During the acquisition process, new behaviors develop based on the principles of conditioning theory (Abel \& Blanchard, 1974; Laws \& Marshall, 1900; McGuire et al., 1965) or social learning theory (Bandura, 1973; Laws \& Marshall, 1990). While these two theories of behavior development are not mutually exclusive, each follows a specified set of principles that can support each other (Laws \& Marshall, 1990).

Basic conditioning processes. Two conditioning processes help to explain the development of behavior, operant and classical conditioning. In general, operant conditioning involves using reward following a behavior to increase the likelihood of its future occurrence (i.e., "positive reinforcement”) or strengthening a behavior by removing a negative stimuli or outcome (i.e., "negative reinforcement"). If a sexual act is followed by sexual arousal, ejaculation, or a positive response from a partner (i.e., a reward), the frequency of that behavior is expected to increase over time (Laws \& Marshall, 1990). For example, if a young teen, who babysits his 8-year-old sister every day, gets the idea to recreate an internet porn scene with her manually masturbating him over a number of days, this may lead to him getting erections in the presence of his sister (or other similar aged girls). This may be explained by the reward of ejaculation (a powerful reward) following being in the presence of young girls.

Following the principals of operant conditioning, consistent punishment following sexual arousal to a stimulus will likely prevent an association from forming between sexual arousal and other stimuli in the environment (Laws \& Marshall, 1990). For example, if children are consistently punished for inappropriate sexual behaviors, 
masturbation in a public space, or displaying their genitals, the behavior will eventually be eliminated.

Classical, or Pavlovian, conditioning occurs when a neutral stimulus (e.g., a bell) is paired enough times with an unconditioned stimulus (e.g., food) so that the bell alone can produce salivation (i.e., conditioned response; Laws \& Marshall, 1990; Marshall \& Eccles, 1993). Initially, human sexual arousal is an unconditioned response (i.e., imagining a pretty girl naked may lead to the boy having an erection; Laws \& Marshall, 1990). Arousal can occur spontaneously or through direct stimulation of an erogenous zone (Laws \& Marshall, 1990). Over time, various neutral stimuli (e.g., an iPad) can become a conditioned stimulus for arousal (i.e., an erection) following many pairings with an unconditioned stimulus (e.g., pornographic images on the iPad; Laws \& Marshall, 1990). This process can result in the development of inappropriate or deviant behaviors. For example, a young adolescent male has recently begun experiencing sexual arousal more frequently (i.e., Unconditioned Response). One evening, the adolescent's older brother shows him a violent pornographic video where a woman is being physically held against her will (i.e., Neutral Stimuli). While watching the video, the adolescent experiences sexual arousal. Enjoying this feeling, the adolescent searches violent pornography online while doing his homework over the next week and masturbates to orgasm. A few days later, while watching television with his father, the adolescent experiences sexual arousal (i.e., Conditioned Responses) when a scene shows a woman struggling to break free of the ropes holding her to a chair (i.e., Conditioned Response) while her home is being burglarized. 
If sexual arousal is not re-paired with the unconditioned stimulus or if reinforcement does not continue, this association will weaken and disappear over time (Laws \& Marshall, 1990). This process of weakening is referred to as "extinction” (Laws \& Marshall, 1900).

Social learning theory. While conditioning processes represent one pathway in the acquisition process, behavior can also be learned through observation. Sexual behavior is a form of social behavior and can be learned from other people, like any social behavior (Laws \& Marshall, 1990). According to social learning theory, there are three major pathways by which an individual can learn a new behavior (Bandura, 1973; Laws \& Marshall, 1990). First, a new behavior can be learned through participant modeling (Bandura, 1973). During participant modeling, the learner engages in a behavior and then attempts to reenact the behavior as they experienced it (Bandura, 1973; Laws \& Marshall, 1990). In the context of sexual offending, this may be observed in the sexually abused abuser; whereby the abuser copies behaviors experienced during his own victimization (Laws \& Marshall, 1990). While participant modeling may explain the etiology of some SOs, research indicates that further explanation of the development of sexual behavior is necessary. In their meta-analysis, Seto \& Lalumiere (2010) concluded that juvenile SOs are significantly more likely to have experienced sexual abuse than juvenile NSOs. However, the meta-analysis also revealed that, on average, only approximately $46 \%$ of juvenile SOs reported sexual abuse victimization (Seto \& Lalumiere, 2010). This finding indicates that participant modeling cannot, by itself, explain the development of sexual behavior for all juvenile SOs. 
Second, behaviors can be learned through vicarious learning where the learner is not a participant in the behavior, but observes or hears about the behavior (Bandura, 1973). The learner then attempts to replicate the behavior that they have seen or heard (Bandura, 1973; Laws \& Marshall, 1990). Vicarious learning can occur in person or through visual media (e.g., video, photographs; Laws \& Marshall, 1990). Research has shown that youth verbally share a significant amount of their own sexual experiences with their peers, indicating that learning can happen through the exchange of experiential information (Epstein \& Ward, 2008). Additionally, juvenile SOs, on average, report significantly more exposure to others sexual behaviors (e.g., parents) or pornography than NSOs (Seto \& Lalumiere, 2010). Again, however, vicarious learning may only explain the etiology of a portion of juvenile sexual offending.

Finally, behaviors can be learned through symbolic modeling (Bandura, 1973). The process of symbolic modeling involves developing and expanding upon a behavior in thought (Bandura, 1973; Laws \& Marshall, 1990). Symbolic modeling is often paired with masturbation and plays a crucial role in the development and maintenance of deviant sexual fantasies (Abel \& Blanchard, 1974; Laws \& Marshall, 1990). Exposure to deviant stimuli can inform fantasies that may be expanded upon or modified over time (Abel \& Blanchard, 1974; Laws \& Marshall, 1990). Once a behavior has been acquired, either through conditioning or social learning, the behavior must be maintained to remain relevant in an individual's sexual behavior repertoire.

Maintenance processes. Once deviant tendencies have been acquired, a maintenance process occurs by which these tendencies are sustained as a part of the 
individual's behavior pattern (Laws \& Marshall, 1990). During the maintenance process, behaviors are practiced and paired with sexual arousal over time. Behaviors that have reached the maintenance process are highly resistant to change, as they have already been incorporated into the individual's socio-sexual repertoire (Laws \& Marshall, 1990). Each time sexual gratification is paired with the target stimuli, the behavior becomes more likely to be repeated. Over time, vicarious learning becomes less influential on behaviors and symbolic modeling becomes the primary mechanism by which deviant tendencies are maintained (Laws \& Marshall, 1990). Using symbolic learning, an individual may expand upon what they find to be arousing by elaborating on current scenarios or shifting arousal to variations of the effective stimuli during sexual fantasies.

An important principal of the maintenance process is the fact that intermittent reinforcement is more effective in maintaining behaviors than continuous reinforcement (Laws \& Marshall, 1990). It is much more difficult to eliminate behaviors that have been intermittently reinforced, particularly when there have been longer and more variable periods of time between reinforcement episodes (Laws \& Marshall, 1990). If a behavior is reinforced intermittently, instead of continuously, the learner continues to repeat the behavior hoping for the reinforcement. If the reinforcement is something that the learner desires, sexual gratification in this case, they will continue a behavior because of the possibility of receiving reinforcement. If they do not receive reinforcement, they will try again until they do.

Deviant sexual acts are problematic because society has deemed them inappropriate (Laws \& Marshall, 1990). This disapproval of such acts allows for the 
assumption that successful satisfaction of these desires occurs less frequently than socially acceptable sexual behaviors (Laws \& Marshall, 1990). If deviant behaviors are reinforced intermittently, as noted above, they can be expected to be persistent and more resistant to extinction processes.

\section{Summary}

In combination, the Social Deficits Model (Marshall, Hudson, \& Hodkinson, 1993) and the Conditioning Theory of Deviant Sexual Preference and Behavior (Laws \& Marshall, 1990) provide a foundation for understanding the development and maintenance of deviant sexual thoughts and behaviors. These theories offer a strong foundation for our understanding of the role of sexual fantasies and behaviors in juvenile sex offending. First, the Social Deficits Model of sexual offending behavior suggests that sexual offenders are unable to achieve intimacy or develop relationships with appropriately aged partners (Daleiden et al., 1998; Marshall et al., 1993). This inability to connect causes the offender to seek out fulfillment of intimacy through the physical act of sex and to seek sexual intimacy with inappropriate partners. Second, Conditioning Theory of Deviant Sexual Preference and Behavior state that sexual behavior is learned through temporal pairing (Abel \& Blanchard, 1973; Laws \& Marshall, 1990; Marshall \& Eccles, 1993; McGuire et al., 1965), reinforcement (Laws \& Marshall, 1990), or observation (Bandura, 1973; Laws \& Marshall, 1990). The behavior is then maintained over time by the continued pairing of the stimulus of interest and positive feedback (e.g., sexual arousal, ejaculation, positive response from a sexual partner). Together these theories suggest that juvenile SOs seek out inappropriate partners and engage in more 
deviant sexual behaviors and fantasies when compared to juvenile NSOs. In the next chapter, the role of sexual behavior in sexual offending will be discussed in more detail, followed by an in-depth discussion of the role of sexual fantasy in sexual offending. 
Chapter IV: Sexual Behavior of Juvenile Sexual Offenders

The Social Deficits Model (Marshall et al., 1993) of sexual offending and the Conditioning Theory of Deviant Sexual Preference and Behavior (Laws \& Marshall, 1990) both point to deviant sexual behavior as an important component in the development of sexual offending behavior. In a meta-analytic review, Seto and Lalumiere (2010) investigated differences between juvenile sexual offenders (SOs) and non-sexual offenders (NSOs) on a variety of significant theoretical and applied constructs. Results revealed that SOs reported greater exposure to others' sexual behavior and pornography, as well as, an earlier age of first intercourse than NSOs. Of relevance to the present study, this earlier exposure to others' sexual behavior and pornography reflects a greater number of experiences and exposures to draw from for the purposes of achieving sexual arousal (Laws \& Marshall, 1990). If these sexual experiences and exposures are negative in nature they may lead to a stronger connection between "deviant" arousal or violent fantasies and an interest in engaging in inappropriate or abusive sexual behavior (Laws \& Marshall, 1990). This chapter will describe research studies that have investigated differences between juvenile SOs' and NSOs' sexual behavior. This chapter will also discuss advances in technology that may have a critical influence on juvenile sexual behaviors that have yet to be systematically accounted for in empirical research.

\section{Male Juvenile Offenders and Sexual Behavior}

Currently, there is a paucity of research comparing the sexual behavior of juvenile SOs and NSOs. Only two peer-reviewed studies have directly compared the sexual behavior of these two groups (i.e., Daleidan et al., 1998; Fagan \& Wexler, 1988). Both 
studies are outdated, with the most recent study being completed in 1998 (i.e., Daleidan et al., 1998). While these studies provide important information, there have been several advances in technology (which will be discussed later in this chapter) that may influence these findings. First, however, the available research will be reviewed.

In the first of two peer-reviewed comparisons of juvenile SOs and NSOs, Fagan \& Wexler (1988) conducted 90-minute face-to-face interviews with and examined the court histories of 242 male violent juvenile offenders, 34 of which were identified as SOs. Interviews focused on bonds with family, work, peers, and the community, attitudes toward the law, attitudes toward violence, and prior victimization. Findings indicated that SOs were less likely to report having a girlfriend in the past 6 months, were more likely to indicate the belief that sexual relations were not an important part of an emotional relationship, were more than twice as likely to report high rates of forcing a partner to have sex, and were more likely to report "no involvement" in sexual activity than NSOs. This study indicates that juvenile SOs are less likely to report "traditional" and developmentally appropriate sexual beliefs and behaviors than juvenile NSOs.

In the second of the two peer-reviewed studies, Daleidan and his colleagues (1998) used the Sexual History Form (SHF; Kaufman, 1994) to investigate differences in sexual behavior in a sample of 302 incarcerated male, juvenile SOs and 124 incarcerated male, juvenile NSOs. Results indicated that SOs reported fewer "Typical/Consenting" behaviors (e.g., behaviors that are nondeviant - kissing, petting, and vaginal intercourse, with a consenting partner), more "Typical/Nonconsenting" behaviors (e.g., behaviors that are nondeviant - kissing, petting, and vaginal intercourse, but with a nonconsenting 
other), more "Solitary Sexual Acts" (e.g., masturbating alone, having sexual activity with animals, and looking at pornographic pictures), and more "Paraphilic" (e.g., masturbating in a public place, becoming sexually excited by watching fire) behaviors than NSOs. These findings provide support for Fagan \& Wexler's (1988) conclusions, indicating that juvenile SOs have fewer nondeviant sexual experiences and more deviant sexual experiences than NSOs.

The lack of research investigating differences in the sexual behavior of juvenile offenders indicates a gap in empirical knowledge on this topic. The outdated nature of the existing research remains problematic due to significant advances in technology over the past two decades. Clearly, these advances impact the way that juveniles access sexual images and experience sexual development, highlighting the need for additional research in this area.

\section{Advances in Technology and Sexting}

There have been several crucial advancements in technology that likely influence the development of adolescent sexuality. Since the publication of the two relevant articles in this area (i.e., Daleiden et al., 1998; Fagan and Wexler, 1988), the influence of technology and easier access to pornography have become of greater interest to researchers in the field. A survey conducted in 2018 revealed that up to $88 \%$ of teens have access to a desktop or laptop computer (Anderson \& Jiang, 2018). In addition, 45\% of teens report that they use the internet "almost constantly" and another $44 \%$ report they go online several times a day (i.e., 9-10 times a day), figures that have almost doubled since 2014-2015 (Anderson \& Jiang, 2018). The percentage of homes with high-speed 
broadband internet access in the home has increased from about $19 \%$ at the end of 2003 to $75 \%$ at the beginning of 2019 (Anderson, 2019). A greater number of homes with access to computers and high-speed internet creates increased, instant access to online pornography and social networking over the last two decades.

There has been a significant shift in how pornography is accessed, from home video and television, to the internet and smartphones (Price et al., 2016). The internet and smartphones allow pornography to be distributed to a broader audience, facilitated by three critical factors: anonymity, affordability (competitors keep prices low with plenty of "free" options), and accessibility (i.e., 24/7 availability; Cooper, 1998). Using data from the General Social Survey (GSS), Price and his colleagues (2016) found that there is a significant gap in consumption of pornography between those born before the advent of the internet and those born after, with those who were born after the internet reporting higher use of pornography (about 10\%), than those who were a part of the 70s cohort (Price et al., 2016). In addition, data showed an increase in consumption of pornography over time, with $44.9 \%$ of males reported having "seen an X-rated movie in the past year" in the 1970 s and about $61 \%$ in 2000s (Price et al., 2016). This finding supported findings by Wright (2013) who, using the same GSS data set, concluded that although increases were incremental, average pornography consumption has continued to rise with $25 \%$ of participants reporting that they viewed pornography in the 1970 s and $34 \%$ in the 2000 s. Differences in methodology were discussed as being the reason for disparities among the two studies. For example, Price et al. (2016) were specifically testing for age, cohort, and period effects, which Wright (2013) did not do. In addition to the internet creating easier 
access to pornography, a vast majority of teens own a cell phone, making this access mobile and more private.

Evidence suggests that approximately $95 \%$ of youth between the ages of 12 and 17 own a cell phone, up from 73\% in 2014-2015 (Schaeffer, 2019). Cell phones provide instant access to pornography and the internet; as well as, communication with peers and strangers through text messaging, email, and social media. The use of cell phones has become an integral component in modern day-to-day living. So much so, that research has suggested that the current generation experiences a significant part of their social and emotional development and sexual exploration through text messaging and social media (Cooper et al., 2016; O’Keefe \& Clarke-Pearson, 2011).

A phenomenon known as "sexting" has developed through advances in technology. Sexting has been defined as: "youth writing sexually explicit messages, taking sexually explicit photos of themselves or others in their peer group, and transmitting those photos and/or messages to their peers." (National Conference of State Legislatures, 2009). While individuals of all ages can sext, it has become a major concern for our youth. The prevalence of sexting among adolescents is not well understood, as numbers differ across studies depending on factors such as definitions, country, and age ranges of participants (Cooper et al., 2016). It is estimated that $12 \%$ of American adolescents have sent sexts and that there is an increase in sexting with age (Buren \& Lunde, 2018).

The practice of sexting can have far reaching, negative legal ramifications for youth. Section 2256 of Title 18, United States Code, defines child pornography as "any 
visual depiction of sexually explicit conduct involving a minor" (United States Department of Justice, 2015). Additionally, the United States Department of Justice (DOJ) defines a minor as someone under the age of 18 (United States Department of Justice, 2015). Therefore, while adolescents are engaging in sexting, often with same age peers and sometimes in the context of romantic relationships, the photographs being exchanged have been determined to constitute child pornography.

Many adolescents engage in sexting with same-age peers without fully understanding the legal ramification of their actions. While sexting encompasses a range of motives (e.g., consensual sharing, coerced sharing; Judge, 2012), even consensual sexting can legally result in the adolescent being in possession of illegal "sexually explicit" material and can lead them to face charges. It is difficult to determine how many cases involving juvenile sexting are prosecuted nationally. In an exploratory study, Walsh et al. (2013) asked 236 prosecutors in state courts, who have handled sexting cases involving juveniles, about their practices. Many cases were provided alternatives to being charged (e.g., counseling, community service, loss of technology; Walsh et al. 2013). However, $21 \%$ said that most or all of their cases ended with charges filed and of those prosecutors, 62\% charged juveniles with felony counts (Walsh et al., 2013).

In a recent review of sexting behaviors, adolescents reported engaging in sexting to flirt or gain attention from someone they were interested in, as a way to maintain intimacy during periods of physical separation from their partner, and as a means of expressing mutual affection and arousal in anticipation of physical intimacy (Cooper et al., 2016). While each of these behaviors represents consensual actions, Cooper and her 
colleagues (2016) found that adolescents often engage in sexting due to pressure from a romantic partner or their perceptions of sexting as normative among peers. Females also report pressure to produce sexual images for romantic partners more often than males and males tended to report pressure from peers more often than females. Finally, Cooper and her colleagues (2016) found that evidence is emerging that individuals who have engaged in sexting are more likely to be sexually active, to have engaged in sexual behavior at an earlier age, and to have taken part in recent "high-risk" sexual behaviors (e.g., multiple partners, unprotected sex). Often, pressure from peers and romantic partners can lead to non-consensual image sharing (i.e., sexting; Cooper et al., 2016).

One significant risk of engaging in sexting is the nonconsensual sharing of sexual images outside of a consensual relationship (Cooper et al., 2016). Non-consensual image sharing, also referred to as "revenge porn" can have immense psychological consequences for the victim whose image was shared (Cooper et al., 2016). Often times, revenge porn occurs when someone who has received consensual images distributes that image without the senders' knowledge, indicating a disconnect between the sender's intentions and the distribution of these images (Cooper et al., 2016). This nonconsensual sharing can lead to bullying through threats and blackmail (Cooper et al., 2016). Research on non-consensual image sharing is lacking in general, but even more so within the context of adolescent behavior (Cooper et al., 2016).

The phenomenon of sexting is a new construct of interest to the field of child sexual abuse. Due to this area of research being in its infancy, peer-reviewed research on sexting is severely lacking (Judge, 2012). What is also unclear is whether sexting has 
become a normalized phenomenon, or if it is a behavior that SOs might be engaging in at a higher rate than NSOs. Building upon the work of Daleiden and his colleagues (1998) and Fagan \& Wexler (1988), the current study seeks to incorporate the investigation of sexting in adolescents into a broader examination of juvenile offenders' sexual behavior.

\section{Summary}

According to the Conditioning Theory of Deviant Sexual Preference and Behavior (Laws \& Marshall, 1990), having earlier exposure to sex and pornography would increase the potential for an adolescent to become a sexual offender. While sexual behavior is a critical factor being addressed in juvenile SO treatment, a lack of recent research examining how sexual behavior may differ for SOs, as opposed to NSOs, inhibits the field's ability to understand factors critical to sexual etiology in juvenile offenders. Further, research focused on the influence of advances in technology on sexual offending is also lacking. This study will begin to fill these critical gaps in the research literature. The next chapter will discuss the role of sexual fantasy in sexual offending behaviors. 
Chapter V: Sexual Fantasies of Juvenile Sexual Offenders

Sexual fantasies have been identified as an important factor in the development and maintenance of sexual deviation (Abel \& Blanchard, 1974; Bartels \& Gannon, 2011; Gee et al., 2003; Hunter \& Becker, 1994; Kenny et al., 2001; Langevin et al., 1998; Worling \& Langstrom, 2006). Sexual fantasies represent "almost any mental imagery that is sexually arousing or erotic to the individual” (Leitenberg \& Henning, 1995, p. 470). Similarly, Rokach (1990) defines sexual fantasy as "any daydreaming that includes erotica and that is sexually stimulating" (p.427). For this study, sexual fantasies are defined as "thoughts, feeling, or images that include sexual activities and feelings (Kaufman, 1998).” Leitenberg and Henning (1995) identify the critical component of an intentional sexual fantasy as the individual's ability to control exactly what takes place in the imagination. Fantasies can be derived from personal experience, or something that is seen, heard, or read (Gee et al., 2004; Marshall \& Marshall, 2000). Not only can fantasies influence future behavior, but fantasies can also be affected by previous experiences (Leitenberg \& Henning, 1995).

Sexual fantasizing was first normalized by the work of Kinsey, beginning in the 1940s, when he and his colleagues reported that both men and women frequently have sexual fantasies (Rokach, 1990). Since then, research has been interested in the importance of sexual fantasy in the etiology of sexual offending. Researchers have explored the development (Hunter \& Becker, 1994), function (Gee et al., 2003), content (DiGiorgio-Miller, 2007; Gee et al., 2004), and group differences in sexual fantasies for sexual offenders (SOs; Daleiden, et al., 1998; Rokach, 1990). In this chapter, sexual 
fantasies in the non-offending population will be discussed, followed by an exploration of the role of sexual fantasy in adult, male sexual offending behavior. Finally, a review of the current literature on sexual fantasies and juvenile SOs will be provided.

\section{Sexual Fantasy in Non-Offending Populations}

In a seminal review of sexual fantasy, Leitenberg and Henning (1995) reported that across 13 studies reviewed, on average, $85.9 \%$ of men and $68.8 \%$ of adult women report having had sexual fantasies during masturbation at some time in their life. Across 12 studies, on average, $76 \%$ of men and $70.1 \%$ of women reported sexual fantasies during intercourse at some time in their life. Finally, across 6 studies, on average, $93 \%$ of men and $84.8 \%$ of women reported ever having sexual daydreams (i.e., sexual fantasies while not engaged in sexual activity). These data make it apparent that sexual fantasizing is a common occurrence across genders in the non-offending population. In fact, sexual fantasizing has been identified as a critical component of healthy sexuality (Maniglio, 2011).

More recently, an exploration of the content of sexual fantasies that occur in the non-offending populations was undertaken by Joyal and colleagues (2015). Researchers disseminated an online survey that was completed by 1,516 adults. Each participant was asked to rank 55 predetermined sexual fantasies on an extended version of the Wilson Sex Fantasy Questionnaire (Wilson, 1988). Each fantasy was categorized as "statistically rare" (2.3\% [2 standard deviations below the mean] endorsement or less), "unusual" (15.9\% [1 standard deviation below the mean] endorsement or less), "common" (more than $50 \%$ [the mean] endorsement), or "typical" (more than $84.1 \%$ [one standard above 
the mean] endorsement). It was not clear if participants who reported no fantasies were included in analyses.

Results indicated that across gender, only two sexual fantasies were found to be statistically rare and nine sexual fantasies were found to be unusual (Joyal et al., 2015). For both genders, sex with a child under the age of 12 and sex with an animal were found to be statistically rare (Joyal et al., 2015). There were some gender differences found when identifying unusual behaviors. For both genders, urinating on a partner and being urinated on were found to be unusual (Joyal, et al., 2015). For women, wearing clothes of the opposite gender, forcing someone to have sex, abusing a person who is drunk, asleep, or unconscious, having sex with a prostitute, and having sex with a woman who has very small breasts were found to be unusual (Joyal et al., 2015). For men, having sex with two other men and having sex with more than three other men were found to be unusual (Joyal et al., 2015). These results suggest that very few fantasies should be determined to be "rare" or "unusual" in non-offending populations.

\section{Sexual Fantasy in Sexual Offending}

It has long been assumed that deviant sexual fantasies play a critical role in sexual offending behavior (Aylwin et al., 2005; Bartels \& Gannon, 2011; Hunter \& Becker, 1994; Kenny et al., 2001). According to the Social Deficits Model (Marshall et al., 1993) of sexual offending and the Conditioning Theory of Deviant Sexual Preference and Behavior (Laws \& Marshall, 1990), deviant fantasies that are reinforced, through masturbation or positive response, have a higher likelihood of manifesting in overt 
behavior (Laws \& Marshall, 1990; McGuire et al., 1965). In this section, the role and content of sexual fantasies in sexual offending behavior will be described.

The function of sexual fantasies. In order to better understand the function of sexual fantasy for SOs, Gee and colleagues (2003) developed the Sexual Fantasy Function Model (SFFM) using a sample of 24 adult, male SOs who were incarcerated at a correctional facility at the time of interviews. Using a grounded theory methodology, these researchers found that sexual fantasy serves four primary functions in the process of sexual offending (Gee et al., 2003). The first of these functions, according to the SFFM, is affect regulation. Sexual fantasy impacts affect regulation, focused specifically on the offender's mood, by alleviating feelings of depression, elevating an offender out of boredom by increasing internal stimuli, and enhancing positive feelings often leading to sensation seeking behavior (Gee et al., 2003). The second function of sexual fantasy is the regulation of sexual arousal. Typically acting as a precursor to masturbation, sexual fantasy is used to induce a state of sexual arousal (Gee et al., 2003). Sexual fantasy can also be used to enhance current sexual arousal and to reach orgasm (Gee et al., 2003). The third function of sexual fantasy is that of coping (Gee et al., 2003; Maniglio, 2011). This is focused on being able to control or manipulate scenarios to justify behavior or to detach from their present situation (e.g., imagining an ideal partner while having sex with their wife; Gee et al., 2003). Finally, sexual fantasy provides an opportunity to plan for future acts by creating offense scripts or to relive past sexual activity, including the emotional and physiological experience (Gee et al., 2003). In addition to the function of 
sexual fantasy, Gee and colleagues (2004) were interested in the content structure of sexual fantasies held by SOs. Their findings are described below.

The content of sexual fantasies. Using a sample of 24 adult, male SOs, who were either in a correctional facility or one of two community-based SO treatment programs, Gee and colleagues (2004) developed The Sexual Fantasy Content Model (SFCM). The SFCM was developed to foster an accurate description of SOs' sexual fantasies throughout the offense process. The SFCM contains three higher-order categories: 1) general sexual fantasy; 2) non-specific offense-focused fantasy; and 3) specific offense-focused fantasy. Within each of the three higher-order categories, the SFCM has five, second order categories: 1) demographic; 2) behavioral; 3) relational; 4) situational; and 5) self-perceptual. General sexual fantasy refers to sexual behavior that is not related to the commission of a sexual offense and is comprised of behaviors that are both sexual and emotional in nature (e.g., "I had fantasies of oral sex with friends growing up."). In this sample of offenders, $92 \%$ of participants who reported the use of sexual fantasy, used general sexual fantasy. Non-specific offense-focused fantasy refers to fantasies that are general in nature and if acted upon, would legally constitute a sexual offense (e.g., "I had fantasies about young girls, but not anyone in particular."). In this sample, $75 \%$ of participants reported using non-specific offense-focused fantasies. Finally, specific offense-focused fantasy refers to fantasies about specific offense characteristics, which if acted upon, would constitute a sexual offense (e.g., "I had thoughts about this particular boy.”). In this sample, all participants who reported the use of sexual fantasy, indicated that they used specific offense-focused sexual fantasies. 
The five second-order categories relate to the specific content of the fantasy and together form the theme of the sexual fantasy (Gee et al., 2004). The demographic subcategory refers to the gender, age, and biosocial relationship of the individuals in the fantasy. Often, these characteristics mirror real life; however, these characteristics can also be altered abstractions of real life (e.g., "I had thoughts about my step-daughter, but in my head, she was a lot older, she was an adult."). The second subcategory, behavioral characteristics describe the sexual activity that takes place during the fantasy. The relational subcategory refers to how the individuals in the fantasy interacted with each other. The situational subcategory refers to when and where the fantasy was taking place. Finally, the fifth subcategory, self-perceptual, refers to how the individual having the fantasy perceived himself during the fantasy (e.g., "I am in control, she is doing everything I am telling her to do.”).

While the specific role and content of sexual fantasies are unique to each individual, the research conducted by Gee and her colleagues (2003; 2004) provides a structure for the conceptualization of sexual fantasy in sexual offending behaviors. One of the biggest gaps in the field is the lack of research specific to juvenile sexual offending (Alywin et al., 2005; Hunter \& Becker, 1994; Langevin et al., 1998). It is often the case that research conducted on adult SO samples is applied to juvenile SOs (Hunter \& Becker, 1994). In addition, findings that result from exploring the importance of sexual fantasy to sexual offending behaviors for adults have been mixed (Maniglio, 2011). Simply speaking, this finding indicates that having a sexual fantasy does not necessarily 
mean it will be acted on (Maniglio, 2011). The next section will discuss the available research on the sexual fantasies of juvenile SOs.

\section{Male Juvenile Offenders and Sexual Fantasy}

Much of the research on the influence of sexual fantasy on sexual offending has been conducted with adult male samples (e.g., Gee et al., 2004; Gee et al., 2003). In fact, very little research has been conducted with juveniles in this area (Alywin et al., 2005; Hunter \& Becker, 1994; Langevin et al., 1998). A search of the research literature returned a limited number of peer-reviewed research articles investigating the sexual fantasies of juvenile SOs and only one comparing juvenile SOs and NSOs.

In a study of 33 inpatient and 33 outpatient juvenile SOs, DiGiorgio-Miller (2007) used the Sexual Fantasy Questionnaire (SFQ; Kaufman, 1994) to explore the relationship between treatment settings, sexual fantasies, and mood states. DiGiorgio-Miller (2007) found that SOs involved in inpatient treatment reported a greater number of deviant and non-deviant sexual fantasies than SOs in outpatient treatment. The author did not, however, discuss which subscales of the SFQ were used in this study. In the combined sample of inpatient and outpatient SOs, it was found that the number of deviant fantasies reported were positively correlated with the number of offenders' victims, their number of offenses, their non-deviant fantasies, and their feelings of hostility. DiGiorgio-Miller (2007) also found a positive correlation between the number of non-deviant sexual fantasies and age.

In a review of the factors related to the development or maintenance of deviant sexual arousal for juvenile SOs, Hunter and Becker (1994) identified three important 
experiences. Prior maltreatment experiences, exposure to sexually explicit materials and substance abuse, and exposure to aggressive role models all appear to be prominent in the histories of juvenile SOs (Hunter \& Becker, 1994). Conditioning Theory of Deviant Sexual Preference and Behavior would suggest that these experiences create a plethora of deviant behaviors and activities to draw from in informing their sexual fantasies (Laws \& Marshall, 1990; McGuire et al., 1965). While this information may play a critical role in informing the etiology of juvenile SOs, it is important to compare juvenile SOs to NSO control groups to determine which factors differentiate the two groups. Unfortunately, a search of the literature exploring the differences between juvenile SOs and NSOs revealed a lack of research with this focus.

Only one peer-reviewed research study has compared juvenile SOs and NSOs on sexual fantasies. Daleidan and his colleagues (1998) used the SFQ (Kaufman, 1998) to test differences in a sample of 302 incarcerated male SOs and 124 male NSOs. Findings indicated that SOs reported a greater number of "Deviant" and "Nontraditional" sexual fantasies than NSOs. Sexual fantasies that comprised the "Global Deviance" subscale included fantasies such as whipping, beating, or torturing other and fantasizing about paraphilias. Sexual fantasies that comprised the "Nontraditional Fantasies" subscale included fantasies such as exposing sexual parts and looking at pornographic pictures of oneself.

\section{Summary}

Deviant sexual fantasies have been targeted as important for prevention, as well as treatment efforts, for some time. However, what would traditionally be termed as 
deviant fantasies are found to occur frequently in non-offending samples (Bartels \& Gannon, 2011; Joyal et al., 2015; Leitenberg \& Henning, 1995). This suggests that many such behaviors may not be as deviant as they have been assumed to be in the past. Conditioning Theory of Deviant Sexual Preference and Behavior suggest that sexual fantasies are an important part of the development and maintenance of deviant behavior (Laws \& Marshall, 1990; McGuire et al., 1965). A paucity of research and insight into the role of sexual fantasies for juvenile SOs indicates that it is an important area for pursuing additional research. The next chapter will discuss the role of sexual fantasy and sexual behavior in prevention and treatment. 
Chapter VI: The Role of Sexual Fantasy and Sexual Behavior in Prevention and Treatment

For decades, prevention and treatment efforts have focused on the role of sexual fantasy and sexual behavior in sexual offending (Abel \& Blanchard, 1974). Following the public health approach, these constructs can be used to inform both secondary and tertiary treatment efforts. While theoretical literature supports the targeting of these constructs during treatment, the empirical evidence to support these directions has been slow to emerge. This chapter will discuss the role of sexual fantasy and sexual behavior in prevention and treatment efforts directed toward juvenile sexual offenders (SOs). Each construct will be discussed, more specifically, in the context of treatment and its potential to impact juvenile SO recidivism rates. Finally, a critique of these constructs as prevention and treatment targets are presented.

\section{The Significance of Sexual Fantasy and Sexual Behavior to Prevention Efforts}

The public health approach to prevention focuses on the overall health and wellness of a population, as opposed to any one individual (Centers for Disease Control and Prevention, 2004). The public health approach outlines three levels of prevention: 1) Primary; 2) Secondary; and 3) Tertiary. Sexual fantasy and sexual behavior represent etiological factors that can inform prevention efforts at the secondary and tertiary levels. The goal of primary prevention is to reach youth and the wider community to reduce risk factors (e.g., limiting access to internet and mobile devices), increase protective factors (e.g., supervision and education on boundaries), and provide them with the necessary 
tools and information (e.g., identifying grooming behavior) to avoid becoming a victim of CSA (Centers for Disease Control and Prevention, 2004; 2010).

Secondary prevention targets individuals who are already at risk and intervenes before any abuse has occurred (Centers for Disease Control and Prevention, 2004). Of relevance to this study, research on sexual fantasy and sexual behavior may uncover patterns of problematic sexual behaviors and sexual fantasies that are likely to increase the risk of sexual offending. These patterns, along with other known risk factors, can be identified in youth who may be at-risk for offending.

Tertiary prevention efforts take place after abuse has occurred and focus on preventing future incidences of abuse (Centers for Disease Control and Prevention, 2004). In the context of sexual offending, tertiary prevention typically involves the treatment of offenders to prevent reoffending. For example, offenders can work with their therapist to identify sexual behaviors and sexual fantasies that may have contributed to their offending behavior. Once these behaviors and fantasies have been identified, the therapist and offender can work together to develop approaches to prompt early recognition of these risky patterns as well as strategies to help avoid engaging in future offending behavior. In the next section, the role of sexual fantasy and sexual behavior in treatment efforts and their efficacy as treatment targets will be discussed.

\section{The Significance of Sexual Fantasy and Sexual Behavior to Treatment Efforts}

For decades, sexual fantasy and sexual behavior have been the focus of evaluation and treatment efforts (Abel \& Blanchard, 1974). Although evidence remains mixed as to the efficacy of these constructs as treatment targets, professionals have relied on 
theoretical literature to guide their practice. To understand the decision to focus on these phenomena in treatment, the available treatment frameworks that influence how to treat SOs will be reviewed. Sexual fantasy and sexual behavior will also be discussed in relation to juvenile SO assessment and treatment efforts.

Generalists vs. specialist offenders. Recently, a distinction was made differentiating two unique approaches to working with juvenile SOs. Some experts in the field believe that it is most effective to treat juvenile SOs as "generalist" offenders and others believe that it is most effective to treat juvenile SOs as "specialist" offenders (Harris et al., 2009; Pullman \& Seto, 2012). The generalist perspective suggests that: “crimes committed by [juvenile SOs] are a manifestation of general delinquent tendencies, in which sexual offenses constitute only a part of their antisocial and criminal behavior" (Pullman \& Seto, 2012 p 204). The specialist perspective, however, suggests that: "[juvenile SOs] differ from other adolescent offenders, and different factors explain sexual offending compared to nonsexual offending" (Pullman \& Seto, 2012 p 204). In other words, juvenile SOs represent a qualitatively different group of perpetrators than those identified as delinquents.

Traditionally, juvenile SOs have been conceptualized as a "special kind" of juvenile offender that has distinct risk and etiological factors (Pullman \& Seto, 2012). This conceptualization is most aligned with the specialist perspective on sexual offending. Pullman and Seto (2012) suggest that for treatment to be most effective, it should be matched to offender type and address the etiological factors specific to that type of offending behavior. For example, Pullman and Seto (2012) identify deviant 
sexual interests as an etiological factor specific to juvenile SOs that may be an appropriate treatment target. Traditionally, juvenile SOs have been treated using this specialist framework. However, Seto and Lalumiere's (2010) meta-analysis concluded that most juvenile SOs are generalist offenders who are more similar in nature to juvenile NSOs. Unfortunately, treatment effectiveness research for juvenile SOs using these two frameworks is limited.

Harris and colleagues (2009) conducted a study comparing specialist and versatile (generalist) offenders. Participants were identified as specialist offenders if at least 50\% of their crimes were classified as sexual offenses. Participants were classified as versatile (generalist) offenders if less than $50 \%$ of their crimes were classified as sexual offenses. Findings revealed that specialist offenders were more likely to show evidence of sexual preoccupation and emotional congruence with children. However, findings were limited by the participant sample, which only included adult rapists and child molesters. The limited, available research indicates mixed results for supporting the treatment of juvenile SOs as specialist offenders. The mixed nature of these results further supports the need for more research comparing juvenile SOs and NSOs to better identify similarities and differences between these groups. The present study seeks to investigate two variables that have been identified in the theoretical literature as constructs of interest to consider when identifying etiological differences between SOs and NSOs.

Sexual behavior as a treatment target. Prior sexual experiences have been identified as an important precipitator of the emergence of sexual offending behaviors (McGuire et al., 1965). The examination of sexual behaviors across the life span as part 
of the treatment process has been identified as important for two primary reasons: 1) deviant behavior may be the result of conditioning that has reinforced inappropriate behaviors (Abel \& Blanchard, 1974; MGuire et al., 1965) and 2) the deficit model of sexual offending suggests that inappropriate behaviors result from failed attempts to achieve intimacy in developmentally appropriate ways (Marshall et al., 1993). In addition to theoretical support for sexual behavior as a significant treatment target, studies have found relationships between sexual behavior and sexual recidivism.

Sexual recidivism has been indirectly linked to prior sexual experiences. Kenny and colleagues (2001) found, using a sample of juvenile SOs, that deviant sexual experience had a direct causal link to deviant sexual fantasies indicating that prior sexual experiences are informing fantasies. In turn, researchers also found that deviant fantasies had a direct causal impact on recidivism (Kenny et al., 2001). These findings suggest that future research should investigate both sexual fantasies and sexual behavior, as they both impact an offenders' likelihood to recidivate.

Sexual fantasy as a treatment target. Worling and Langstrom (2006) identified deviant sexual interests as an empirically supported risk factor for sexual reoffending (i.e., Kenny et al., 2001; Schram et al., 1992; Worling \& Curwen, 2000). Kenny and colleagues (2001) reported that adolescents who had sexually recidivated were more likely to report sexual fantasies that included the use of force and children under the age of 10. However, their investigation of sexual fantasies was limited in scope. This study only asked participants about fantasies related to the offense that they were adjudicated 
for. Additionally, the study was conducted in Australia where ideas of what is deviant might be different than in the United States.

Further, Worling \& Langstrom (2006), identified sexual preoccupation as an area of focus for future research. "Sexual preoccupation" would include sexual fantasies as well as other behaviors (e.g., sexual "daydreams"). While sexual preoccupation has been identified as a promising risk factor for adult sexual offense recidivism, it has not been examined with juvenile SO samples (Worling \& Langstrom, 2006). However, compulsive, deviant masturbatory fantasies have been noted as an important area of inquiry when working with juvenile SOs (Worling \& Langstrom, 2006).

In a comparison of 33 inpatient and 33 outpatient, juvenile SOs, DiGiorgio-Miller (2007) found that inpatient SOs reported more deviant sexual fantasies than outpatient SOs. DiGiorgio-Miller (2007) went on to suggest that juvenile SOs who are at greater risk of reoffending are traditionally recommended for inpatient treatment. The findings that inpatient juvenile SOs reported more deviant sexual fantasies than outpatient SOs would support this group's increased risk of reoffending.

In a sample of 87 male, juvenile SOs in residential treatment, Aylwin and colleagues (2005) asked participants to record their sexual fantasies during their time in therapy. The average length of stay at the residential treatment facility was 31.5 weeks with no stays longer than 12 months. In addition to recording all normal and deviant fantasies, participants were asked to indicate which fantasies were interrupted (i.e., the fantasy did not culminate in orgasm) and which fantasies were coupled with masturbation. Results indicated that reports of deviant fantasies were low at the 
beginning of treatment with very little effort to interrupt these fantasies while reports of normal fantasies were high. The report of deviant fantasies increased significantly (i.e., by $380 \%$ ) over the first five months of treatment and then steadily declined over the juvenile's remaining time in treatment. These findings support the notion that patients typically enter treatment underreporting the frequency of deviant fantasies and as they become more comfortable over time, they are more honest in their reporting of this type of information (Aylwin et al., 2005). However, the researchers were unable to determine whether the decline in the reporting of the deviant fantasies after the initial increase was due to honesty in reporting or effective treatment. While some treatment experts identify sexual fantasy and sexual behavior as important targets during treatment, findings about their effectiveness in reducing recidivism are mixed. The next section will critique the literature which assesses the impact of targeting sexual fantasy and sexual behavior during juvenile SO treatment.

\section{Treating Deviant Sexual Arousal}

In a seminal review of treatment options for juveniles who have offended sexually, Worling (2012) identified 5 primary approaches for treating deviant sexual arousal: behavioral procedures to extinguish deviant sexual arousal, behavioral procedures to increase nondeviant sexual arousal, thought suppression, mindfulnessbased cognitive therapy, and pharmacological interventions. Covert sensitization is a behavioral procedure intended to extinguish deviant arousal patterns, by asking the client to pair deviant sexual thoughts with aversive consequences (Worling, 2012). A second behavioral technique to extinguish deviant sexual fantasies is masturbatory satiation 
(Marshall, 1979, as cited in Worling, 2012). In this approach, the client is asked to masturbate to a nondeviant sexual fantasy and then, quickly after, masturbate to a deviant sexual fantasy (Worling, 2012). The intention behind this approach is for the client to associate deviant sexual fantasies with decreases arousal (Maletzky, 1991, as cited in Worling, 2012). Directed masturbation has been used as an approach to increase nondeviant sexual arousal, which involves the client masturbating only to nondeviant sexual fantasies (Maletzky, 1991; as cited in Worling, 2012). Thought stopping is intended to suppress deviant thoughts in the moment (Worling, 2012). A mindfulnessbased cognitive therapy approach asks clients to notice deviant thought and monitor how their body responds (Worling, 2012). Clients are asked to notice the urge build within them and notice it subsides (Worling, 2012). Finally, pharmacological interventions use medication to reduce deviant sexual arousal (McGrath et al., 2010, as cited in Worling, 2012).

\section{Critiques of Sexual Fantasy and Sexual Behavior as Treatment Targets}

Conclusions as to the importance of sexual fantasy and sexual behavior as treatment targets are mixed in the literature. While Seto and Lalumiere (2010) point to the significant role of sexual fantasy and sexual behavior in juvenile sexual offending in the theoretical literature, clinical practice is still relegated to making assumptions about the importance of these areas to effective treatment. Similarly, clinicians must rely on "educated guesses" as to which specific sexual fantasy and sexual behavior dimension should be selected as the focus of a youth's treatment planning. One key concern stems from evidence that the assessment and treatment of juvenile SOs has been primarily 
based on research conducted with adult SOs (Hunter \& Becker, 1994). At the same time, however, ignoring the significant differences between adult and juvenile SOs may lead to ineffective treatment approaches.

The centrality of sexual fantasy as an etiological factor in sexual offending is an assumption that is almost universally made by clinicians and is based on early research with extremely violent SOs (Daleidan et al., 1998; Howitt, 2004). This assumption may lead to an overestimate of the importance of fantasy in sexual offending (Howitt, 2004). Additionally, evidence suggests that deviant fantasies are not as common as treatment professionals assume. Using a sample of 201 adult, male SOs and controls, Langevin et al. (1998) found a base rate of deviant fantasies low enough to suggest that it is implausible for fantasy to be of etiological significance and instead only has mild utility in SO assessment and treatment. Similarly, in a sample of 221 juvenile SOs who had participated in both residential- and institutional-based treatment settings in Washington and colleagues (1991) did not find deviant arousal patterns to be predictive of risk for recidivism.

The review conducted by Worling (2012) concluded that a significant lack of empirical research exists supporting the effectiveness of treatment approaches to address deviant sexual arousal discussed above. Many of these techniques were developed for use with adult males, leaving many questions about their effectiveness with adolescents (Worling, 2012). The exception being mindfulness-based cognitive therapy techniques, which has shown greater behavioral changes when compared to suppression techniques (Rogojanski et al., 2011). Research has shown mixed results that juveniles that engage in 
sexual offending show a deviant arousal pattern, with estimate being between 25 and 30\% (Seto et al., 2000;2003). Therefore, better understanding the role of sexual behavior and sexual fantasy in sexual offending is important to informing treatment efforts.

\section{Summary}

There is little evidence supporting the efficacy of efforts to reduce deviant sexual fantasies and behaviors and the few studies that have endeavored to do so have used overall program outcomes as an indicator of success (Howitt, 2004; Hunter \& Becker, 1994). It is important to further investigate the role of sexual fantasy and sexual behavior in sexual offending to determine its effectiveness as a treatment target for juvenile SOs (Howitt, 2004). The present study seeks to identify differences in sexual fantasy and sexual behavior, between juvenile SOs and NSOs to determine whether these variables are of etiological importance for the prevention and treatment of sexual offending. 
Chapter VII: Critiques of the Current Literature and Purpose of the Present Study

There is a paucity of research focused on understanding the differences between juvenile sexual offenders (SOs) and non-sexual offenders (NSOs; Hunter \& Becker, 1994; Leitenberg \& Henning, 1995; Seto \& Lalumiere, 2010). The research literature that does exist does not allow for conclusions to be drawn with confidence regarding factors that differentiate these two groups. Limitations to the current body of literature are both methodological in nature, and due to the small number of relevant studies that have been completed in this area. The present study is intended to enhance our understanding of differences between these two groups and highlight areas that may be of relevance to guide effective prevention and intervention initiatives. Additionally, the present study will further clarify the pertinence of sexual fantasy and sexual behavior as etiological constructs of interest for juvenile SOs. This chapter provides a critique of the current body of literature, in greater detail, as well as describes the purpose of the current study. In addition, a more detailed discussion of how this study can provide findings to address significant gaps in the current literature are included.

\section{Critiques of the Existing Literature on Juvenile Offenders}

Over the past decade, there has been a call in the research literature for more studies that compare juvenile SOs and NSOs on relevant dimensions (Hunter \& Becker, 1994; Leitenberg \& Henning, 1995; Seto \& Lalumiere, 2010). It is assumed that additional work in this area will improve our understanding of juvenile sexual offending and how it may or may not differ from other types of offending. This enhanced 
understanding will result in better-informed prevention efforts and more effective treatment planning.

The current literature on the sexual fantasies and sexual behavior of juvenile SOs is limited in several important ways. First, the only existing peer-reviewed studies comparing juvenile SOs' and NSOs' sexual behavior and/or sexual fantasy, are now almost 28 and 18 years old (e.g., Fagan \& Wexler, 1988; Daleidan et al., 1998, respectively). These studies were conducted at a time prior to relevant advances in technology (e.g., webcams, cell phones, social media) and the subsequent increase in the number of youth with cell phones. These technological advances are not accounted for in the available research on this topic. Advances in technology could not only change the way that sexual behavior and sexual fantasy is conceptualized but may change the relative degree of influence these two constructs may have on sexual offending behavior. It is concerning that such a lack of research exists focusing on constructs that treatment professionals emphasize.

Second, methodological limitations impact the ability to draw conclusions with confidence and to generalize study findings to the larger population of juvenile SOs. Much of the work done with juvenile SOs is based on findings from research studies that use exclusively adult samples (e.g., Gee et al., 2004; Langevin et al., 1998). While information based on adult samples provides interesting information and a place to start when developing research questions for juvenile samples, one cannot assume that findings on adult SOs will closely approximate those of juvenile SOs. Moreover, the methodology used in each study can limit the generalizability of findings. 
Third, research on the sexual fantasies and sexual behavior of juvenile SOs is often limited to samples that have been recruited from residential treatment facilities (e.g., Aylwin et al., 2005; DiGiorgio-Miller, 2007). While this research is important, juvenile SOs who are adjudicated to a correctional facility tend to represent offenders at a greater risk for reoffending (DiGiorgio-Miller, 2007). As such, this group may represent a distinct subpopulation of juvenile SOs whose treatment is being based primarily on research done in residential treatment facilities. While some components of treatment may be the same for offenders adjudicated to correctional facilities and those who are adjudicated to residential treatment, the ways in which it is delivered may be different (i.e., emphasizing different areas of the curriculum). Due to these limitations, there is much to study related to this area. The current study has been designed to begin to address these methodological and content-based gaps.

\section{Purpose of the Present Study}

Although research on this population is lacking, sexual fantasy and sexual behavior have been used as treatment targets for decades. The purpose of the present study was to investigate the differences in the sexual fantasies and sexual behavior of juvenile SOs and NSOs to enhance the quality of this literature. A recent literature review suggests that this research project will be the first study to attempt to identify information related to sexual fantasy and sexual behavior that may predict group membership as juvenile SO or NSO. Additionally, this study will investigate the association between sexual fantasy and sexual behavior and how that differs for these two groups (i.e., SO and NSO). 
Recent advances in technology raise a critical concern for the outcomes of this study. As previously mentioned, treatment and prevention efforts currently make many assumptions about the importance of sexual fantasy and sexual behavior in the etiology, assessment, and treatment of juvenile SOs. As reported in Chapter IV, evidence suggests that increased access to cell phones and high-speed broadband internet provides instant access to pornography, and communication with others (Schaeffer, 2019). Increased ease of access results in endless sexual stimuli, resulting in an equalizing effect that leads to most youth experiencing the same types of sexual fantasies. These findings underscore the need for these two constructs (i.e., sexual fantasies and sexual behavior) to be reexamined in light of significant changes in access to sexual stimuli. In the next chapter, hypotheses will be described and supported by key research findings. 
Chapter VIII: Development of Research Hypotheses

The preceding sections have provided an overview of the literature on the sexual fantasies and sexual behavior of juvenile offenders. The assumption that deviant sexual experiences and interests play an important role in sexual offending is one that is almost universally made by clinicians and is based on early research with extremely violent sexual offenders (SOs; Daleidan et al., 1998; Howitt, 2004). However, this assumption may lead to the overgeneralization of the importance of these constructs in sexual offending (Howitt, 2004). There is considerable research on the sexual fantasies and sexual behavior of juvenile SOs, yet there is a significant lack of research that compares this group to members of a control group on these constructs. To address this limitation in the existing research literature, sexual fantasy and sexual behavior will be measured in groups of adjudicated, juvenile SOs and nonsexual offenders (NSOs) as part of this study. The following section will discuss the development of research questions and hypotheses in support of the present study.

\section{Research Hypotheses}

Subgroup differences in deviant sexual fantasies within the last year. The first area of investigation is the sexual fantasies that juvenile offenders have experienced during the last 12-months. Historically, deviant sexual fantasies have been used as a treatment target for juvenile SOs (Abel \& Blanchard, 1974). According to the maintenance process of conditioning theory, behaviors (i.e., engaging in sexual fantasies) that are practiced and not reinforced with reward (i.e., orgasm) will become extinct. It would follow that deviant sexual fantasies that have been maintained most recently would 
be of primary interest to treatment professionals who are seeking to reduce the deviant content of SOs deviant sexual fantasies. To date, research has not distinguished recent fantasies from lifetime fantasies.

Hypothesis 1: It is hypothesized that SOs will report having more deviant sexual fantasies (i.e., global deviance) than NSOs within the last 12-months.

Using sexual fantasy to predict group membership. Next, to determine if deviant sexual fantasy is a significant predictor of offender group membership (i.e., SO or NSO), the second area of investigation explores the utility of Kaufman's (1998) Sexual Fantasy Questionnaire (SFQ) in predicting group membership (i.e., SO and NSO). Previous research has found that juvenile SOs have more Deviant and Non-Traditional sexual fantasies than NSOs (Daleiden et al., 1998). According to Seto and his colleagues (2010), the lack of research comparing juvenile SOs and NSOs leaves treatment professionals to rely on the theoretical literature to inform treatment decisions. As discussed previously, both the Social Deficits Model (Marshall et al., 1993) of sexual offending and the Conditioning Theory of Deviant Sexual Preference and Behavior (Laws \& Marshall, 1990) would suggest that deviant sexual fantasies should be a significant predictor of offender group membership (i.e., SO or NSO).

Hypothesis 2a: Deviant 12-month sexual fantasy (i.e., global deviance) will increase the predictive accuracy of Kaufman's Sexual Fantasy Questionnaire 
(1998) when predicting group membership after accounting for non-deviant 12month fantasies (i.e., global non-deviance).

Hypothesis 2b: Deviant lifetime sexual fantasy (i.e., global deviance) will increase the predictive accuracy of Kaufman's Sexual Fantasy Questionnaire (1998) when predicting group membership after accounting for non-deviant lifetime fantasies (i.e., global non-deviance).

Using sexual behavior to predict group membership. To determine if deviant sexual behavior is a significant predictor of offender group membership (i.e., SO or NSO), the third area of investigation explores the utility of Kaufman's (2014) Sexual History Form-Revised (SHF-R) in predicting group membership (i.e., SO and NSO). The limited previous research focusing on this topic has found group differences (Daleiden et al., 1998); however, a recent literature review found that investigation beyond between group comparisons of sexual behavior has not occurred. To increase the effectiveness of treatment, it is crucial to identify whether deviant sexual behavior, specifically, increases the predictive accuracy of group membership above and beyond traditional sexual behavior.

Previous research has found that juvenile SOs indicated engaging in fewer Typical/Consenting sexual behaviors (e.g., behaviors that are nondeviant - kissing, petting, and vaginal intercourse) and more Typical/Non-consenting (e.g., behaviors that are nondeviant - kissing, petting, and vaginal intercourse, but with a nonconsenting 
partner), Solitary Sexual Acts (e.g., masturbating alone, having sexual activity with animals, and looking at pornographic pictures), and Paraphilic sexual behaviors (e.g., masturbating in a public place, becoming sexually excited by watching fire) than juvenile NSOs (Daleiden et al., 1998). Additionally, Daleiden and his colleagues (1998) found that juvenile SOs and NSOs did not differ significantly in their experiences of Atypical/Consenting and Voyeuristic sexual behaviors.

Hypothesis 3: Deviant sexual behavior (i.e., atypical consensual (e.g., anal intercourse, paying a partner to have sex with you), aggressive/consensual (e.g., physically hurting, humiliating, or embarrassing your partner, threatening your partner), paraphilias, voyeurism (e.g., taking pictures or movies/videos of someone nude, secretly watching others have intercourse), and solitary sex acts will increase the predictive accuracy of Kaufman's (2014) Sexual History Form when predicting group membership after accounting for nondeviant sexual behavior (i.e., typical/consensual).

\section{Potential moderating effects of offender group on the relationship between} juvenile offenders' sexual fantasies and sexual behaviors. In an effort to establish a relationship between deviant sexual fantasy and deviant sexual behavior and therefore support the use of deviant sexual fantasy as a treatment target for this group, the third area of investigation looks at the potential moderating effect of group membership on the relationship between sexual fantasies and sexual behavior. The Conditioning Theory of 
Deviant Sexual Preference and Behavior suggests that the association of sexual fantasies and sexual pleasure increases the desire to act out those fantasies in the real world (Laws \& Marshall, 1990; Marshall \& Marshall, 2000). As discussed in Chapter 5, there is a greater understanding of the function of sexual fantasy, as it relates to sexual offending, in the adult population. Work by Gee and colleagues $(2003 ; 2004)$ with adult, male SOs, concluded that sexual fantasy serves as an offense script, which allows offenders to plan out the details of their offense, to achieve arousal. In addition, this research concluded that in a sample of adult, male SOs, all participants reported having offense-specific fantasies (Gee et al., 2004). These conclusions suggest that deviant sexual behaviors are informed by deviant sexual fantasies. While these connections have been drawn in research on adult SO populations, they have yet to be empirically tested with a sample of juvenile offenders. A series of hypotheses were tested to investigate the relationship between deviant sexual fantasies and the five different types of deviant sexual behavior (i.e., atypical consensual, aggressive consensual, voyeurism, paraphilias, solitary sex).

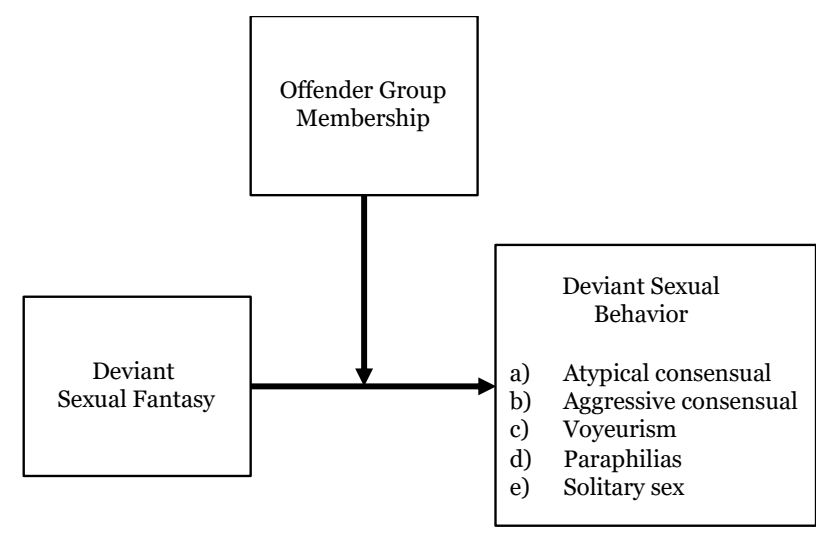

Hypothesis 4a: The relationship between deviant lifetime sexual fantasy and atypical consensual behavior will be moderated by group membership, such that, this relationship will be stronger for SOs than for NSOs. 
Hypothesis 4b: The relationship between deviant lifetime sexual fantasy and aggressive consensual behavior will be moderated by group membership, such that, this relationship will be stronger for SOs than for NSOs.

Hypothesis 4c: The relationship between deviant lifetime sexual fantasy and voyeuristic sexual behavior will be moderated by group membership, such that, this relationship will be stronger for SOs than for NSOs.

Hypothesis 4d: The relationship between deviant lifetime sexual fantasy and paraphilic behavior will be moderated by group membership, such that, this relationship will be stronger for SOs than for NSOs.

Hypothesis 4e: The relationship between deviant lifetime sexual fantasy and solitary sexual behavior will be moderated by group membership, such that, this relationship will be stronger for SOs than for NSOs.

The use of sexting by juvenile offenders. Finally, the fourth area of investigation sought to explore differences between the two offender groups (i.e., SOs and NSOs) in sexting. The phenomenon of sexting is a new construct of interest to the field of CSA. Research on sexting and adolescence is emerging, but research focused on its role in sexual offending is virtually nonexistent. It has been suggested that adolescents 
experience a significant part of their social and emotional development and sexual exploration through text messaging and social media (Cooper et al., 2016; O'Keefe \& Clarke-Pearson, 2011). At this point, it is unclear if sexting is a normalized behavior for adolescents, or if it is a behavior that juvenile SOs engage in more often than NSOs. For the purpose of exploring potential differences between these groups, there is no predicted direction.

Hypothesis 5: It is hypothesized that JSOs and JNSOs differ in the frequency of sexting.

The next chapter will provide information on the participants of the current study and the methodology used to test the hypotheses described above. 


\section{Chapter IX: Methods}

\section{Institutional Review Board Process}

The current study went through two Institutional Review Boards (IRB). First, the Oregon Youth Authority (OYA), the collaborating state agency from which study participants were accessed, reviewed the project. Once approved by OYA, the IRB at Portland State University reviewed, and approved, the project. Data used for this study was collected as part of a larger project investigating juvenile offenders' sexual fantasies, sexual behavior, modus operandi (i.e., sexual offenders only), and adverse childhood experiences (ACEs).

\section{Participants}

Participants for this study included 269 youth offenders recruited from four youth correctional facilities (YCF; MacLaren, Rogue Valley, Tillamook, Eastern Oregon) across the state of Oregon. Youth offenders range in age from 14 to 24 years old and were adjudicated for a variety of crimes (e.g., sexual offenses, person offenses, property offenses, substance use related offenses, robbery, homicide). In total, 87 sexual offenders (SO) and 181 nonsexual offenders (NSO) participated in the data collection, for a final sample of 268 participants $^{2}$. The SO sample had an average age of $19(S D=2.29)$ and the NSO sample had an average age of $18.16(S D=1.88)$, at the time of data collection.

\section{Participant Recruitment}

Prior to the informational session, describing the study to potential participants, staff were asked to identify any youth whose cognitive functioning or difficulty reading

\footnotetext{
${ }^{2}$ One youth did not complete the final packet indicating which offender group he belonged to.
} 
would make it challenging for them to participate in this study. No youth were identified by staff as needing to be excluded from participation, based on this criterion. In addition, youth were excluded if they have a severe mental health concern that would limit their ability to understand questionnaire items or respond with accuracy (e.g., severe depression, psychotic disorder). Two youth were excluded from participation due to concerns that the youth were at a high-risk of being triggered by the material.

Youth participants were recruited and completed measures while on their living units. All youth present at the time of data collection were invited to participate. Youth were provided with an informational session describing the purpose of the research study (i.e., to obtain data to better inform the treatment programs that they participate in, to contribute to our knowledge of how to best provide effective treatment to adolescent offenders, to inform healthy sexuality education for adolescents). Voluntary participation was emphasized, and youth were informed that their decision not to participate would, in no way, affect their standing or treatment with OYA. Youth were also told that they could withdraw from the study at any time without consequence. The fact that the data was collected anonymously was highlighted and participants were asked not to provide any identifying information on the surveys and to avoid providing any specific information about an unreported crime ${ }^{3}$. These reminders and cautions were also repeated at the time of data collection. A member of the research team read the assent form aloud, as youth participants followed along. Once youth had the opportunity to thoroughly review the assent form and ask any questions, individuals who did not wish to participate

\footnotetext{
${ }^{3}$ There is no question that asks participants to report details about unreported crimes in any of the surveys.
} 
were given the opportunity to opt out of the study. Across the four facilities, 14 youth made the decision not to participate and 3 youth chose not to complete the data collection session after they started.

\section{Procedure}

Consent for youth participation was obtained from the designated OYA representative at each facility. Youth who agreed to participate in the study completed the assent process. Once all assent forms had been collected, all data was obtained via paper and pencil questionnaires in groups of 20-25 youth, with each participant completing an individual survey packet. OYA staff, although present during data collection (as they are required to be in the room for supervision purposes), were not allowed to access participant surveys at any point during the data collection process. In fact, OYA staff were asked to refrain from interacting with the youth during the data collection (e.g., no answering youth's questions), unless required in response to youth's behavior. As an employee of OYA, I also did not interact with youth while they were completing packets. Although unlikely, this was an important step in ensuring that my involvement did not influence youth's responses. Dr. Kaufman provided enough staff to ensure that OYA youth were adequately supported in completing study measures.

Youth participants completed each of their study measures one at a time. The Sexual Fantasy Questionnaire (SFQ; Kaufman, 1998) was distributed first to each of the participating youth and instructions were provided to all participants at the same time. When youth completed the first measure, they were asked to raise their hand. Participants were asked to review their survey and check for any responses that they missed, and had 
intended to complete, then place their survey in an unmarked manila envelope on their workspace. Youth repeated this process as they completed each of the study measures, adding to the unmarked manila envelope which remained on the table in front of them until they completed all of the study measures. This process ensured youth confidentiality and, at the same time, kept each youth's packet of measures together for later analysis. Participants were offered a snack of their choice after completing the first survey. A member of the research team provided them with the next survey and reviewed the instructions with them, individually. This process was repeated for distribution of the third survey following completion of the second survey. Participants received surveys in the following order: Sexual Fantasy Questionnaire (Kaufman, 1998), Sexual History Form-Revised (SHF-R; Kaufman, 2014), and Demographics (Kaufman, 2014). Measures were distributed in this order to mitigate against priming by asking youth about their own experiences of sexual victimization prior to asking them about their sexual fantasies and behaviors. Data collection took approximately 1.5 hours, including distribution of the snack.

\section{Study Design}

The current study utilized a cross-sectional, non-experimental design. Specifically, quantitative survey data was collected from youth participants within OYA YCFs at only one time point. This study investigated the relationship between offender group, sexual fantasies, and sexual behavior.

\section{Descriptions and Measurement of Study Constructs}


Offender group. Participants were grouped based on identification by unit staff based on commitment offense and treatment needs.

Sexual fantasy questionnaire (Kaufman, 1998). The SFQ is a 143-item selfreport questionnaire that asks youth to indicate the frequency with which they have experienced a variety of sexual fantasies (e.g., How often do you fantasize about dressing in costumes during sexual activity?) and the context of which they experience these sexual fantasies (e.g., How often do you have fantasies during masturbation?). This questionnaire defines fantasy as "thoughts, feeling, or images that include sexual activities and feelings (Kaufman, 1998)." Each fantasy item is rated on a 7-point Likerttype scale from 0 (never) to 6 (two or more times a day). Each context item is rated on a 7-point Likert-type scale from 0 (never) to 6 (always). See Table 1 for a complete list of items.

A factor analysis of the SFQ in a sample of 350 adolescent sexual offenders resulted in two subscales (Daleiden et al., 1998). The first subscale, representing Global Deviance, included items such as whipping, beating, or torturing others, and fantasizing about paraphilias (Daleiden et al., 1998). The second subscale, representing Global NonDeviance, included items such as hugging, kissing, undressing, loving, and sex in romantic places (Daleiden et al., 1998).

Sexual history form-revised (Kaufman, 2014). The SHF-R is a 63-item selfreport questionnaire that asks youth to indicate the frequency with which they have engaged in a variety of sexual activities during their lifetime. Additionally, participants are asked to indicate how many times they engaged in each sexual activity with both 
consenting and nonconsenting partners (e.g., How often have you experienced having a consenting partner stroke or rub your genitals? How often have you experienced having a non-consenting partner stroke or rub your genitals?). Each item is rated on a 7-point Likert-type scale from 0 (Never in my life) to 6 (More than 50 times) for both consenting and non-consenting partners. See Table 2 for a complete list of items.

A factor analysis of the SHF in a sample of 350 youth who had committed sexual offenses and 315 undergraduate students resulted in eight subscales (Daleiden et al, 1998). Sexual activities with consenting partners were represented by three subscales (Daleiden et al, 1998). The Typical Sexual Acts subscale included items such as kissing, petting, and vaginal intercourse (Daleiden et al, 1998). The Atypical Sexual Acts subscale included items such as anal intercourse and paying money for sex (Daleiden et al, 1998). The Aggressive Sexual Acts subscale included items such as humiliating, frightening, and being physically hurt by one's partner (Daleiden et al, 1998). Sexual activities with non-consenting partners, or activities not requiring a partner, were represented by five subscales (Daleiden et al, 1998). The Typical, Non-consenting Acts subscale included items such as kissing, petting, and vaginal intercourse (Daleiden et al, 1998). The Voyeurism subscale included items such as watching others have intercourse and walking in on someone in the bathroom (Daleiden et al, 1998). The Paraphilias subscale included items such as rubbing against a stranger, cross-dressing, and being sexually excited by fire (Daleiden et al, 1998). Finally, the Solitary Sexual Acts subscale included items such as masturbating alone, having sexual activity with animals, and looking at pornographic pictures (Daleiden et al, 1998). Previous research using an earlier 
version of this measure demonstrated good to excellent internal consistency with several exceptions which were dependent on participant group (Daleiden et al, 1998).

The SHF-R (Kaufman, 2014) contains additional questions that are intended to measure sexting behaviors. Seven additional questions were added measuring consensual (e.g., Receiving a picture of someone's genitals/buttocks/breasts through text message/email/online chat from someone willingly) and nonconsensual sexting behaviors (e.g., Sending a naked picture of someone other than yourself, who was not willing to participate, through text message/email/online).

Demographics form (Kaufman, 2014). The Demographics Form asks questions about the participants regarding their age, race/ethnicity, educational history, history with abuse, offense history, and history of exposure to violence and family dysfunction. 


\section{Chapter X: Results}

A series of analyses were carried out to address the hypotheses outlined in Chapter VIII. This chapter details the manner in which those analyses were conducted, beginning with how data were screened for errors. Next, the demographics of the final sample are described. The results of preliminary analyses used to explore potential covariates and examine scale reliabilities are presented. Finally, the results for each inferential analysis conducted to address study hypotheses are summarized.

\section{Data Screening}

Prior to conducting analyses, data were screened for errors in data entry and missing values. Frequency distributions and range statistics were examined to identify any cases that were outside the range of plausible values on each variable of interest. For cases that had values out of the expected range, hard copies of the raw survey data were

double checked and corrected. One participant was excluded because he did not complete the entire series of surveys and it was not clear which offender group he belonged to.

\section{Sample}

The final sample consisted of 268 participants. Of these participants, 87 were sexual offenders (SO) and 181 were non-sexual offenders (NSO), with an average age of 19 and 18 years old, respectively, at the time of data collection. All participants were identified as male by the Oregon Youth Authority (OYA). Of the participants identified as SOs, $46(53 \%)$ self-reported being the victim of sexual abuse; whereas $23(13 \%)$ of the participants identified as NSOs self-reported this type of victimization in their history. 
Data on participant race and ethnicity were not collected. Youth of color are an underrepresented group among those who have committed sexual offenses. As of April 2020, there were 5 African American, 23 Hispanic, 6 Native American, 1 Asian, 79 White, and 1 other/unreported youth, who had committed sexual offenses, in OYA's youth correctional facilities (M. Greenwald, OYA Research Manager, personal communication, April 21, 2020). OYA expressed concern that youth could be identified based on offender grouping if this information is collected. However, as of July 2017 , when data collection began, OYA reported that $52 \%$ of all youth in close custody were Caucasian, 26\% were Hispanic, 14\% were African American, 5\% were Native American, $2 \%$ were other/unreported, and 1\% were Asian (OYA Quick Facts, 2017). According to Kaiser Family Foundation State Health Facts for 2017, the population of children, ages 017, in Oregon had the following ethnic/racial breakdown: $63 \%$ white, $22 \%$ Hispanic, $2 \%$ Black, 4\% Asian/Native Hawaiian and Pacific Islander, 1\% American Indian/Alaska Native, and 7\% multiple races (Kaiser Family Foundation, 2020).

\section{Preliminary Analyses}

Covariates of interest were investigated for inclusion in inferential analyses. Based on previous research, the following covariates were investigated: 1) history of sexual abuse victimization and 2) social desirability (Daleiden et al., 1998; Kaufman et al., 1996; \& Kaufman et al., 1998; Table 3). History of sexual abuse was not significantly correlated with any variables of interest and was not included in further analyses. Social desirability, as measure by the shortened Marlowe Crowne Social Desirability Scale 
(Reynold, 1982), was significantly correlated with several variables of interest, but was not correlated with offender group (Table 3).

To examine the reliability of the various subscales, Chronbach's alpha coefficients were calculated using the final sample. Chronbach's alpha coefficients ranged from .65-.98, with the 10 of 13 subscales having coefficients over .81 (Table 4 ). There is a direct relationship between reliability and the number of items that comprise the subscale. The three subscales that had Chronbach's alpha coefficients below .81 (i.e., Atypical/Consensual=.65, Voyeurism=.65, Solitary Sex Acts=.72) were all comprised of only four items. The Spearman-Brown Formula estimates how much a scale's reliability would improve with additional high-quality items. If the Atypical Consensual and Voyeurism subscales were doubled in length, 8-items, the Chronbach's alpha would increase to .79. If the Solitary Sex subscale was doubled in length, 8-items, the Chronback's alpha would increase to .84 . The results of the Spearman-Brown Formula, and the understanding that significant results should be interpreted with caution, lead to the conclusions that the reliability of these subscale was deemed appropriate for this, an initial study in this area. Additionally, the shortened form of the Marlowe Crowne Social Desirability Scale (13-items) had low reliability, .55. Further exploration identified threeitems that were contributing to the low reliability of this scale (i.e., I sometimes try to get even rather than forgive, I have never been irked when people expressed ideas very different from my own, and I have never deliberately said something that hurt someone's feelings). Removal of these three items resulted in a 10-item scale with a reliability of 
.70 , which is considered acceptable for research. The 10-item version of this scale was used as a covariate in Hypotheses 2 and 3.

\section{Inferential Analyses}

Several analyses included two different measures of deviant sexual fantasies.

First, deviant sexual fantasies that have occurred in the participant's lifetime is identified, and going forward is referred to as, "deviant lifetime sexual fantasies." Second, deviant sexual fantasies that participants report experiencing in the last 12 months, is denoted as “deviant 12-month sexual fantasies" for the remainder of this document.

Offender group inclusion was determined based on identification made by OYA using a combination of the individual's commitment offense and their assessed treatment need. Consideration was also given to the possibility that some youth in the NSO sample may have committed sexual offenses and not been adjudicated. Therefore, in addition to grouping offenders based on commitment type and treatment need, each analysis was also run a second time using offender groupings based on participant's self-report responses to nonconsensual items in the Sexual History Form-Revised (SHF-R; Kaufman, 2014). Using this grouping criterion, 24 additional youth self-identified as SOs, resulting in 111 sexual offenders (SOs) and 157 non-sexual offenders (NSO). Using this dual grouping criteria did not change substantive study findings. Therefore, those results will only be reported for analyses using offender groupings determined by OYA.

\section{Subgroup difference in deviant 12-month sexual fantasies.}


Hypothesis 1: It is hypothesized that SOs will report having more deviant sexual fantasies (i.e., global deviance) than NSOs within the last 12-months.

The independent variable for this analysis was offender group with two levels SO $(n=87)$ and NSO $(n=179)$. The dependent variable was deviant 12-month sexual fantasies. Outliers were identified through inspection of boxplot and distance, in standard deviations, from the mean. In total, 18 cases were identified as outliers. Deviant 12month sexual fantasies for each level of offender group were not normally distributed, as assed by Shapiro-Wilk’s test $(p<.001)$ and Normal Q-Q Plots. A square root transformation was applied to the dependent variable in an attempt to resolve the concern of outliers and the violation of the assumption of normality. This transformation improved, but did not resolve, these concerns. Independent-samples t-tests are robust against violations of this assumption. This violation becomes less problematic when skew is in the same direction for both groups, which was the case here. Inferential tests were run with and without this transformation applied and with and without outliers and substantively similar conclusions were reached. Inferential statistics resulting from tests including the variables without transformation and with the inclusion of outliers are reported to facilitate interpretation. There was homogeneity of variances, as assessed by Levene's test for equality of variance $(p=.08)$. A significant difference was not found between SOs $(M=.44, S D=.78)$ and NSOs $(M=.63, S D=.96), 95 \%$ CI $[-.04, .43]$, $t(246)=1.66, p=.10$. 


\section{Using sexual fantasy to predict offender group membership. Prior to}

conducting inferential analyses, subgroup differences on each of the sexual fantasy subscales were assessed using a series of independent samples t-tests. The independent variable for all analyses was offender group. Dependent variables included each of the sexual fantasy subscales (i.e., nondeviant 12-month sexual fantasies, deviant 12-month sexual fantasies, nondeviant lifetime sexual fantasies, and deviant lifetime sexual fantasies). Means and standard deviations for each subscale are presented in Table 5. Only one sexual fantasy subscale had significant group differences. Specifically, nondeviant 12-month sexual fantasies were reported with greater frequency by NSOs $(M$ $=.07, S D=.04)$ than by SOs $(M=.06, S D=.03), 95 \%$ CI $[.00, .02], t(264)=2.74, p<$ .01 .

Two three-step, hierarchical, logistic regressions were conducted to assess the added utility of deviant sexual fantasies in predicting group membership using nondeviant and deviant sexual fantasies. The first analysis explored 12-month sexual fantasies (Hypothesis 2a). The second analysis explored lifetime sexual fantasies (Hypothesis 2b). For both models, independent variables were entered in the following order: Step 1: covariate (Social Desirability); Step 2: non-deviant sexual fantasies; Step 3: deviant sexual fantasies. Linearity of the continuous variables with respect to the logit of the dependent variable was assessed via the Box-Tidwell (1962) procedure. A Bonferroni correction was applied using both terms in the model resulting in statistical significance being accepted when $p<.03$ (Tabachnick \& Fidell, 2014). Based on this assessment all 
continuous independent variables were found to be linearly related to the logit of the dependent variable. No outliers were found.

Hypothesis 2a: Deviant 12-month sexual fantasy (i.e., global deviance) will increase the predictive accuracy of Kaufman's Sexual Fantasy Questionnaire (1998) when predicting group membership after accounting for nondeviant 12month fantasies (i.e., global non-deviance).

After controlling for the social desirability and nondeviant 12-month sexual fantasies, deviant 12-month sexual fantasies were not a significant predictor of offender group membership. The final model was not statistically significant, $X^{2}(3)=3.86, p=$ .28. Model fit was good, $X^{2}(8)=5.40, p=.71$, as determined by Hosmer and Lemeshow Test for goodness of fit. The model explained $2 \%$ of the variance in offender group, as determined by Nagelkerke's $R^{2}$, and correctly classified $66.4 \%$ of cases. Model comparisons are presented in Table 6. Sensitivity (i.e., \% of correct SO predictions) was $1.2 \%$, specificity (i.e., $\%$ of correct NSO predictions) was $100 \%$, positive predictive value (i.e., \% correct SO predictions compared to total number of predicted SOs) was $100 \%$, and negative predictive value (i.e., \% correct NSO predictions compared to total number of predicted NSOs) was $66.3 \%$.

Hypothesis 2b: Deviant lifetime sexual fantasy (i.e., global deviance) will increase the predictive accuracy of Kaufman's Sexual Fantasy Questionnaire (1998) 
when predicting group membership after accounting for nondeviant lifetime fantasies (i.e., global non-deviance).

After controlling for the social desirability and nondeviant lifetime sexual fantasies, deviant lifetime sexual fantasies were not a significant predictor of offender group membership. The final model was not statistically significant, $X^{2}(3)=6.91, p=$ .08 . Model fit was good, $X^{2}(8)=9.81, p=.28$, as determined by Hosmer and Lemeshow Test for goodness of fit. The model explained $4 \%$ of the variance in offender group, as determined by Nagelkerke's $R^{2}$, and correctly classified $66 \%$ of cases. Model comparisons are presented in Table 7. Sensitivity (i.e., \% of correct SO predictions) was $1.2 \%$, specificity (i.e., $\%$ of correct NSO predictions) was $99.4 \%$, positive predictive value (i.e., \% correct SO predictions compared to total number of predicted SOs) was $50 \%$, and negative predictive value (i.e., \% correct NSO predictions compared to total number of predicted NSOs) was $66.1 \%$.

\section{Using sexual behavior to predict offender group membership. Prior to}

conducting inferential test, subgroup differences on each of the sexual behavior subscales were assessed using a series of independent samples t-tests. The independent variable for all analyses was offender group. Dependent variables included each of the sexual behavior subscales (i.e., typical consensual, atypical consensual, aggressive consensual, voyeurism, paraphilias, and solitary sex). Means and standard deviations for each subscale are presented in Table 5. Analyses for three of the six subscales resulted in significant differences. Specifically, typical consensual behaviors were reported with 
greater frequency by NSOs $(M=.33, S D=.15)$ than by SOs $(M=.26, S D=.16), 95 \% \mathrm{CI}$ $[.03, .11], t(262)=3.34, p<.01$. Voyeurism was also reported with greater frequency by NSOs $(M=.20, S D=.25)$ than by SOs $(M=.12, S D=.21), 95 \%$ CI $[.02, .14], t(263)=$ $2.59, p<.05$. Alternatively, solitary sex was reported with greater frequency by SOs $(M$ $=.76, S D=.30)$ than by NSOs $(M=.64, S D=.38), 95 \%$ CI $[-.21,-.03], t(264)=-2.57, p$ $<.05$.

A three-step, hierarchical, logistic regression was conducted to assess the added utility of deviant sexual behaviors in predicting group membership using sexual behavior. Independent variables were entered in the following order: Step 1: covariate (Length of Time in Sex Offense Specific Treatment); Step 2: nondeviant sexual behavior (Typical Consensual); Step 3: deviant sexual behavior (Aggressive Consensual, Typical Consensual, Voyeurism, Paraphilias, and Solitary Sex). Linearity of the continuous variables with respect to the logit of the dependent variable was assessed via the BoxTidwell (1962) procedure. A Bonferroni correction was applied using all seven terms in the model resulting in statistical significance being accepted when $p<.007$ (Tabachnick \& Fidell, 2014). Based on this assessment all continuous independent variables were found to be linearly related to the logit of the dependent variable. No outliers were found.

Hypothesis 3: Deviant sexual behavior (i.e., atypical consensual, aggressive/consensual, paraphilias, solitary sex, and voyeurism) will increase the predictive accuracy of Kaufman's (2014) Sexual History Form when predicting 
group membership after accounting for nondeviant sexual behavior (i.e., typical/consensual).

After controlling for the social desirability and nondeviant sexual behavior, solitary sexual behavior and voyeurism were significant predictors of offender group membership. The final model was statistically significant, $X^{2}(7)=39.811, p<.01$. Model fit was good, $X^{2}(8)=7.78, p=.46$, as determined by Hosmer and Lemeshow Test for goodness of fit. The model explained $22 \%$ of the variance in offender group, as determined by Nagelkerke's $R^{2}$, and correctly classified $72.6 \%$ of cases. Model comparisons are presented in Table 8 . Sensitivity (i.e., \% of correct SO predictions) was $38 \%$, specificity (i.e., $\%$ of correct NSO predictions) was $90.3 \%$, positive predictive value (i.e., \% correct SO predictions compared to total number of predicted SOs) was $66.7 \%$, and negative predictive value (i.e., \% correct NSO predictions compared to total number of predicted NSOs) was $74 \%$.

Of the five variables measuring deviant sexual behavior, only two were significant, after accounting for social desirability and nondeviant sexual behavior: solitary sexual acts and voyeurism (as shown in Table 9). Increased reporting of solitary sexual behavior was associated with an increased likelihood of being a SO, while increased reporting of voyeurism was associated with a reduction in the likelihood of being an SO.

To better understand the significant finding related to solitary sex and voyeurism, follow-up analyses were conducted. A series of $t$-tests were run to investigate group 
differences at the item level. After applying a Bonferroni correction to adjust the significance level to account for a larger number of tests, a significance level of .01 was used as the criterion for significance. First, significant differences were found for two of the four items on the solitary sexual acts subscale. Sexual contact with an animal was reported with greater frequency by SOs $(M=.31, S D=.67)$ than NSOs $(M=.06, S D=$ $.50), 95 \%$ CI $[-.40,-.11], t(263)=-3.43, p<.01$. Masturbating alone was reported with greater frequency by SOs $(M=4.49, S D=2.17)$ than by NSOs $(M=3.72, S D=2.35)$, $95 \%$ CI $[-1.37,-.18], t(263)=-2.55, p<.01$. Significant differences between SOs and NSOs were found on one of the four items (i.e., taking pictures or movies/videos of someone naked) on the voyeurism subscale, 95\% CI [.26, 1.18], $t(263)=3.10, p<.01$, with NSOs reporting more $(M=1.61, S D=1.89)$ voyeurism than $\mathrm{SOs}(M=.86, S D=$ 1.53). The other three items did not reveal significant differences.

Potential moderating effects of offender group on the relationship between sexual fantasy and sexual behavior. A series of moderated multiple regressions were conducted to examine whether offender group membership moderates the relationship between deviant sexual fantasies and deviant sexual behaviors. Each of the following sexual behaviors served as a dependent variable in respective analyses: Atypical Consensual (Hypothesis 3a), Aggressive Consensual (Hypothesis 3b), Voyuerism (Hypothesis 3c), Paraphilias, (Hypothesis 3d), and Solitary Sex (Hypothesis 3e). The independent variables, for all analyses were deviant sexual fantasies and offender group membership. Each sexual behavior was examined in relation to deviant lifetime (Sub Hypothesis 1) and 12-month sexual fantasies (Sub Hypothesis 2). The moderator 
relationship was determined by the significance of the interaction of deviant sexual fantasies and offender group membership.

For all analyses, linearity was established by visual inspection of a scatterplot and there was no evidence of multicollinearity, as evidenced by no tolerance values less than 0.54. Unusual points were identified through inspection of outliers, leverage, and influence. For all analyses, these points were not removed from analysis, as substantively similar conclusions were reached with and without the unusual points included in the analysis. The assumption of homoscedasticity was violated, as determined by visual inspection of the studentized residuals plotted against the predicted values for juvenile sexual and non-sexual offenders. A square root transformation of the dependent variable was applied and did not resolve this violation, nor did it change the substantive conclusion that was reached. All analyses indicated a pattern of heteroscedasticity that resulted in estimates of standard errors that are too small, producing confidence intervals that are too narrow. This makes hypothesis testing invalid for these regression coefficients (Darlington \& Hayes, 2017). To correct for the violation of homoscedasticity, analyses were conducted using the RLM Macro (Darlington \& Hayes, 2017). This macro uses heteroscedasticity-consistent standard error estimators for the regression coefficients (Darlington \& Hayes, 2017). All results reported are those that resulted from analyses using the RLM macro to correct for homoscedasticity.

In addition, studentized residuals were not normally distributed, as assessed by Normal Q-Q plots. A square root transformation of the dependent variable was applied and did not resolve this violation, nor did it change the substantive conclusion that was 
reached. Therefore, the original variables were retained, accepting a loss of power for violating the assumption of normality.

Hypothesis 4a1: The relationship between deviant lifetime sexual fantasies and atypical consensual sexual behavior will be moderated by group membership, such that, this relationship will be stronger for SOs than for NSOs.

A moderated multiple regression was run to assess the statistical significance of the interaction term between lifetime deviant sexual fantasies and offender group membership as it relates to atypical consensual behaviors. Group membership did not moderate the effect of lifetime deviant fantasies on atypical consensual sexual behavior, as evidenced by an increase in total variation explained of $0.3 \%$, which was not statistically significant, $F(1,259)=.74, \mathrm{p}=.39$.

Hypothesis 4a2: The relationship between deviant 12-month sexual fantasies and atypical consensual sexual behavior will be moderated by group membership, such that, this relationship will be stronger for SOs than for NSOs.

A moderated multiple regression was run to assess the statistical significance of the interaction term between 12-month deviant sexual fantasies and offender group membership as it relates to atypical sexual behaviors. Group membership did not moderate the effect of 12-month deviant fantasies on atypical consensual sexual behavior, 
as evidenced by an increase in total variation explained of $0.2 \%$, which was not statistically significant, $F(1,261)=.90, \mathrm{p}=.34$.

Hypothesis 4b1: The relationship between deviant lifetime sexual fantasies and aggressive consensual sexual behavior will be moderated by group membership, such that, this relationship will be stronger for SOs than for NSOs.

A moderated multiple regression was run to assess the statistical significance of the interaction term between lifetime deviant sexual fantasies and offender group membership as it relates to aggressive consensual behaviors. Offender group membership did not moderate the effect of lifetime deviant sexual fantasies on aggressive consensual sexual behaviors, as evidenced by an increase in total variation explained of $3 \%$, which was not statistically significant, $F(1,258)=1.87, \mathrm{p}=.17$.

Hypothesis 4b2: The relationship between deviant 12-month sexual fantasies and aggressive consensual sexual behavior will be moderated by group membership, such that, this relationship will be stronger for SOs than for NSOs.

A moderated multiple regression was run to assess the statistical significance of the interaction term between 12-month deviant sexual fantasies and offender group membership as it related to aggressive consensual behaviors. Offender group membership did not moderate the effect of 12-month deviant sexual fantasies on aggressive 
consensual sexual behaviors, as evidenced by an increase in total variation explained of $2.7 \%$, which was not statistically significant, $F(1,260)=3.72, \mathrm{p}=.06$.

While this result is not statistically significant at a $p<.05$ level, it is trending toward significance. Therefore, simple slopes analyses were conducted to better understand the nature of this relationship. Simple slopes analysis revealed that there was a positive linear relationship (14.157 \pm 1.68$)$ between aggressive consensual sexual behavior and deviant 12-month sexual fantasies for SOs. Additionally, simple slopes analysis revealed that there was a positive linear relationship $(7.82 \pm .95)$ between aggressive consensual sexual behavior and deviant 12-month sexual fantasies for NSOs. Figure 1 provides a graphical representation of these analyses.

Hypothesis 4c1: The relationship between deviant lifetime sexual fantasies and voyeuristic sexual behavior will be moderated by group membership, such that, this relationship will be stronger for SOs than for NSOs.

A moderated multiple regression was run to assess the statistical significance of the interaction term between lifetime deviant sexual fantasies and offender group membership as it relates to voyeuristic behavior. Group membership did not moderate the effect of lifetime deviant fantasies on voyeuristic behavior, as evidenced by an increase in total variation explained of $0.9 \%$, which was not statistically significant, $F(1,256)=.57$, $\mathrm{p}=.45$. 
Hypothesis 4c2: The relationship between deviant 12-month sexual fantasies and voyeuristic sexual behavior will be moderated by group membership, such that, this relationship will be stronger for SOs than for NSOs.

A moderated multiple regression was run to assess the statistical significance of the interaction term between 12-month deviant sexual fantasies and offender group membership as it relates to voyeuristic behavior. Group membership did not moderate the effect of 12-month deviant fantasies on voyeuristic behavior, as evidenced by an increase in total variation explained of $0.9 \%$, which was not statistically significant, $F(1,259)=$ $.69, \mathrm{p}=.41$

Hypothesis 4d1: The relationship between deviant lifetime sexual fantasies and paraphilic sexual behavior will be moderated by group membership, such that, this relationship will be stronger for SOs than for NSOs.

A moderated multiple regression was run to assess the statistical significance of the interaction term between lifetime deviant sexual fantasies and offender group membership as it relates to paraphilic behaviors. Offender group membership did not moderate the effect of lifetime deviant sexual fantasies on paraphilic behaviors, as evidenced by an increase in total variation explained of $8.1 \%$, which was not statistically significant, $F(1,255)=1.28, \mathrm{p}=.26$. 
Hypothesis 4d2: The relationship between deviant 12-month sexual fantasies and paraphilic sexual behavior will be moderated by group membership, such that, this relationship will be stronger for SOs than for NSOs.

A moderated multiple regression was run to assess the statistical significance of the interaction term between 12-month deviant sexual fantasies and offender group membership as it relates to paraphilic behavior. Offender group membership did not moderate the effect of 12-month deviant sexual fantasies on paraphilic behaviors, as evidenced by an increase in total variation explained of $9.1 \%$, which was not statistically significant, $F(1,258)=1.19, \mathrm{p}=.28$.

Hypothesis 4e1: The relationship between deviant lifetime sexual fantasies and solitary sexual behavior will be moderated by group membership, such that, this relationship will be stronger for SOs than for NSOs.

A moderated multiple regression was run to assess the statistical significance of the interaction term between lifetime deviant sexual fantasies and offender group membership as it relates to solitary sexual behavior. Group membership did not moderate the effect of lifetime deviant fantasies on solitary sexual behavior, as evidenced by an increase in total variation explained of $0.3 \%$, which was not statistically significant, $F(1$, $257)=1.03, \mathrm{p}=.31$. 
Hypothesis 4e2: The relationship between deviant 12-month sexual fantasies and solitary sexual behavior will be moderated by group membership, such that, this relationship will be stronger for SOs than for NSOs.

A moderated multiple regression was run to assess the statistical significance of the interaction term between 12-month deviant sexual fantasies and offender group membership as it relates to solitary sexual behavior. Group membership did not moderate the effect of lifetime deviant fantasies on solitary sexual behavior, as evidenced by an increase in total variation explained of $0.1 \%$, which was not statistically significant, $F(1$, $260)=.38, \mathrm{p}=.54$

The use of sexting. Two one-way multivariate analysis of variance (MANOVA), were run to determine the effect of offender group on sexting behaviors.

Hypothesis 5a: SOs and NSOs differ in the frequency of their use of consensual sexting.

The independent variable was offender group with two levels $-\mathrm{SO}(\mathrm{n}=86)$ and NSO $(n=178)$. The dependent variables were seven items, listed in Table 2 , measuring consensual sexual behavior. Preliminary assumption checking revealed that four of the seven dependent variables were not normally distributed, as assessed by normal Q-Q plots. A square root transformation was applied to address the significant positive skew 
on these variables. The square root transformation addressed univariate outliers identified through boxplots. Inferential tests were run with and without this transformation applied and conclusions drawn from inferential statistics were the same. Inferential statistics resulting from tests including the variables without transformation are reported to ease interpretation.

Two multivariate outliers were identified using Mahalanobis distance $(p>.001)$. Outliers were retained for final analysis because their removal did not change the conclusions drawn from inferential statistics. Multicollinearity resulted in the removal of two items: 1) Sending a sexual video of someone's genitals/buttocks/breasts through text message/email/social media and 2) Sending messages with sexual content through text message/email/social media (Table 10). Scatterplots identified non-linear relationships that were not corrected following transformation. Analysis proceeded with the acceptance of a loss of power to detect significant differences. Finally, there was homogeneity of variance-covariance matrices, as assessed by Box's M test $(p=.001)$.

The differences between the offender groups on the combined dependent variables was statistically significant, $F(5,264)=3.02, p<.05$; Pillai's $=.95 ;$ partial $\eta^{2}=$ .06. Examination of between subjects effects indicated a statistically significant difference $\left(F(1,264)=8.96, p<.01\right.$; partial $\left.\eta^{2}=.03\right)$ between SOs and NSOs on 'Receiving a picture of someone's genitals/buttocks/breasts through text message/email/social media', using a Bonferroni adjusted level of .01. No other significant between subject effects were found. Means and standard deviation of each group are available in Table 11. 


\section{Hypothesis 5b: SOs and NSOs differ in the frequency of their use of} nonconsensual sexting.

The independent variable was offender group with two levels $-\mathrm{SO}(\mathrm{n}=84)$ and NSO $(n=175)$. The dependent variables were seven items, listed in Table 2 , measuring nonconsensual sexual behavior. Preliminary assumption checking revealed that all seven dependent variables were not normally distributed, as assessed by normal Q-Q plots. A square root transformation was applied to address the significant positive skew on these variables. The square root transformation did not reconcile univariate outliers identified through boxplots. Upon investigation of the univariate outliers, it was found that a vast majority of respondents $(n=240-256$ out of 268) responded with 0 'never in my life' on each of the seven items. See table 12 for item frequencies. Multicollinearity resulted in the removal of three items: 1) Sending a picture of your penis or buttocks to someone through text message/email/social media; 2) Sending sexual video of you and another person through text message/email/social media; and 3) Sending messages with sexual content through text message/email/social media (Table 13). Removal of three items due to multicollinearity and 14 multivariate outliers identified using Mahalanobis distance ( $p$ $>.001)$, resulted in group means of 0 on each of the remaining dependent variables. Inferential tests were not carried out as a result of the lack of variance in participant responses on these items. 


\section{Chapter XI: Discussion}

For decades, juvenile sexual offender prevention and treatment efforts have focused on the role of sexual fantasy and sexual behavior in the perpetration of offending behavior (Abel \& Blanchard, 1974). Despite the historical reliance on reported sexual behavior and sexual fantasies for treatment and prevention planning, there has been virtually no research to support assumptions that particular sexual fantasies and/or sexual behavior are unique to juvenile sexual offenders (SOs) and predictive of the need for sex offender specific treatment. The purpose of this research was to investigate differences in sexual fantasy and sexual behavior between juvenile sexual and non-sexual offenders.

This study had two primary goals. First, it broadly explored differences between juvenile SOs and non-sexual offenders' (NSOs) sexual fantasies and behavior. This was achieved by investigating offender group differences in deviant 12-month sexual fantasies (i.e., Hypothesis 1), the predictive utility of deviant sexual fantasies and behavior after accounting for nondeviant sexual fantasies and behavior (i.e., Hypotheses 2 and 3), and the potential for offender group to moderate the relationship between deviant sexual fantasies and deviant sexual behavior (i.e., Hypothesis 4). Second, this study explored differences in the use of sexting between these two groups (i.e., Hypothesis 5). Findings indicated that SOs did not significantly differ from NSOs on any measures related to sexual fantasies. Significant differences were, however, found related to sexual behaviors and sexting.

Before proceeding, it is important to note that this study encountered a significant challenge related to examining the question of whether SO's and NSOs differ in their 
sexual fantasies and/or their past sexual behaviors. Research conducted in the late 1990s indicated significant differences between SO's and NSO's deviant sexual fantasies and sexual behaviors. These findings had a profound impact on the treatment targets that were identified for sex offense specific clinical treatment protocols. As previously mentioned, these findings led clinical treatment facilities to assume that juvenile offenders reporting past deviant fantasies and/or deviant behavior required a tailored treatment regimen to address these concerns. Of note, is the fact that these studies lacked appropriate control groups, calling into question whether these past sexual fantasies and sexual behaviors truly distinguished them from NSOs. Moreover, it has been over two decades since this study was published and research demonstrates that what is considered "deviant" has changed over time (Langevin, 1998). For this study, the challenge is in the recognition that a true lack of differences between SOs and NSOs on measures of sexual fantasy or sexual behavior are not a possible outcome. Given that statistical rules prohibit interpreting nonsignificant differences as a true lack of group differences on a particular dimension, it is impossible to fully test both sides of this question. In fact, the literature suggests conclusions that can be drawn from nonsignificant findings are limited to, "assuming the true effect size in the population is zero, the observed effect size was not sufficiently different from zero to reject the null hypothesis without, in the long run, being wrong more often than a desired error rate (Harms \& Lakens, 2018 p. 383).” Therefore, it cannot be concluded that because no statistical difference was found, that there is a truly no difference in the population on any particular variable. 
The field is left in a conundrum where null results (i.e., findings that are not significant) are dismissed as meaningless findings (de Graaf \& Sack, 2011). At the same time, there is: current unfettered internet access to virtually any type of sexual behavior (Klein, 2014); evidence that adolescents and adults access a wide range of sexual material on the internet (Klein, 2014); and at least some of this material would currently or would have in the past been considered "deviant" (Klein, 2014). Taken together, these factors blur the current definition of what is "deviant" sexual behavior, intimates that the definition of "deviant" has likely changed dramatically over the past 20 years, and suggests that there may be more in common, than different about SO and NSO's sexual fantasies and behavior. Given these factors it becomes critical to be able to fully explore how SOs and NSOs do or do not differ in their sexual fantasies and sexual behaviors. Until we can do so, the question will remain as to whether this long-standing approach to treating SOs is empirically indicated. Since treatment resources are limited, particularly in juvenile correctional settings, this may also mean that these same youth may not have an opportunity to access many empirically supported and promising skills-based training areas that can positively impact their future reintegration into society and success in the community.

Returning to the issue of prohibitions against interpreting statistically null findings, it is notable that some authors have called for a change to this practice. For example, Earp (2017) urged the publication of null results for the purposes of creating a comprehensive body of literature. He argues that null results are not meaningless, but rather help to construct a true picture of a phenomenon. Therefore, while acknowledging 
that it is not common practice to interpret null findings, this chapter explores potential underlying reasons for the lack of differences between juvenile SOs and NSOs on measures of sexual fantasy and sexual behavior with the goal of beginning to build evidence for the possibility that a lack of statistical differences in this study may, in fact, reflect the fact that SOs and NSOs may not differ on many of these domains.

The remainder of this chapter will provide a detailed discussion of findings and recommended future directions, as they relate to each of the primary study questions. This discussion will include an examination of study limitations, as well. Finally, the chapter concludes with suggested implications for theory related to juvenile sexual offending and juvenile SO treatment, prevention efforts, and policy development.

\section{Sexual Fantasy and Sexual Behavior}

This section will provide a discussion of the findings and future directions addressing the first goal of this study, exploring differences in sexual fantasy and sexual behavior between juvenile SOs and NSOs. One significant finding emerged related to sexual fantasy and sexual behavior. The third research hypothesis, which suggested that deviant sexual behavior would add predictive utility, after accounting for nondeviant sexual behavior, when predicting group membership was supported. After controlling for social desirability and nondeviant sexual behavior, solitary sexual behavior and voyeurism were significant predictors of offender group (i.e., SO or NSO; Hypothesis 3). Specifically, increased reporting of solitary sexual behavior was associated with an increased likelihood of being an SO. This finding is supported by previous research conducted by Daleidan and his colleagues (1998) and by the Social Deficits Model 
(Marshall et al., 1993) and the Conditioning Theory of Deviant Sexual Preference and Behavior (Laws \& Marshall, 1990). The solitary sexual acts subscale is comprised of four items: sexual contact with animals, masturbating alone, looking at erotic magazines (e.g., Playboy, Hustler), and looking at pictures or movies/videos of someone naked. Further exploration of the items on this subscale revealed significant group differences for sexual contact with animals and masturbating alone. This supports the idea previously proposed in the literature that many SOs have limited social skills or are socially immature and as a result are unable to form intimate relationships with appropriate age peers and, instead, rely on solo masturbation to achieve sexual arousal and sexual gratification (Marshall et al., 1993). The second item that revealed significant differences, sexual contact with animals, has also been linked to difficulties with relating to humans and the ability to bypass negotiations for sex (Hensley et al., 2006).

Additionally, increased reporting of voyeurism was associated with a reduction in the likelihood of being an SO. Previous research found elevated levels of voyeurism in both SOs and NSOs, when compared to a community sample (SOs and NSOs did not differ from each other; Daleidan et al., 1998). The voyeurism subscale is comprised of four items: taking pictures or movies/videos of someone nude, taking pictures or movies/videos of people engaging in sexual activity, secretly watching others have intercourse, and flashing or exposing your genitals. Closer inspection of the data indicated that only one of these items was statistically different between groups (i.e., taking pictures or movies/videos of someone nude). This item is written in a way that does not indicate secrecy or deviancy and may have been confused for a consensual act 
and/or a part of sexting behavior that has become a normalized phenomenon among teens (Cooper et al, 2016). This finding is also supported by preliminary analyses that revealed subgroup differences which indicated that NSOs reported a greater frequency of typical consensual sexual behaviors than SOs (Table 5).

Overall, results were nonsignificant for all analyses related to the measure of sexual fantasy. Research focused on sexuality is met with many challenges, as sexuality is a very personal and private topic. Moreover, additional challenges have been associated with the study of deviance. Deviance, by definition, means "rare," making it a measurement and detection challenge (Langevin, 1998). Data in this study underscored this dilemma. Low group means reflected a lack of variance, making group differences even more difficult to detect across all analyses, but particularly those exploring sexual fantasy (see Table 5).

The experience of incarceration may have also had an impact on study findings. First, being incarcerated greatly limits the stimuli that most adolescents can access on a daily basis. Living unit staff monitor and significantly limit the entertainment that youth can access (e.g., TV shows, movies, cell phones). Moreover, in the correctional facilities, youth are prohibited from possessing or looking at pornographic material. Finally, their interactions with others are severely limited to the other youth in their facility, mostly their living unit, and facility staff. As such, they are likely to have much fewer externally prompted sexual fantasies, which limits the ability to develop new and varied sexual fantasies. Additionally, it is possible that many of the youth in this sample have few to no deviant sexual fantasies and their sexual offense was one of opportunity or impulse. To 
the extent that deviant fantasies have a low base-rate to begin with, it is not surprising to see very low rates in these incarcerated samples.

In addition, youth who are residing in youth correctional facilities at the Oregon Youth Authority (OYA) live in dorm like settings with up to 24 other young men. It is normal adolescent behavior to share a significant amount of their sexual experiences with their peers (Epstein \& Ward, 2008). This exchange of information by peers may affect the type and frequency with which participants experience sexual fantasies, creating an “equalizing" affect. It is also possible that youth's communication about fantasies to others in the facility may reduce the potential for group differences. For example, if a youth committed to the facility for a sexual offense tells an NSO dormmate about a fantasy, it may trigger a new, similar fantasy in the NSO youth. This process may artificially reduce the number of true differences in sexual fantasies that exist between SO and NSO youth relative to when they first entered the OYA facility. Further, depending on the length of incarceration, all youth living within a particular dorm have experienced the same environmental stimuli, which may create additional limits on the variety of juveniles' sexual fantasies. Given that the average length of stay in an OYA youth correctional facility is about 13 months (Oregon Youth Authority, 2020), exposure to the same environment may shape youth's fantasies in a very similar manner.

Alternatively, the absence of group differences in this study may accurately reflect a lack of significant differences between juvenile SOs' and NSOs' sexual fantasies. At present, in our country, there is easy access to pornography, the internet, and communication with peers, making potential stimuli for developing sexual fantasies 
much more readily available (Cooper et al., 2016). Today, across our country, it takes minimal effort to seek out stimuli that may prompt deviant sexual fantasies. With these stimuli so readily available to the juvenile population at large, adolescents may be exposed to many more similar, as opposed to different, sexual stimuli over time, resulting in juvenile offender groups (i.e., SO and NSO) experiencing largely similar sexual fantasies. Additionally, such easy access to sexual content through the internet and smartphones begs the question of the role of sexual fantasy for adolescents. Sexual fantasy requires intentional effort to maintain the fantasy; whereas now, adolescents could easily do an internet search and watch or view content related to their thought. If this is the case, clinicians may be overestimating the importance of deviant sexual fantasies revealed by SOs as an indicator of the need for "sex offender specific treatment (Howitt, 2004).”

Overall, these findings do support the identification of "deviant" sexual behavior in these offender groups, specifically solitary sexual behavior (i.e., SO) and voyeurism (i.e., NSO), as predictors of offender group membership, but they do not support deviant sexual fantasies as a predictor of offender group membership. Limitations in the data that was collected and the means by which analyses were conducted (i.e., covariate included and assigning group membership) indicate that future research should focus on exploring these two constructs in more depth.

Future directions. Several future directions merit consideration given this study's process and findings. First, future research should explore updating the measures used in this study. This investigation used the same version of the Sexual History Form- 
Revised (SHF-R; Kaufman, 2014) and Sexual Fantasy Questionnaire (SFQ; Kaufman, 1998) that was used in published research and last evaluated, psychometrically, in the late 1990s. Following the assumption that what is considered deviant behavior has changed over time, likely significantly, consideration should be given to updating measure items and reevaluating these measures with participants from the current generation (Larsen, 2013). Considering the low base rates of item endorsement for many participants on these measures, these questionnaires may be altered significantly by a psychometric reevaluation with a current juvenile sample. Additionally, it may be that that sexual behaviors and sexual fantasies that are relevant to youth today are actually missing from the current versions as well as some items no longer being pertinent (e.g., pornographic magazines [e.g., Hustler, Playboy]).

This study's measure of sexual fantasies produces a global indicator of fantasy. This scale includes sexual fantasies with varying degrees and types of "deviance." In contrast, the sexual history measure is broken down into a number of subscales that are much shorter and more specific. It is possible that offender subgroup differences may be related to more specific types of deviant fantasies and were masked by the use of a "global" scale. As such, future research should consider a more fine-grained analysis involving an exploration of the moderating effect of offender group on the relationship between more specific, deviant sexual fantasies (e.g., violent fantasies) and deviant sexual behaviors.

Offender group categorization was determined through identification by OYA or self-report on items measuring frequency of nonconsensual sexual behavior. Significant 
results may have been masked by incorrect identification of participants into offender groups. Future research may benefit from a more formal process for determining offender group placement, as opposed to most recent commitment offense. Verification of offender groups using court records or OYA files would provide the ability to create more "pure" offender groups. Additionally, the methods used to assign offenders to the two groups (i.e., SO and NSO) did not allow for the identification of "mixed" offenders. It's possible that offenders who have committed both sexual and nonsexual offenses may have a distinctive pattern with regard to either or both their sexual fantasies and their sexual behaviors.

While many of the analyses in this study did not reveal significant findings related to sexual fantasy, while examining the entire sample, it is possible that future investigations may reveal differences specific to particular offender sub-groups. The offender groups used in this study were broad-based (i.e., SO or NSO) and included participants who exhibited the full range of behaviors with regard to violence, coercion, crime frequency, etc. Important findings related to juvenile offender subgroups may have been masked by virtue of this study's focus on larger group differences. For example, in future studies, differences may emerge for offender subgroups who engaged in more violent sexual offenses or contact offense. Alternatively, differences may be revealed when comparing juvenile offender subgroups created based on their victims' demographic characteristics, such as, age of the victim, the victim's parental status (e.g., single parent, two parent), victim gender, and victim relationship to the offender. 
Future research should explore this study's finding that solitary sexual behavior predicts offender group membership, after controlling for nondeviant sexual behavior, in greater depth. The two items in this particular subscale that differentiated the groups were sexual acts with animals and masturbating alone. Research specific to masturbation would benefit from creating a better understanding of the context within which masturbation occurs. For example, does masturbation occur more frequently to deviant or nondeviant sexual fantasies? And how often is this solitary masturbation linked to future illegal or inappropriate behaviors that were the subject of the fantasy. It would also be helpful to further investigate the nature of SOs' reported sexual acts with animals. For example, it is plausible that these acts occur more frequently by youth living in rural settings, where access to farm animals is more likely and social interaction with same aged peers may be challenged by distance. Other factors that would be interesting for future research to explore are the role of self-regulation, sex as a coping strategy, and offenders' understanding of appropriate sexual behavior for their age as predictors of offender group membership (i.e., SO or NSO).

Finally, future studies should collect and contrast sexual fantasy and sexual behavior data from offenders who are supervised in the community, as well, as youth who are incarcerated, as these samples typically represent distinct subgroups of offenders (DiGorgio-Miller, 2007). To truly understand how juvenile offenders' sexual fantasies and sexual behavior may influence their criminal behavior, it is also important to obtain and compare their findings to those of age-appropriate community controls.

\section{Consensual and Nonconsensual Sexting}


This section will provide a discussion of the findings and future directions addressing the second goal of this study; exploring differences in sexting behaviors between juvenile SOs and NSOs. Significant differences were found between the two groups (i.e., SOs and NSOs) on consensual sexting behavior (i.e., Hypothesis 5a). Further examination indicated that this difference was primarily driven by one item. Juvenile NSOs endorsed 'Receiving a picture of someone's genitals/buttocks/breasts through text message/email/social media' more often than juvenile SOs.

The effect size for this difference is .033 , indicating that this difference may be trivial, even though it is statistically significant. That said, this finding is supported in the research literature. With significant advances in technology, sexting has become a normalized behavior, even in teens (Cooper et al., 2016). Adolescents report using sexting to flirt or gain attention from someone they are interested in engaging, as a way to maintain intimacy during periods of physical separation from their partner, and as a means of expressing mutual affection and arousal in anticipation of physical intimacy (Cooper et al. 2016). However, the social deficit model of sexual offending behavior suggests that SOs have difficultly achieving intimacy or developing relationships with same aged peers (Daleidan et al., 1998; Marshall et al., 1993). A greater frequency of consensual sexting involving photos by NSOs, may be evidence of this difficulty in SOs and may frame NSOs as "more normative" in this type of adolescent sexual behavior. Additionally, the data used for this study exhibited a non-linear relationship, which violates an assumption of the general linear model. This violation resulted in a loss of power to detect significant differences. Therefore, although only one item revealed 
significant differences, additional differences may exist on other items, that could not be detected in this study's analyses.

It is also important to consider that incarceration has likely limited (i.e., made it impossible) both groups of offenders' ability to engage in sexting. Access to phones and other electronic media is strictly prohibited inside of the correctional facility, without supervision. Moreover, depending upon their age, some youth may have been incarcerated at just the time when their social and interpersonal skills matured to the point where they would have been developing intimate relationships and had much more opportunity for sexting.

Cooper and colleagues (2016) have criticized the field for the lack of research on nonconsensual sexting behavior. Nonconsensual sexual behavior may face the same measurement and detection challenges as deviant behaviors. The low base rates of these behaviors reported by participants in this study may be indicative of nonconsensual sexting being a non-normative (i.e., deviant) behavior for adolescents. Alternatively, there may be a lack of understanding about what represents consent in these situations, muddying the distinction between consensual and nonconsensual sexting for study participants. First, Cooper and colleagues (2016) revealed that adolescents experience a great deal of peer pressure related to sexting. Additionally, it is possible that participants may view sexual images, in their possession, as theirs to distribute. It is possible that, if the image is of themselves, they do not see sending this image to an unwilling receiver as a violation of consent. If the image they are sending is of another person, they may be unlikely to see sexting as a violation of consent. 
Future directions. Research focused on sexting behaviors of adolescents is still emerging (Cooper et al., 2016). Therefore, further research to better understand subgroup differences and whether sexting has a role in offending behavior is needed. The items that were used to measure sexting were written for this study and would benefit from refinement. Multicollinearity indicated that several items were measuring the same dimensions. Additionally, these items were fairly vague and, if made more specific, they may be able to better differentiate the offender subgroups. Finally, it would be interesting to explore how the concept of consent manifests in sexting.

\section{Limitations}

This study has several important limitations, that should be considered. This section will describe each of these limitations, their potential impact to the study, and any strategies used to mitigate that impact. First, the data used for this study represents a cross-sectional design using retrospective, self-report data. The cross-sectional design does not allow causal conclusions to be drawn. For example, Hypothesis 3 explored the moderating effect of offender group on the relationship between sexual fantasy and sexual behavior. For this analysis, sexual fantasy was used as the independent variable and sexual behavior was used as the dependent variable. It is impossible to conclude, based on this data, if sexual fantasy temporally predicts sexual behavior, or vice versa. Additionally, due to the retrospective nature of the questions, it is assumed that participants could reliably recall the details of their historical fantasies and behavior. The items used for this study asked participants to recall their fantasies and behavior across their lifetime. Poor recall may have impacted participants responses. 
Second, it is possible that participants may have experienced participant fatigue, due to their involvement in a relatively long, multi-component study. In total, participants completed three survey packets with a total of 573 items. Surveys were completed in a single session that took approximately 1 hour and 30 minutes to complete. It is possible that some portion of the participants became bored or tired of filling out the questionnaire. A lack of sustained attention could have also impacted some participants' responses to the measures, particularly if they were not taking the time to thoughtfully respond to the items. To mitigate the potential for participant fatigue, participants were instructed to take as much time as they needed, were able to take breaks, and were given a snack halfway through the data collection.

The data used in this study was collected from juveniles, in Oregon, who had been adjudicated in the juvenile criminal justice system or convicted in the adult criminal justice system and incarcerated for their crimes. Therefore, these results can only be generalized to incarcerated juveniles in Oregon. Youth who are placed in youth correctional facilities tend to represent a greater risk to recidivate than youth who are placed in residential treatment facilities or who remain at home (DiGiorgio-Miller, 2007). It is possible that youth who were not caught, or those that were adjudicated or convicted and supervised in the community, have different patterns related to sexual fantasy and sexual behavior. As already noted, incarceration may also affect reports of sexual fantasies and sexual behavior because these youth have been removed from the community. This removal from the community significantly limits the potential stimuli that could inform the development of sexual fantasies. In addition, incarceration clearly 
limits the potential sexual behavior over the duration of their stay in the youth correctional facility.

The logistical arrangement of the data collection process, combined with the sensitive nature of the survey, warrants discussion, as well. Limitations of physical space and a need to avoid disruption of day-to-day facility operations, resulted in a less than ideal data collection environment. Data was collected from participants on their living units. Each living unit houses up to 25 young men, on any given day. While the unit and research staff did their best to maximize the use of the space, limited space required participants to sit near each other at shared tables while they completed their packets. The risk of peers seeing their responses at shared tables and knowing about their sexual fantasies or experiences may have impacted their responses. Moreover, the topic of sex may be embarrassing considering the developmental stage of study participants. There may be feelings of inadequacy if they lack sexual experience, or feelings of shame if they have interests or behaviors that are not socially acceptable. On the other hand, some participants may be prone to embellish their sexual experience to enhance their status. In an attempt to control for biased responding, participants completed a shortened version of the Marlowe-Crown Social Desirability Scale (Reynold, 1982).

Further, the SHF-R (Kaufman, 2014) and the SFQ (Kaufman, 1998) ask participants to report on very sensitive information about their sexual behaviors and fantasies, some of which, may not be known to OYA. Participants may have withheld certain information due to a concern that will endure additional consequence if they provide information that is not known to OYA or does not align with their treatment 
progress (Abel et al., 1987). To mitigate these concerns, participants were told, before data collection, that their responses were anonymous. They were asked not to provide their name or any other identifiable information on any of their surveys, or the envelope that they submitted their final surveys in, and participants were told that OYA unit staff would not have access to any of their survey information. Finally, participants were given as much space as the setting allowed to afford them privacy in completing the measures.

As discussed previously, offender group categorization was determined by OYA based on crime type and treatment need. However, the possibility exists that participant groups were not "pure" in the sense that all juveniles who had committed sexual offenses were identified as SOs. The primary point of concern lies in the fact that juveniles who had committed, but were not caught for and did not disclose sexual offenses, were not identified as SOs for this study. With underreporting being an issue for all forms of sexual violence, it becomes difficult to have confidence that all NSOs in a participant sample have not engaged in sexual offending. This may confound findings, making significant differences more difficult to find.

\section{Study Implications}

This study’s findings offer several interesting implications. This section will present implication for theory related to juvenile sexual offending and juvenile SO treatment, prevention efforts, and policy. Acknowledging statistical limitations, the lack of group differences noted in this study has implications for theory. Currently, the field is collecting research to determine if juvenile SOs are "generalist" (i.e., there are few basic differences between youth who commit different types of crimes) or "specialist" (i.e., 
youth require different treatment based on the type of crimes they commit) offenders. According to Seto and Lalumiere (2010), one of the key areas lacking evidence to inform this question of juvenile offender differentiation is the role of sexuality in offending. Study findings lend themselves to support the "generalist" perspective and would suggest that sexual offending is a manifestation of general antisocial tendencies and may not have specific sexuality-related etiological risk factors (Pullman \& Seto, 2012; Seto \& Lalumiere, 2010). While multiple replications would be required to make a strong case for a true lack of group differences (i.e., SO vs. NSO), such a finding in support of juvenile SOs as "generalist" offenders would make a strong case for the re-evaluation of all current SO specific treatment requirements, as well as bolstering the case for more skills-based and developmentally appropriate treatment targets.

As previously noted, treatment has focused on deviant sexual fantasies and behavior as etiological factors specific to sexual offending and as primary treatment targets for those who have offended sexually. Significant findings, related to deviant sexual behavior predicting offender group membership, and nonsignificant findings, related to sexual fantasy, have interesting implications for thinking about treatment. Historically, treatment efforts have targeted the content and number of deviant sexual fantasies SOs report. However, as opposed to targeting all deviant sexual fantasies, treatment providers should focus, specifically, on the sexual fantasies to which juveniles are masturbating. According to the Conditioning Theory of Deviant Sexual Preference and Behavior, as fantasies have repeated association with orgasm, the desire to act them out in real life becomes greater (Laws \& Marshall, 1990; Marshall \& Eccles, 1993; 
Marshall \& Marshall, 2000). As such, consideration should be given to attaching more importance to the treatment implications of juveniles who are masturbating to violent, non-consensual, or otherwise "deviant" sexual fantasies.

Overall, the results of this study, related to sexual fantasy, were not significant. That is to say that differences between SOs and NSOs on sexual fantasies were not found for this sample of juveniles. While study sampling, measurement, and/or methodology may be responsible for the lack of group differences, it is also possible that study findings reflect a true lack of differences on this important dimension. If these findings can be replicated, they challenge the long-standing and widely accepted idea that juvenile sex offending is rooted in deviant sexual fantasies and/or an aberrant sexual behavior. If replications of this study confirm this not to be the case, it would have far reaching implications for reconsideration of core juvenile offender treatment strategies. Evidence that juveniles' deviant sexual fantasies are not at the heart of their sex offending would give greater credence reducing treatment attention in this regard and devoting precious staff time to addressing prosocial skills-building strategies, which have stronger empirical support and the potential to positively impact a broad range of juveniles' future opportunities (e.g., housing, socialization, employment, community reintegration, creating positive intimate relationships; Ralph 2016).

Over the past several years, OYA has been implementing a Positive Human Development approach (Oregon Youth Authority, 2019). This strategy treats youth in their care as developing individuals in need of guidance and skills enhancement, as opposed to criminals to be fixed. Treatment targets focus on skill building for all youth, 
with an emphasis on healthy relationships, appropriate interpersonal boundaries, coping strategies, and positive self-regulation. Identifying and implementing treatment curricula that continue to reinforce these concepts, while minimizing efforts to address deviant sexual behaviors and fantasies would provide youth with the tools necessary to be successful, rather than shaming them for sexuality that may, in fact, be normative for adolescents (Worling, 2012).

Confirming the results of this study may also allow for greater emphasis on factors that contribute to juvenile sex offending that are more immediately amenable to the development of effective prevention approaches. For example, prevention dollars may be better spent on teaching children and teens healthy sexuality, healthy boundaries, selfregulation and addressing situational factors that create unhealthy conditions making it easier for sexual abuse to occur (e.g., a lack of adequate staff/volunteer training in youth serving organizations; Kaufman et al., 2019; Schneider \& Hirsch, 2018).

Finally, such findings would have important implications for modified policy directions, as well. The majority of policies that guide juvenile SOs' registration as sex offenders, housing restrictions, and restrictive placements for treatment are rooted in justice system models based on the premise that the etiology of their offending is based on their deviant sexuality, which also fuels left-over public fear from the "superpredator" era of the late 1980s (Letourneau \& Caldwell, 2013). The result of these fears has been the enactment of punitive policies with long lasting and severe effects on juveniles who have sexually offended (Letourneau \& Caldwell, 2013). If these findings can be replicated (i.e., a lack of group differences), then education and advocacy may 
lead to a reform of current policies in favor of a return to more productive restorative justice strategies that foster the rehabilitation of juvenile SOs with an eye toward their successful long-term reintegration into society.

\section{Summary}

This study attempted to address a current gap in the literature related to how juvenile SOs and NSOs differ in their sexual fantasies and sexual behavior. The limited research investigating these differences is outdated, with the most recent studies having been conducted in the late 1990s (i.e., Daleidan et al., 1998; Fagan \& Wexler, 1988). In the intervening time, there have been significant advances in technology, as well as increased access to pornography, the internet, and communication through text messaging, emails, and social media. Having a better understanding of how deviance has changed over time and how these two groups of offenders (i.e., SO and NSO) differ is important to identifying appropriate treatment targets for juvenile SOs and for better informing prevention efforts. Significant findings related to deviant sexual behavior, help treatment professionals to narrow their focus from an emphasis on all deviant sexual fantasies, to those that are being repeatedly masturbated to over time. While this study has a number of limitations that should be considered when interpreting its results, if future replications confirm a lack of differences between SO and NSO groups on sexual fantasy, this will mark an important shift in our understanding of key etiological factors related to juvenile sex offending. In turn, this will significantly impact directions for treatment planning as well as the prevention of sexual offending perpetrated by juveniles. 
Table 1. Sexual Fantasy Subscales

Global Non-Deviance (49 items)

Hugging someone

Comforting someone

Making out

Being excited by another person's body

Loving someone

Getting a partner really sexually excited

Someone you find sexually attractive trying to get you sexually aroused

Getting married

Trying to arouse someone so they will want to have sex with you

Sex that lasts for hours

Gaining the love of a rejecting lover

Reliving past sexual experiences

Licking

Petting

Sucking

Receiving oral sex

Giving oral sex

Taking someone's clothes off

Someone taking your clothes off

Kissing a partner's mouth

Holding a partner's hand

Kissing your partner's penis, vagina, buttock, or breasts

Masturbating your partner

Having a partner masturbating you

Touching a partner's genital area

Touching other non-genital parts of a partner's body

Partner touching your genital

Partner touching other non-genital parts of your body

Intercourse in unusual positions

Watching a partner undress

Having a partner take charge sexually

Sex with two or more people

Sex with teenagers (12-17 years-old)

Sex with people 18 years and older

Sexual activity with friends

Sex with a oved partner

Sex with someone you know, but have not had sex with

Sex with a virgin

Sex with someone of a different race

Sex with a former partner

Sex with a famous person

Having multiple orgasms

Asking for what you want during sex

Having sex somewhere in the house other than the bedroom

Having sex in a secluded place

Sex in a romantic setting (e.g., on the beach, honeymoon)

Sex in an exotic setting (e.g., wilderness, foreign country)

Sex in unusual locations (e.g., rooftop, library)

Global Deviance (49 items)

Dressing in costumes during sexual activity 
Someone using objects to get you excited

Using objects to get someone else excited

Receiving anal sex

Spanking someone

Being spanked by someone

Controlling or intimidating a partner

Yelling at a partner

Ignoring a partner's protests

Pinching during sexual activity

Using weapons during sexual activity

Hitting someone during sexual activity

Being forced to do something sexual

Forcing someone to do something sexual

Hurting partner during sexual activity

Being hurt by someone during sexual activity

Being tied up

Tying someone up

Threatening to hurt a partner

Partner threatening to hurt you during sexual activity

Raping someone

Being raped

Being sexually degraded (called names, laughed at, etc.)

Sexually degrading a partner (calling them names, laughing at them, etc.)

Being tortured by a sex partner

Being whipped or beaten by a partner

Whipping or beating a partner

Torturing a sex partner

Exposing yourself

Setting fires

Scaring someone

Making prank/obscene phone calls

Sex with a dead person

Paying money for sex

Being paid money for sex

Watching others have sex

Having sex with a relative

Kidnapping someone for sexual purposes

Being kidnapped by someone for sexual purposes

Urination (peeing) as part of sexual activity

Sex with young children (under 6 years old)

Sex with children (6-11 years old)

Sexual activity with animals

Sex with someone who has a handicap

Being sexually uninhibited

Fantasizing that you are of the opposite sex

Dressing in clothes of the opposite sex

Having sex where there is a danger of being caught 
Table 2. Sexual History Subscales ${ }^{4}$

\section{Typical/Consensual (11 items)}

Lying on a partner while you are clothed

Stroking and petting a partner's genitals (penis or vagina)

Lying on a partner while you are naked

Vaginal intercourse (penis placed in vagina)

Having a partner rub or stroke your genitals

Orally stimulating a partner's genitals (kissing or licking)

Kissing sensitive, non-genital body areas

Having sensitive, non-genital areas stroked or rubbed

Stroking or rubbing a partner's sensitive non-genital areas

Having your genitals orally stimulated (kissed or licked)

Having heterosexual (male-female) sex

\section{Atypical/Consensual (4 items)}

Anal intercourse (penis placed inside anus)

Putting your finger inside a partner's anus

Paying a partner to perform sexual acts with you

Having homosexual sex (male-male or female-female)

\section{Aggressive/Consensual (3 items)}

Physically hurting, humiliating, or embarrassing a partner

A partner hurting you physically, humiliating or embarrassing you

Threatening or frightening a partner

\section{Typical/Non-Consensual (12 items)}

Lying on a partner while you are clothed

Stroking and petting a partner's genitals (penis or vagina)

Lying on a partner while you are naked

Vaginal intercourse (penis placed in vagina)

Having a partner rub or stroke your genitals

Orally stimulating a partner's genitals (kissing or licking)

Kissing sensitive, non-genital body areas

Having sensitive, non-genital areas stroked or rubbed

Stroking or rubbing a partner's sensitive non-genital areas

Having your genitals orally stimulated (kissed or licked)

Putting your finger inside a partner's vagina

Hugging while undressed

\section{Voyeurism (4 items)}

Taking pictures or movies/videos of someone nude

Taking pictures or movies/videos of people engaging in sexual activity

Secretly watching others have intercourse

Flashing or exposing your genitals (penis or vagina)

\section{Paraphilias (15 items)}

A partner paying you to perform sexual acts

Physically hurting, humiliating, or embarrassing a partner

\footnotetext{
${ }^{4}$ Titles and items in each subscale are preserved from the Factor Analysis published by Daleidan et al., 1998. Social norms change over time and it is recognized that the titles of the subscales (e.g., "Atypical") may be outdated. It is also recognized that subscales were determined based on factor loadings.
} 
Threatening or frightening a partner

Calling a stranger on the phone to talk dirty

Rubbing against or touching a stranger in a sexual way

Touching a stranger in a way against their will

Forcing intercourse

Forcing other sexual acts

Masturbating in a public place

Dressing like someone of the opposite sex

Becoming sexually excited by watching a fire

Becoming sexually excited by stealing something

Watching someone dress or undress without them knowing

Flashing or exposing your genitals (penis or vagina)

Flashing or exposing your buttocks

Solitary Sex Acts (4 items)

Sexual contact with an animal

Masturbating alone

Looking at erotic magazines (e.g., Playboy, Hustler)

Looking at pictures or movies/videos of someone naked

\section{Sexting (7 items)}

Sending a picture of your penis or buttocks to someone through text message/email/online chat (e.g., Facebook)

Receiving a picture of someone's genitals/buttocks/breasts through text message/email/online chat (e.g., Facebook)

Sending a naked picture of someone other than yourself through text message/email/online chat (e.g., Facebook)

Sending a sexual video of yourself through text message/email/online chat (e.g., Facebook)

Sending a sexual video of someone else through text message/email/online chat (e.g., Facebook)

Sending a sexual video of you and another person through text message/email/online chat (e.g., Facebook)

Sending messages with sexual content through text message/email/online chat (e.g., Facebook) 
Table 3. Correlation Matrix - Determining Covariates

\begin{tabular}{lcc}
\hline & $\begin{array}{c}\text { History of Sexual Abuse } \\
\text { Victimization }\end{array}$ & Social Desirability \\
\hline Offender group membership & -.016 & -.058 \\
Typical Consensual & .015 & .069 \\
Atypical Consensual & -.085 & -.008 \\
Aggressive Consensual & -.037 & .012 \\
Typical Nonconsensual & -.044 & $-.154^{*}$ \\
Voyeurism & -.034 & -.112 \\
Paraphilias & -.007 & $-.202^{* *}$ \\
Solitary Sex & .045 & $-.219^{* *}$ \\
Sexting Consensual & .013 & -.088 \\
Sexting Nonconsensual & -.031 & -.144 \\
Non-Deviant 12 Months & .040 & -.087 \\
Non-Deviant Lifetime & .062 & -.034 \\
Deviant 12 Months & .095 & $-.146^{*}$ \\
Deviant Lifetime & .095 & $-.160^{*}$ \\
\hline
\end{tabular}

$* p<.05$

$* * p<.01$ 
Table 4. Subscale Alpha Coefficients

\begin{tabular}{lcc}
\hline Subscale & Reliability & Number of Items \\
\hline Typical Consensual & .94 & 11 \\
Atypical Consensual & .65 & 4 \\
Aggressive Consensual & .88 & 3 \\
Typical Non-Consensual & .96 & 12 \\
Yoyeurism & .65 & 4 \\
Paraphilias & .81 & 15 \\
Solitary Sex Acts & .72 & 4 \\
Sexting Consensual & .88 & 7 \\
Sexting Non-Consensual & .84 & 7 \\
Global Non-Deviance 12 Months & .98 & 48 \\
Global Non-Deviance Lifetime & .97 & 48 \\
Global Deviance 12 Months & .96 & 48 \\
Global Deviance Lifetime & .96 & 48 \\
Marlowe Crown Social Desirability Scale - Short Form Revised & .70 & 10 \\
\hline
\end{tabular}


Table 5. Subscale Means and Standard Deviations

\begin{tabular}{|c|c|c|}
\hline \multirow[b]{2}{*}{ Item } & \multicolumn{2}{|c|}{ Means (SD) } \\
\hline & JNSO & JSO \\
\hline \multicolumn{3}{|l|}{ Sexual Fantasy } \\
\hline Nondeviant 12-Month Sexual Fantasies* & $.07(.04)$ & $.06(.03)$ \\
\hline Deviant 12-Month Sexual Fantasies & $.01(.02)$ & $.01(.02)$ \\
\hline Nondeviant Lifetime Sexual Fantasies & $.06(.03)$ & $.06(.03)$ \\
\hline Deviant Lifetime Sexual Fantasies & $.01(.02)$ & $.01(.01)$ \\
\hline \multicolumn{3}{|l|}{ Sexual History } \\
\hline Typical Consensual* & $.33(.15)$ & $.26(.16)$ \\
\hline Atypical Consensual & $.23(.29)$ & $.23(.27)$ \\
\hline Aggressive Consensual & $.08(.31)$ & $.10(.31)$ \\
\hline Voyeurism* & $.20(.25)$ & $.12(.21)$ \\
\hline Paraphilias & $.01(.03)$ & $.02(.04)$ \\
\hline Solitary Sex* & $.64(.38)$ & $.76(.30)$ \\
\hline Typical Nonconsensual* & $.01(.05)$ & $.05(.08)$ \\
\hline
\end{tabular}

$* p<.05$ 
Table 6. Hypothesis 2a: Comparison of Log-Likelihood Ratios

\begin{tabular}{|c|c|c|c|c|c|c|c|}
\hline & \multicolumn{3}{|c|}{ Goodness of Fit } & \multicolumn{4}{|c|}{ Log-Likelihood Ratios } \\
\hline & $\chi^{2}$ & $d f$ & $p$ & $\chi^{2}$ & $d f$ & $p$ & $\begin{array}{c}\text { Nagelkerke } \\
R^{2}\end{array}$ \\
\hline $\begin{array}{l}\text { Step 1: Covariate - Social } \\
\text { Desirability }\end{array}$ & 17.60 & 7 & .014 & .72 & 1 & 40 & .00 \\
\hline $\begin{array}{l}\text { Step 2: Nondeviant Lifetime } \\
\text { Fantasies }\end{array}$ & 5.69 & 8 & .68 & 2.97 & 1 & .09 & .02 \\
\hline $\begin{array}{l}\text { Step 3: Deviant Lifetime } \\
\text { Fantasies }\end{array}$ & 5.40 & 8 & .71 & .17 & 1 & .68 & .02 \\
\hline
\end{tabular}


Table 7. Hypothesis 2b: Comparison of Log-Likelihood Ratios

\begin{tabular}{|c|c|c|c|c|c|c|c|}
\hline & \multicolumn{3}{|c|}{ Goodness of Fit } & \multicolumn{4}{|c|}{ Log-Likelihood Ratios } \\
\hline & $\chi^{2}$ & $d f$ & $p$ & $\chi^{2}$ & $d f$ & $p$ & $\begin{array}{c}\text { Nagelkerke } \\
R^{2}\end{array}$ \\
\hline $\begin{array}{l}\text { Step 1: Covariate - Social } \\
\text { Desirability }\end{array}$ & 18.91 & 7 & .02 & .66 & 1 & .42 & .00 \\
\hline $\begin{array}{l}\text { Step 2: Nondeviant 12-Month } \\
\text { Fantasies }\end{array}$ & 20.06 & 8 & .01 & 5.91 & 1 & .02 & .04 \\
\hline $\begin{array}{l}\text { Step 3: Deviant 12-Month } \\
\text { Fantasies }\end{array}$ & 9.81 & 8 & .28 & .334 & 1 & .56 & .04 \\
\hline
\end{tabular}


Table 8. Hypothesis 3: Comparison of Log-Likelihood Ratios

\begin{tabular}{|c|c|c|c|c|c|c|c|}
\hline & \multicolumn{3}{|c|}{ Goodness of Fit } & \multicolumn{4}{|c|}{ Log-Likelihood Ratios } \\
\hline & $\chi^{2}$ & $d f$ & $p$ & $\chi^{2}$ & $d f$ & $p$ & Nagelkerke $R^{2}$ \\
\hline $\begin{array}{l}\text { Step 1: Covariate - Social } \\
\text { Desirability }\end{array}$ & 18.69 & 7 & .01 & .58 & 1 & .45 & .00 \\
\hline $\begin{array}{l}\text { Step 2: Nondeviant Sexual } \\
\text { Behavior }\end{array}$ & 7.24 & 8 & .51 & 8.74 & 1 & .00 & .05 \\
\hline $\begin{array}{l}\text { Step 3: Deviant Sexual } \\
\text { Behavior }\end{array}$ & 7.76 & 8 & .46 & 30.49 & 5 & .00 & .22 \\
\hline
\end{tabular}


Table 9. Logistic Regression Predicting Group Membership Based on Social Desirability, Nondeviant Sexual Behavior, and Deviant Sexual Behavior.

\begin{tabular}{lccccccccc}
\hline & \multicolumn{1}{c}{} & \multicolumn{1}{c}{} & & & & & \multicolumn{2}{c}{$95 \%$ CI for Odds } \\
\cline { 8 - 10 } & $B$ & SE & Wald & $d f$ & $p$ & Odds Ratio & Lower & Upper \\
\hline Social Desirability & -.02 & .06 & .06 & 1 & .81 & .99 & .87 & 1.11 \\
Typical Consensual & -3.25 & 1.19 & 7.43 & 1 & .01 & .04 & .00 & .40 \\
Atypical Consensual & 1.15 & .77 & 2.22 & 1 & .14 & 3.16 & .69 & 14.40 \\
Aggressive Consensual & .14 & .77 & .04 & 1 & .85 & 1.16 & .26 & 5.24 \\
Paraphilias & 11.01 & 7.52 & 2.15 & 1 & .14 & 60475.22 & .02 & $1.51 \mathrm{E}+11$ \\
Solitary Sex & 1.91 & .51 & 13.81 & 1 & .00 & 6.74 & 2.46 & 18.43 \\
Voyeurism & -4.16 & 1.12 & 13.72 & 1 & .00 & .02 & .00 & .14 \\
Constant & -.77 & .53 & 2.11 & 1 & .15 & .46 & & \\
\hline
\end{tabular}


Table 10. Correlation Matrix - Consensual Sexting Items

\begin{tabular}{lccccccc}
\hline \multicolumn{1}{l}{} & 1 & 2 & 3 & 4 & 5 & 6 & 7 \\
\hline $\begin{array}{l}\text { 1. Sending a picture of your penis or } \\
\text { buttocks to someone }\end{array}$ & 1 & & & & & & \\
$\begin{array}{l}\text { 2. Receiving a picture of someone's } \\
\text { genitals/buttocks/breasts }\end{array}$ & $.69^{* *}$ & 1 & & & & \\
$\begin{array}{l}\text { 3. Sending a naked picture of } \\
\text { someone other than yourself }\end{array}$ & $.42^{* *}$ & $.42^{* *}$ & 1 & & & \\
$\begin{array}{l}\text { 4. Sending a sexual video of your } \\
\text { penis or buttocks }\end{array}$ & $.65^{* *}$ & $.45^{* *}$ & $.48^{* *}$ & 1 & & \\
$\begin{array}{l}\text { 5. Sending a sexual video of } \\
\text { someone's genitals/buttocks/breasts }\end{array}$ & $.40^{* *}$ & $.38^{* *}$ & $.77^{* *}$ & $.55^{* *}$ & 1 & \\
$\begin{array}{l}\text { 6. Sending a sexual video of you and } \\
\text { another person }\end{array}$ & $.51^{* *}$ & $.40^{* *}$ & $.63^{* *}$ & $.66^{* *}$ & $.73^{* *}$ & 1 & \\
$\begin{array}{l}\text { 7. Sending messages with sexual } \\
\text { content }\end{array}$ & $.68^{* *}$ & $.71^{* *}$ & $.44^{* *}$ & $.49^{* *}$ & $.40^{* *}$ & $.42^{* *}$ & 1 \\
\hline & & & & & & & \\
\hline
\end{tabular}


Table 11. Means and Standard Deviations - Consensual Sexting Items

\begin{tabular}{lcc}
\hline & \multicolumn{2}{c}{ Means (SD) } \\
\cline { 2 - 3 } Item & JNSO & JSO \\
\hline Sending a picture of your penis or buttocks to someone & $2.298(2.010)$ & $2.081(1.966)$ \\
Receiving a picture of someone's genitals/buttocks/breasts* & $3.764(2.061)$ & $2.942(2.155)$ \\
Sending a naked picture of someone other than yourself & $.933(1.669)$ & $.721(1.460)$ \\
Sending a sexual video of your penis or buttocks & $1.197(1.835)$ & $.872(1.686)$ \\
Sending a sexual video of you and another person & $.893(1.689)$ & $.488(1.344)$ \\
\hline
\end{tabular}

$* p<.05$ 
Table 12. Item Frequencies - Nonconsensual Sexting Items

\begin{tabular}{|c|c|c|c|c|c|c|c|}
\hline Item & $\begin{array}{c}0 \\
\text { Never in } \\
\text { my life }\end{array}$ & $\begin{array}{l}1 \\
\text { One or two } \\
\text { times }\end{array}$ & $\begin{array}{c}2 \\
\text { About } 3 \\
\text { or } 4 \\
\text { times } \\
\end{array}$ & $\begin{array}{c}3 \\
\text { About } 5 \text { to } \\
10 \text { times }\end{array}$ & $\begin{array}{c}4 \\
\text { About } 11 \\
\text { to } 20\end{array}$ & $\begin{array}{l}5 \\
\text { More than } \\
50 \text { times }\end{array}$ & Missing \\
\hline $\begin{array}{l}\text { Sending a picture of your } \\
\text { penis or buttocks to } \\
\text { someone }\end{array}$ & 244 & 11 & 3 & 1 & 1 & 0 & 8 \\
\hline $\begin{array}{l}\text { Receiving a picture of } \\
\text { someone's } \\
\text { genitals/buttocks/breasts }\end{array}$ & 240 & 6 & 8 & 2 & 2 & 2 & 8 \\
\hline $\begin{array}{l}\text { Sending a naked picture of } \\
\text { someone other than } \\
\text { yourself }\end{array}$ & 248 & 6 & 3 & 0 & 1 & 1 & 9 \\
\hline $\begin{array}{l}\text { Sending a sexual video of } \\
\text { your penis or buttocks }\end{array}$ & 251 & 4 & 2 & 1 & 1 & 0 & 9 \\
\hline $\begin{array}{l}\text { Sending a sexual video of } \\
\text { someone's } \\
\text { genitals/buttocks/breasts }\end{array}$ & 256 & 1 & 1 & 1 & 0 & 0 & 9 \\
\hline $\begin{array}{l}\text { Sending a sexual video of } \\
\text { you and another person }\end{array}$ & 256 & 1 & 1 & 0 & 1 & 0 & 9 \\
\hline $\begin{array}{l}\text { Sending messages with } \\
\text { sexual content }\end{array}$ & 240 & 7 & 4 & 1 & 2 & 2 & 12 \\
\hline
\end{tabular}

$N=268$ 
Table 13. Correlation Matrix Nonconsensual Sexting Items

\begin{tabular}{|c|c|c|c|c|c|c|c|}
\hline & 1 & 2 & 3 & 4 & 5 & 6 & 7 \\
\hline $\begin{array}{l}\text { 1. Sending a picture of your penis } \\
\text { or buttocks to someone }\end{array}$ & 1 & & & & & & \\
\hline $\begin{array}{l}\text { 2. Receiving a picture of someone's } \\
\text { genitals/buttocks/breasts }\end{array}$ & $.72 * *$ & 1 & & & & & \\
\hline $\begin{array}{l}\text { 3. Sending a naked picture of } \\
\text { someone other than yourself }\end{array}$ & $.15^{*}$ & $.24 * *$ & 1 & & & & \\
\hline $\begin{array}{l}\text { 4. Sending a sexual video of your } \\
\text { penis or buttocks }\end{array}$ & $.54 * *$ & $.52 * *$ & .04 & 1 & & & \\
\hline $\begin{array}{l}\text { 5. Sending a sexual video of } \\
\text { someone's } \\
\text { genitals/buttocks/breasts }\end{array}$ & $.53^{* *}$ & $.50 * *$ & $.15^{*}$ & $.52 * *$ & 1 & & \\
\hline $\begin{array}{l}\text { 6. Sending a sexual video of you } \\
\text { and another person }\end{array}$ & $.79 * *$ & $.66 * *$ & .01 & $.61 * *$ & $.76^{* *}$ & 1 & \\
\hline $\begin{array}{l}\text { 7. Sending messages with sexual } \\
\text { content }\end{array}$ & $.62 * *$ & $.78^{* *}$ & $.14 *$ & $.70^{* *}$ & $.64 * *$ & $.71 * *$ & 1 \\
\hline
\end{tabular}


Figure 1. Moderation of the Relationship Between Deviant Lifetime Fantasies and Aggressive Consensual Sexual Behavior by Offender Group (i.e., SO vs. NSO)

2

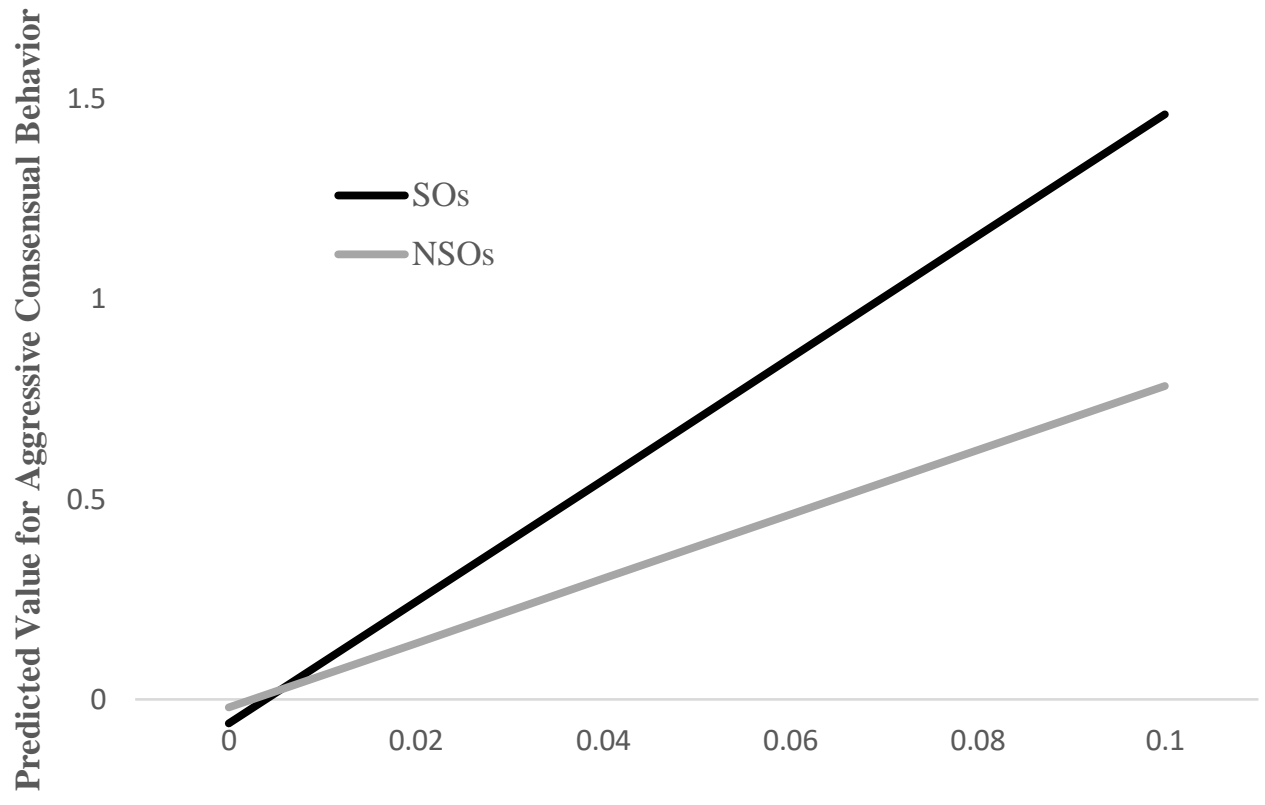

$-0.5$ 


\section{References}

Abel, G.G. \& Blanchard, E.B. (1974). The role of fantasy in the treatment of sexual deviation. Archives of General Psychiatry, 30, 467-475.

Anderson, M \& Jiang, J. (2018). Teens, social media \& technology 2018. Pew Research Center. Retrieved from https://www.pewresearch.org/internet/2018/05/31/teenssocial-media-technology-2018/

Anderson, M. (2019). Mobile technology and home broadband 2019. Pew Research Center. Retrieved from https://www.pewresearch.org/internet/2019/06/13/mobiletechnology-and-home-broadband-2019/

Arain, M., Haque, M., Johal, L., Mathur, P., Nel, W., Rais, A., Shandu, R., \& Sharma, S. (2013). Maturation of the adolescent brain. Neuropsychiatric Disease and Treatment, 9, 449-461.

Aylwin, A.S., Reddon, J.R., \& Burke, A.R. (2005). Sexual fantasies of adolescent male sex offenders in residential treatment: A descriptive study. Archives of Sexual Behavior, 34(2), 231-239

Bandura, A. (1973). Aggression: A social learning analysis. Englewood Cliffs, NJ: Prentice-Hall.

Bartels, R.M. \& Gannon, T.A. (2011). Understanding the sexual fantasies of sex offenders and their correlates. Aggression and Violent Behavior, 16, 551-561.

Beitchman, J.H., Zucker, K.J., Hood, J.E., DaCosta, G.A., Akman, D, \& Cassavia, E. (1992). A review of the long-term effects of child sexual abuse. Child Abuse \& Neglect, 16, 101-118. 
Browne, A. \& Finkelhor, D. (1986). Impact of child sexual abuse: a review of the research. Psychological Bulletin, 99(1), 66-77.

Buren, J. \& Lunde, C. (2018). Sexting among adolescents: A nuanced and gendered online challenge for young people. Computers in Human Behavior, 85, 210-217.

Cale, J., Lussier, P., McCuish, E., \& Corrado, R. (2015). The prevalence of psychopathic personality disturbance among incarcerated youth: Comparing serious, chronic, violent, and sex offenders. Journal of Criminal Justice, 34(4), 337-344.

Calleja, N. (2013). Integrating research into practice: The forward-focused model of adolescent sexual behavior treatment. Aggression and Violent Behavior, 18, 686694.

Cauffman, N. \& Steinberg, L. (2012). Emerging findings from research on adolescent development and juvenile justice. Victims \& Offenders, 7(4), 428-449.

Centers for Disease Control and Prevention. (2004). Sexual violence prevention: Beginning the dialogue. Atlanta, GA: Centers for Disease Control and Prevention. Centers for Disease Control and Prevention. (2010). The levels of prevention. Centers for Disease Control and Prevention. Retrieved from https://vetoviolence.cdc.gov/levels-prevention

Chaffin, M. (2008). Our minds are made up--Don't confuse us with the facts: Commentary on policies concerning children with sexual behavior problems and juvenile sex offenders. Child Maltreatment, 13, 110-121.

Cooper, A. (1998). Sexuality and the internet: Surfing into the new millennium. CyberPsychology \& Behavior, 1(2), 181-187. 
Cooper, A., Delmonico, D.L., \& Burg, R. (2000). Cybersex users, abusers, and compulsives: New findings and implications. Sexual Addiction \& Compulsivity, 7, $5-29$.

Cooper, K., Quayle, E., Jonsson, L., \& Svedin, C.G. (2016). Adolescents and self-taken sexual images: A review of the literature. Computers in Human Behavior, 55, 706-716.

Daleiden, E.L., Kaufman, K.L., Hilliker, D.R., \& O’Neil, J.N. (1998). The sexual histories and sexual fantasies of youthful males: A comparison of sexual offending, nonsexual offending, and nonoffending groups. Sexual Abuse: A Journal of Research and Treatment, 10, 195-209.

Darlington, R.B. \& Hayes, A.F. (2017). Regression analysis and linear models: Concepts, applications, and implementation. New York: The Guildford Press.

De Block, A. \& Adriaens, P.R. (2013). Pathologizing sexual deviance: A history. Journal of Sex Research, 50(3-4), 276-298.

de Graaf, T.A. \& Stack, A.T. (2011). Null results in TMA: From absence of evidence to evidence of absence. Neuroscience and Biobehavioral Reviews, 35, 871-877.

DiGiorgio-Miller, J. (2007). Emotional variables and deviant sexual fantasies in adolescent sex offenders. The Journal of Psychiatry and Law, 35, 109-124.

Earp, B.D. (2017). The need for reporting negative results - a 90 year update. Journal of Clinical and Translation Research, 3(S2), 344-347. 
Epstein, M. \& Ward, L.M. (2008). “Always use protection”: Communication boys receive about sex from parents, peers, and the media. $J$ Youth Adolescence, 37, 113-126

Fagan, J. \& Wexler, S. (1988). Explanations of sexual assault among violent delinquents. Journal of Adolescent Research, 3, 363-385.

Fang, X., Brown, D.S., Florence, C.S., \& Mercy, J.A. (2012). The economic burden of child maltreatment in the United States and implications for prevention. Child Abuse \& Neglect, 36(2), 156-165.

Finkelhor, D. (1994). Current Information on the Scope and Nature of Child Sexual Abuse. The Future of Children, 4(2), 31-53.

Finkelhor, D. \& Jones, L. (2006). Why have child maltreatment and child victimization declined? Journal of Social Issues, 62(4), 685-716.

Finkelhor, D., Ormrod, R., \& Chaffin, M. (2009). Juveniles who commit sex offenses against minors. U.S. Department of Justice, Office of Justice Programs, Office of Juvenile Justice and Delinquency Prevention. Washington, DC.

Gardner, M. \& Steinberg, L. (2005). Peer influence on risk taking, risk preference, and risky decision making in adolescence and adulthood: An experimental study. Developmental Psychology, 41(4), 625-635.

Gee, D.G., Devilly, G.J., \& Ward, T. (2004). The content of sexual fantasies for sexual offenders. Sexual Abuse: A Journal of Research and Treatment, 16, 315-331.

Gee, D.G., Ward, T., \& Eccleston, L. (2003). The function of sexual fantasies for sexual offenders: A preliminary model. Behaviour Change, 20, 44-60. 
Harms, C. \& Lakens, D. (2018). Making 'null effects' informative: statistical techniques and inferential frameworks. Journal of Clinical and Translational Research, 3(S2), 382-393.

Harris, D.A., Mazerolle, P., \& Knight, R.A. (2009). Understanding male sexual offending: A comparison of general and specialist theories. Criminal Justice and Behavior, 36, 1051-1069.

Hensley, C., Tallichet, S.E., \& Singer, S.D. (2006). Exploring the possible link between childhood and adolescent bestiality and interpersonal violence. Journal of Interpersonal Violence, 21(7), 910-923.

Howitt, D. (2004). What is the role of fantasy in sexual offending? Criminal Behaviour and Mental Health, 14, 182-188.

Human Rights Watch, (2013). Raised on the registry: The irreparable harm of placing children on sex offender registries in the US. Washington, D.C.: Human Rights Watch. Retrieved January 30, 2020 from: http://www.hrw.org/sites/default/files/reports/us0513_ForUpload_1.pdf.

Hunter Jr., J.A. \& Becker, J.V. (1994). The role of deviant sexual arousal in juvenile sexual offending: Etiology, evaluation, and treatment. Criminal Justice and Behavior, 21, 132-149.

Johnson, C.F. (2004). Child sexual abuse. The Lancet, 364, 462-470.

Jordan Institute for Families (2000). The effects of sexual abuse. Retrieved from http://practicenotes.org/vol5_no2/effects_of_sexual_abuse.htm 
Joyal, C.C., Cossette, A., \& Lapierre, A. (2015). What exactly is an unusual sexual fantasy? The Journal of Sexual Medicine, 12(2), 328-340.

Judge, A.M. (2012). “Sexting” among U.S. Adolescents: Psychological and legal perspectives. Harvard Review of Psychiatry, 20, 86-96.

Kahn, T.J. \& Chambers, H.J. (1991) Assessing re-offense risk with juvenile sexual offenders. Child Welfare, 70, 333-346.

Kaiser Family Foundation. (2020). State Health Facts: 2017. Retrieved from https://www.kff.org/other/state-indicator/children-byraceethnicity/?currentTimeframe $=1 \&$ selectedRows $=\% 7 \mathrm{~B} \% 22$ states $\% 22: \% 7 \mathrm{~B} \% 2$ 2oregon\%22:\%7B\%7D\%7D\%7D\&sortModel=\%7B\%22colId\%22:\%22Multiple $\% 20$ Races $\% 22, \% 22$ sort $\% 22: \% 22$ asc $\% 22 \% 7 \mathrm{D}$

Kaufman, K.L. (1994). Sexual History Form. Available from author. Columbus, OH: Children's Hospital.

Kaufman, K.L. (2014). Sexual History Form-Revised. Available from author. Portland, OR: Portland State University.

Kaufman, K.L. (1998). Sexual Fantasy Questionnaire. Available from author. Columbus, OH: Children's Hospital.

Kaufman K., Erooga M., Higgins D., Zatkin J. (2019) Youth-Serving Organization Safety Risks and the Situational Prevention Approach. In: Lonne B., Scott D., Higgins D., Herrenkohl T. (eds) Re-Visioning Public Health Approaches for Protecting Children. Child Maltreatment (Contemporary Issues in Research and Policy), vol 9. Springer, Cham. 
Kaufman, K.L., Hilliker, D.R., \& Daleiden, E.L. (1996). Subgroup differences in the modus operandi of adolescent sexual offenders. Child Maltreatment, 1, 17-24.

Kaufman, K.L., Holmberg, J.K., Orts, K.A., McCrady, F.E., Rotzien, A.L., Daleiden, E.L., \& Hilliker, D.R. (1998). Factors influencing sexual offenders' modus operandi: An examination of victim-offender relatedness and age. Child Maltreatment, 3, 349-361.

Kenny, D.T., Keogh, T., \& Seidler, K. (2001). Predictors of recidivism in Austrailian juvenile sexual offender treatment: Implications for treatment. Sexual Abuse: A Journal of Research and Treatment, 13, 131-148.

Klein, C.A. (2014). Digital and divergent: Sexual behaviors on the internet. The Journal of the American Academy of Psychiatry and the Law, 42(4), 495-503.

Kohlberg, L. (2008). The development of children's orientations toward moral order: 1. Sequence in the development of moral thought. Human Development, 51, 8-20.

Kuhn, D. (2009). Adolescent thinking. In R. Lerner \& L. Steinberg (Eds.), Handbook of adolescent psychology (3rd ed., pp. 151-185). New York: Wiley.

Langevin, R., Lang, R.A., \& Curnoe, S. (1998). The prevalence of sex offenders with deviant fantasies. Journal of Interpersonal Violence, 13, 315-327.

Larsen, E.N. (2013). Deviants or consenting adults: A human rights approach to defining and controlling deviant behavior. Sociology mind, 3(1), 1-6.

Laws, D.R. \& Marshall, W.L. (1990). A conditioning theory of the etiology and maintenance of deviany sexual preference and behavior. In W.L. Marshall, D.R. 
Laws, \& H.E. Barbaree (Eds), Handbook of sexual assault: Issues, theories, and treatment of the offender. New York: Plenum.

LeClerc, B., Proulx, J., \& Beauregard, E. (2009). Examining the modus operandi of sexual offenders against children and its practical implications. Aggression and Violent Behavior, 14, 5-12.

Leitenberg, H. \& Henning, K. (1995). Sexual fantasy. Psychological Bulletin, 117, 469496.

Letourneau, E.J. \& Caldwell, M.F. (2013). Expensive, harmful policies that don'twork or how juvenile sexual offending is addressed in the U.S. International Journal of Behavioral Consultation and Therapy, 8(3-4), 23-29.

Maniglio, R. (2011). The role of childhood trauma, psychological problems, and coping in the development of deviant sexual fantasies in sexual offenders. Clinical Psychology Review, 31(5), 748-756.

Mann, R.E., Hanson, R.K., \& Thornton, D. (2010). Assessing the risk for sexual recidivism: Some proposals on the nature of psychologically meaningful risk factors. Sexual Abuse: A Journal of Research and Treatment, 22(2), 191-217.

Marshall, W. L. (1979). Satiation therapy: A procedure for reducing deviant sexual arousal. Journal of Applied Behavior Analysis, 12, 377 - 389.

Marshall, W. L. \& Eccles, A. (1993). Pavlovian conditioning processes in adolescent sex offenders. In H.E. Barbaree, W.L. Marshall, \& S.M.Hudson (Eds), The juvenile sex offender. New York: Guilford. 
Marshall, W.L., Hudson, S.M., \& Hodkinson, , S. (1993). The importance of attachment bonds in the development of juvenile sexual offending. In H.E. Barbaree, W.L. Marshall, \& S.M. Hudson (Eds), The juvenile sex offender. New York: Guilford. Marshall, W.L. \& Marshall, L. (2000). The origins of sexual offending, Trauma, Violence, and Abuse: A Review Journal, 1, 250-263.

McCuish, E.C., Lussier, P., \& Corrado, R.R. (2015). Examining antisocial behavioral antecedents of juvenile sexual offenders and juvenile non-sexual offenders. Sexual Abuse: A Journal of Research and Treatment, 27(4), 414-438.

McMahon, P.M. \& Puett, R.C. (1999). Child sexual abuse as a public health issue: Recommendations of an expert panel. Sexual Abuse: A Journal of Research and Treatment, 11(4), 257-266.

Miranda, A.O. \& Corcoran, C.L. (2000). Comparison of perpetration characteristics between male juvenile and adult sexual offenders: Preliminary results. Sex Abuse, 12(3), 179-188.

McGuire, R.J., Carlisle, J.M., \& Young, B.G. (1965). Sexual deviations as conditioned behavior: A hypothesis. Behaviour Research and Therapy, 2, 185-190.

Miller, T.R., Cohen, M.A., \& Wiersema, B. (1996). Victim costs and consequences: A new look, Washington DC: U.S. Department of Justice, Office of Justice Programs.

Murray, L.K., Nguyen, A., \& Cohen, J.A. (2014). Child sexual abuse. Child Adolesc Psychiatr Clin N Am, 12(2), 321-337 
National Conference of State Legislatures. (2009). Legislation related to "Sexting" Retrieved from http://www.ncsl.org/?tabid=17756.

O’Keefe, G.S. \& Clarke-Pearson, K. (2011). Clinical report-the impact of social media on children, adolescents and their families. Pediatrics, 127, 800-804.

Oregon Youth Authority. (2016). OYA quick facts. Retrieved from https://www.oregon.gov/oya/docs/QuickFacts/QuickFacts-July2017.pdf

Oregon Youth Authority. (2020). OYA quick facts. Retrieved from https://www.oregon.gov/oya/docs/QuickFacts/QuickFacts.pdf

Oregon Youth Authority. (2019). Positive human development: At a glance. Retrieved from https://www.oregon.gov/oya/docs/glance/AtAGlance-PHD.pdf

Oregon Youth Atuhority. (no date). OYA facility services. Retrieved from http://www.oregon.gov/oya/Pages/facility_services.aspx\#Male_Offender_Faciliti es

Paolucci, E.O, Genuis, M.L., \& Violato, C. (2001). A meta-analysis of the published research on the effects of child sexual abuse. The Journal of Psychology, 135(1), 17-36.

Pinheiro, P.S. (2006). Report of the independent expert for the United Nations study on violence against children. available at: http://www.refworld.org/docid/43f30fab0.html [accessed March 5 2016]

Price, J., Patterson, R., Regnerus, M., \& Walley, J. (2016). How much more XXX is generation X consuming? Evidence of changing attitudes and behaviors related to pornography since 1973. Journal of Sex Research, 53(1), 12-20. 
Pullman, L. \& Seto, M.C. (2012). Assessment and treatment of adolescent sexual offenders: Implications of recent research on generalist versus specialist explanations. Child Abuse \& Neglect, 36, 203-209.

Ralph, N. (2016). A validation study of a prosocial reasoning intervention for juveniles under probation supervision. Sexual Offender Treatment, 11(2), 1-17.

Reynold, W.M. (1982). Development of reliable and valid short forms of the MarloweCrowne Social Desirability Scale. Journal of Clinical Psychology, 38(1), 119125.

Rich, P. (2006). Attachment and sexual offending: understanding and applying attachment theory to the treatment of juvenile sexual offenders. West Sussex, England: John Wiley \& Sons Ltd.

Rogojanski, J., Vettese, L. C., \& Anton, M.M. (2011). Coping with cigarette cravings: Comparison of suppression versus mindfulness-based strategies. Mindfulness, $2,14-26$.

Rokach, A. (1990). Content analysis of sexual fantasies of males and females. The Journal of psychology, 124(4), 427-436.

Rowan, E. L. (2006). Understanding child sexual abuse. Jackson: University of Mississippi Press.

Schaeffer, K. (2019). Most U.S. teens who use cellphones do it to pass time, connect with others, learn new things. Pew Research Center. Retrieved from https://www.pewresearch.org/fact-tank/2019/08/23/most-u-s-teens-who-usecellphones-do-it-to-pass-time-connect-with-others-learn-new-things/ 
Schneider, M., \& Hirsch, J. S. (2018). Comprehensive Sexuality Education as a Primary Prevention Strategy for Sexual Violence Perpetration. Trauma, Violence, \& Abuse. https://doi.org/10.1177/1524838018772855.

Schram, D.D., Malloy, C.D., \& Rowe, W.E. (1992, July). Juvenile sex offenders: A follow-up study of re-offense behavior. Interchange, 1-3.

Sedlak, A.J. and Broadhurst, D.D. (1996). Third National Incidence Study of Child Abuse and Neglect: Final Report. Washington, D.C.: U.S. Department of Health and Human Services.

Sedlak, A.J., Mettenburg, J., Basena, M., Petta, I., McPherson, K., Greene, A., and Li, S. (2010). Fourth National Incidence Study of Child Abuse and Neglect (NIS-4): Report to Congress, Executive Summary. Washington, DC: U.S. Department of Health and Human Services, Administration for Children and Families.

Seto, M.C. \& Lalumiere, M.L. (2010). What is so special about male adolescent sexual offending? A review of and test of explanations through meta-analysis. Psychological Bulletin, 136, 526-575.

Seto, M. C., Lalumière, M.L., \& Blanchard, R. (2000). The discriminative validity of a phallometric test for pedophilic interests among adolescent sex offenders against children. Psychological Assessment, 12, 319 - 327.

Seto, M. C., Murphy, W. D., Page, J., \& Ennis, L. (2003). Detecting anomalous sexual interests in juvenile sex offenders. Annals of the New York Academy of Sciences, $989,118-130$. 
Simon, D., Wurtele, S., \& Durham, R. (2008). Developmental experiences of child sexual abusers and rapists. Child Abuse and Neglect, 32, 549-560.

Snyder, H.N. (2000). Sexual assault of young children as reported to law enforcement: Victim, incident, and offender characteristics, Washington DC: U.S. Department of Justice, Office of Justice Programs.

Snyder, H., \& Sickmund, M. (1999). Juvenile offenders and victims: 1999 national report. Washington, DC: Office of Juvenile Justice and Delinquency Prevention.

Steinberg, L. (2004). Risk-taking in adolescence: What changes, and why? Annals of the New York Academy of Sciences, 1021, 51-58.

Steinberg, L., Albert, D., Cauffman, E., Banich, M., Graham, S., \& Woolard, J. (2008). Age differences in sensation seeking and impulsivity as indexed by behavior and self-report: Evidence for a dual systems model. Developmental Psychology, 44, $1764-1778$.

Steinberg, L., \& Monahan, K. (2007). Age differences in resistance to peer influence. Developmental Psychology, 43, 1531-1543.

Tabachnick, B.G. \& Fidell, L.S. (2007). Using multivariate statistics $\left(5^{\text {th }}\right.$ ed.). Boston, MA: Pearson Education, Inc.

Taylor-Browne, J. (1997). Obfuscating child sexual abuse II: Listening to survivors. Child Abuse Review, 6, 119-127.

Toft, S. \& Fellner, J. (2007). No easy answers: Sex offender laws in the US. (Vol. 19). Human Rights Watch. 
United States Department of Justice. (2015). Child pornography. Retrieved from https://www.justice.gov/criminal-ceos/child-pornography

U.S. Department of Health \& Human Services. (no date). Fourth national incidence study of child abuse and neglect (NIS-4): Report to congress. Washington DC: U.S. Department of Health and Human Services, Office of Population Affairs.

Veneziano, C.M. \& Veneziano, L. (2002). Adolescent sex offenders: A review of the literature. Trauma, Violence, \& Abuse, 3, 247-260.

Walsh, W., Wolak, J., \& Finkelhor, D. (2013). Sexting: When are state prosecutors deciding to prosecute? The third national juvenile online victimization study (NJOV-3). Crimes Against Children Research Center. Retrieved from http://www.unh.edu/ccrc/pdf/CV294_Walsh_Sexting\%20\&\%20prosecution_2-613.pdf

Washington Coalition of Sexual Assault Programs. (2016). Effects of child sexual abuse. Retrieved from http://www.wcsap.org/effects-child-sexual-abuse WHO. (2006). Preventing child maltreatment: A guide to taking action and generating evidence. World Health Organization.

Worling, J.R. \& Curwen, T. (2000). Adolescent sexual offender recidivism: Success of specialized treatment and implications for risk prediction. Child Abuse and Neglect, 24, 965-982.

Worling, J. (2012). The assessment and treatment of deviant sexual arousal with adolescents who offended sexually. Journal of Sexual Aggression, 18(1), 36-63 
Worling, J.R. \& Langstrom, N. (2006). Risk of sexual recidivism in adolescents who offend sexually: Correlates and assessment. In H.E. Barbaree, \& W.L. Marshall (Eds), The juvenile sex offender. New York: Guilford.

Wright, P.J. (2013). U.S. males and pornography, 1973-2010: Consumption, predictors, correlates. Journal of Sex Research, 50(1), 60-70.

Wurtele, S.K. (2009). Preventing sexual abuse of children in the twenty-first century: Preparing for challenges and opportunities. Journal of Child Sexual Abuse, 18, 118. 